\title{
Quantitative CT of the lungs: technical aspects and clinical studies
}

Citation for published version (APA):

Lamers, R. J. S. (1998). Quantitative CT of the lungs: technical aspects and clinical studies. [Doctoral Thesis, Maastricht University]. Maastricht University. https://doi.org/10.26481/dis.19981218rl

Document status and date:

Published: 01/01/1998

DOI:

10.26481/dis.19981218rl

Document Version:

Publisher's PDF, also known as Version of record

\section{Please check the document version of this publication:}

- A submitted manuscript is the version of the article upon submission and before peer-review. There can be important differences between the submitted version and the official published version of record.

People interested in the research are advised to contact the author for the final version of the publication, or visit the DOI to the publisher's website.

- The final author version and the galley proof are versions of the publication after peer review.

- The final published version features the final layout of the paper including the volume, issue and page numbers.

Link to publication

\footnotetext{
General rights rights.

- You may freely distribute the URL identifying the publication in the public portal. please follow below link for the End User Agreement:

www.umlib.nl/taverne-license

Take down policy

If you believe that this document breaches copyright please contact us at:

repository@maastrichtuniversity.nl

providing details and we will investigate your claim.
}

Copyright and moral rights for the publications made accessible in the public portal are retained by the authors and/or other copyright owners and it is a condition of accessing publications that users recognise and abide by the legal requirements associated with these

- Users may download and print one copy of any publication from the public portal for the purpose of private study or research.

- You may not further distribute the material or use it for any profit-making activity or commercial gain

If the publication is distributed under the terms of Article $25 \mathrm{fa}$ of the Dutch Copyright Act, indicated by the "Taverne" license above, 
Quantitative CT of the lungs 
(9) R.J.S. Lamers, Maastricht 1998

ISBN $909012277 \mathrm{x}$

Vormgeving en druk: Datawyse boekproducties Maastricht 


\section{Quantitative CT of the lungs}

Technical aspects and clinical studies

\section{PROEFSCHRIFT}

ter verkrijging van de graad van doctor aan de Universiteit Maastricht op gezag van de Rector Magnificus, Prof. Dr. A.C. Nieuwenhuijzen Kruseman volgens het besluit van het College van Decanen in het openbaar te verdedigen op vrijdag 18 december 1998 om 12.00 uur door R.J.S. Lamers 


\section{Promotores:}

Prof. Dr. J.M.A. van Engelshoven

Prof. Dr. E.F.M. Wouters

Co-promotor:

Dr. G.J. Kemerink

Beoordelingscommissie:

Prof. Dr. J.W. Arends (voorzitter)

Prof. Dr. C.L.A. van Herwaarden (Katholieke Universiteit Nijmegen)

Prof. Dr. S.C.M. Luijendijk

Prof. Dr. J.A. Verschakelen (Katholieke Universiteit Leuven)

The publication of this thesis was financially supported by:

Astra Pharmaceutica Benelux, GlaxoWellcome, Nycomed, Schering, Siemens, Zambon. 
Aan: mijn owders

Gertit

Michiel Jeroen, Stephan 



\section{Table of contents}

CHAPTER 1

General introduction 9

CHAPTER 2

Chronic obstructive pulmonary disease: a review 15

CHAPTER 3

CT lung densitometry: a review 29

CHAPTER 4

Scanner conformity in CT densitometry of the lungs 41

CHAPTER 5

On segmentation of lung parenchyma in quantitative computed tomography of the lung 51

CHAPTER 6

Reproducibility of spirometrically controlled CT lung densitometry in a clinical setting 69

CHAPTER 7

Chronic obstructive pulmonary disease: evaluation with spirometrically controlled CT lung densitometry 79

CHAPTER 8

Emphysema and airflow limitation in patients with advanced chronic obstructive pulmonary disease: a C.T study 93

CHAPTER 9

CT lung densitometry and visual assessment of thin-section $C \Gamma$ in the diagnosis of coal worker's pneumoconiosis 111 
CHAPTER 10

Summary and conclusions $\quad 127$

Samenvatting $\quad 133$

Acknowledgments 139

Curriculum vitae $\quad 141$ 


\section{General introduction}

Attenuation of an X-ray beam is caused by coherent scattering, the photo-electric effect and Compton scattering. The contribution of each these effects depends on the photon energy as well as the atomic numbers and the density of the exposed tissue. For the Xrays commonly used in computed tomography (CT), the photo-electric effect prevails for iodine or barium containing contrast media while the Compton effect is dominant for soft tissues. For bone both effects contribute significantly to the attenuation.

A CT scanner has the unique property that it accurately measures the X-ray attenuation in a cross-section of the body. The map of these attenuation values is the Cl image. However, it is common practice not to present the linear attenuation coefficient $(\mu)$ itself, but a derived quantity that is called the CT number. The definition is: CT number tissue $=1000\left(\mu_{\text {tissue }}-\mu_{\text {water }}\right) / \mu_{\text {water. }}$. Its unit is the Hounsfield or Hounsfield unit (HU). The CT image is generally evaluated by visual inspection, but the CT number can also be used in a quantitative way. Soon after the introduction of CT, applications were developed to quantify bone mineral content, diffuse lung pathology, and brain perfusion using inhalation of xenon. Diffuse lung pathology was quantified by the density of the lung, which has a linear relationship to the CT number.

CT densitometry of the lungs has been proposed as a sensitive tool to diagnose a wide variety of pulmonary disorders. Applications range from assessing pulmonary emphysema (1-14), sarcoidosis (15), panbronchiolitis (16), coall worker's pneumoconiosis (17), radiation induced pneumonitis (18), pulmonary edema (19), characterization of pulmonary nodules $(20)$, to bleomycin-related lung damage $(21,22)$.

Despite favorable reports $(4,5,12,13)$, CT densitometry of the lungs has not been yet applied as a clinical tool. Comparison of densitometric results obtained on different scanners and/or for different patients is difficult for various reasons. Most early CT scanners were not optimized for densitometry in the low density range. A large variability in absolute CT numbers between scanners of various manufacturers has been observed $(23,24)$. Moreover, internationally accepted protocols for CT lung densitometry were not available. CT numbers were affected by the reconstruction algorithm, slice thickness, object size and variations in the tube high voltage (24-29). Reproducibility errors due to the operator's intervention in the evaluation process may exceed $10 \mathrm{HU}$ (30). An additional problem in $\mathrm{CT}$ densitometry of the lungs (compared to bone and brain) is the influence of the levell of inspiration. At inspiration 
the lung contains more air compared to expiration which results in a considerable variation in lung density between in- and expiration (31). Patients will generally not be able to exhale to some fixed level. Control of the level of inspiration, especially when intermediate respiratory levels are required, is therefore mandatory to obtain reproducible results of lung density.

Modern $\mathrm{CT}$ scanners are much better optimized for densitometry in the low density range. The stability of our system for measuring air density is for example within 1 $\mathrm{HU}$ and for water density within $2 \mathrm{HU}$ (32). The accuracy and reproducibility for densities found in the lung are within 2-3 percent (32). Spirometric gating to control the respiratory level of the patient during data acquisition has increased the reproducibility of this method enormously (33), and so has semiautomated evaluation procedures that extract the relevant densitometric parameters from the CT data $(30,34)$.

Densitometry has the potential to diagnose and quantify pulmonary emphysema, chronic bronchitis, or interstitial lung disorders. Densitometry of the lungs can provide an objective quantification of changes in lung density over time and may determine at what level the airway obstruction may be occurring (35). Results may be useful for epidemiologic, transverse and longitudinal studies. It is not easy but neverthelless critical to distinguish chronic bronchitis from emphysematous patients with chronic obstructive lung disease because they have a different treatment and prognosis (36). In epidemiologic studies this distinction is important to discover risk factors. CT densitometry may be of help in lung reduction surgery in case of advanced emphysema by determining the site of greatest severity prior to operation that aims at reducing breathlessness (37). It may become possible to quantitatively assess the morphologic changes in the lungs after therapeutic interventions (38).

In spite of this large potential, CT densitometry of the lungs will not find broad introduction into clinical practice until all variables affecting densitometry are under control and scan protocols have been developed that give comparable results on all CT scanners.

\section{Outline of the thesis}

Chapter 2 focuses on several important aspects of chronic obstructive pulmonary disease: definition, pathologic characterization, clinical features, pulmonary function tests and radiological diagnosis. In chapter 3 the relevant literature on CT densitometry of the lung is reviewed. The technique of respiratory gating of the CT scanner, the technical parameters used in CT densitometry of the lung, as well as the method used in this thesis for extracting density information from CT scans are described. Subsequently, dosimetrical aspects of our CT densitometry protocol are discussed. In chapter 4 inter- and intrascanner conformity in CT densitometry of the lung is quantified. 
In chapter 5 the influence of scan protocol and post-processing on estimated lung density is evalluated as a contribution to the standardization of the technique. In chapter 6 the issue of reproducibility of spirometric gated CT lung densitometry at defined levels of inspiration is addressed. In chapter 7 the potential of quantitative CT to distinguish between patients with emphysema, chronic bronchitis and healthy individuals is studied. In chapter 8 we assessed the association of emphysema with airflow limitation in patients with chronic obstructive pulmonary disease and studied the point of debate whether the single breath carbon monoxide diffusing capacity can be used as an indicator for emphysema in these patients. In chapter 9 the usefulness of $\mathrm{CT}$ lung densitometry in interstitial lung disease is investigated and the most suitable level of inspiration to assess severity and extent of interstitial lung disease is defined in a population of coal miners. Chapter 10 contains a summary and conclusions of this thesis.

\section{REFERENCES}

1. Goddard PR, Nicholson EM, Laselo $G$, Watt I. Computed tomography in pulmonary emphysema. Clin Radiol 1982; 33:379 387 .

2. Hayhurst MD, Flenley DC, McLean A, et al. Diagnosis of emphysema by computerised tomography. Lancet 1984; 2:320-322.

3. Bergin $C$, Müller $N$, Nichols DM, et al. The diagnosis of emphysemat a computed tomographicpathologic correlation. Am Rev Respir Dis 1986; 133:541-546.

4. Gould GA, Macnee W, McLean A, et al. CT measurements of lung density in life can quantitate distal airspace enlargement: an essential defining feature of human emphysema. Am Rev Respir Dis 1988; 137:380-392.

5. Müller NL, Staples CA, Miller RR, Abboud RT. "Density mask": an objective method to quantitate: emphysema using computed tomography. Chest 1988; 94:782-787.

6. Biemacki W, Gould GA, Whyte KF. Flenley DC. Pulmonary hemodynamics, gas exchange, and the severity of emphysema as assessed by quantitative CT scan in chronic bronchitis and emphyserna. Am Rev Respir Dis 1989,139:1509-1515.

7. Kinsella M, Müller NL, Abboud RT, Morrison NI, DyBuncio A. Quantification of emphysema by computed tomography using a "density mask" program and correlation with pumonary function tests. Chest 1990; 97:3:15-321.

8. Knudson R], Standen IR, Kaltenborn WT, et al, Expiratory computed tomography for assessment of suspected pulmonary emphysema. Chest 1991;99:1357-1366.

9. Gould GA, Redpath AT, Ryan M, et al. Lung CT density correlates with measurements of airlow limitation and the diffusing capacity. Eur Respir J 1991;4:141-146.

10. Guenard $\mathrm{H}$, Mamadou $\mathrm{H}$, Diallo $\mathrm{HH}$, Laurent $F$, Vergere ). Lung density and lung mass in emphysema. Chest 1992; 102:198-203.

11. Gould GA, Redpath AT", Ryan M, et al. Parenchymal emphysema measured by CT hung density contelates with lung function in patients with bullous disease. Eur Respir $\int 1993 ; 6: 698-704$.

12. Gevenois PA, de Maertelaer $V$, De Vuyst $P$, Zanen J, Yernault JC. Comparison of computed density and macroscopic morphometry in pulmonary emphysema. Am I Respir Crit Care Med 1995: 152:653-657. 
13. Gevenois $\mathrm{PA}$, De Vuyst P, Sy $\mathrm{M}$, et al Pulmonary emphysema: quantitative CT during expiration. Radiology 1996:199:825-82\%.

14. Eda $\$$, Kubo K, Fujimoto K, Matsuzawa $Y$, Sekiguchi M, Sakai F. The relations between expiratory chest CT using helical CI and pumonary function tests in emphyserra. Am J Respir Crit Care Med $1997 ; 155: 1290 * 1294$.

15. Gilman MI, Laurens RG, Somogyi, Honig EG. CT attenuation walues of lung density in sarcoidosis. J Comput Assist Tomogr $1983 ; 7: 407-410$.

16. Murata $K, I$ toh $H$, Senda $M$, et all. Stratified impaiment of pulmonary ventilation in "diffuse panbronchiolitis:" PET and Cr studies. / Comput Assist Tomogr 1989; 13:48-53.

17. Bergin CI. Müller NL, Vedal S, Chan-Yeung M. CT in silicosis: correlation with plain films and pulmonary function tesis AJR 1986; 146:477-4833.

18. van Dyk $\mathrm{J}_{\mathrm{y}}$ Keane TJ, Rider WD. Lung density as measured by computerized tomography: implications for madiotherapy. Int J Radiol Oncol Biol Phys. 1982; 8:1363-1372.

19. Hedlund LW, Effmann E, Bates WM, Beck IW, Goulding PL, Putman CE. Pulmonary edema: a CT sudy of regional changes in lung density following oleic acid injury. J Comput Assist Tomogr 1982; 6:939-946.

20. Siegelman $\$ S$, Zerhouni $\mathbb{N} A_{0}$ Leo FP, Khour NF, Stitik FP. CT of the pulmonary solitary nodule. AJR $1980 ; 135: 1-13$.

21. Bellamy EA, Husband JE, Blaquiere RM, Law MR. Bleomycin-related lung damage: CT evidence. Radiology $1985 ; 156: 155-158$.

22. Bellamy EA, Nicholas D, Husband JE. Quantitative assessment of lung damage due to bleomycin us* ing computed tomography. Br J Radiol 1987; 60:1205-1209.

23. Levi C, Gray IE, McCullough EC, Hattery RR. The unreliability of CT numbers as absolute values. AlR $1982 ; 139: 4433-447$.

24. Zerhouni EA, Spivey JF, Morgan RH, Leo FP, Stitik FP, Siegelman SS. Factors influencing quantitative CT measurements of solitary pulmonary nodules. J Comput Assist Tomogr 1982; 6:1075-1087.

25. McCullough EC, Morin RL. CT-number variability in thoracic geonetry. AJR 183; 141:135-140.

26. Adams H, Bernard MS, McConnochie K. An appraisal of CT pulmonary density mapping in normal subjects. Clin Radiol 1991; 43:238-242.

27. Kemerink GJ, Kruize HH, Lamers RJS. Density resolution in quantitative computed tomography of foam and lung, Med Phys 1996; 23:1697-1708.

28. Kemerink G], Kruize HH, Lamers R]S, van Engelshoven MA. CT lung densitometry: dependence of CT number histograms on sample volume and consequences for scan protocol comparability. I Comput Assigt Tomogr 1997; 21:948-954.

29. Kemerink G], Kruize HH, Lamers RJS. The CT's sample volume as an approximate, instrumental measure for density resolution in densitometry of the lung. Med Phys 1997; 24:1615-1620.

30. Kalender WA, Fichte H, Bautz W, Skalej M. Semiautomatic exaluation procedures for quantitative CT of the lung. I Compun Assist Tomogr 1991; 15:248-255.

31. Robinson PI, Kreel L. Pulmonary tissue attenuation with computed tomography: comparison of in. spiration and expiration sans. . Comput Assist Tomogr 1979; 3:740-74:8.

32. Kemerink GJ, Lamers RJS, Thelissen GRP, van Engelshoven JMA. CT densitometry of the lungs: scanner performance. J Comput Assist Tomogr 1996; 20:24-33.

33. Kalender WA, Rienmuller R, Seissler W, Behr I, Welke M, Fichte H. Measurement of pulmonary parenchymal attemsation: use of spirometric gating with quantitative CT. Radiology 1990; 175:265-268.

34. Archer DC, Coblentz CL, deKemp RA, Nahmias C, Norman G. Automated in vivo quantification of emphysema. Radiology 1993; 188:835-838.

35. Müller NL, Thurlbeck WM. Thin-section CT, emphysema, air trapping, and airway obstruction. Radiology $1996 ; 199: 621-622$

36. Burrows $B$ Bloom WW, Traver GA, Cline MG. The course and prognosis of different forms of chronic airways obstruction in a sample from the general population. N Engl I Med 1987; 317:1309-1314. 
37. Bae KT, Slone RM, Gierada DS, Yusen RD, Cooper DD. Patients with emphysema: quantitative CT analysis before and after lung volume reduction surgery. Radiology 1997; 203:705-714.

38. Holbert MM, Brown ML, Sciurba FC, Keenan RI, Landrenea RI. Holzer AD. Changes in lung volume and volume of emphysema after unilateral lung reduction surgery: analysis with CT lung densitometry. Radiology 1996; $201: 793-797$. 

CHAPTER 2

Chronic obstructive pulmonary disease: a review 


\subsection{Introduction}

Chronic obstructive pulmonary disease (COPD), a relatively common form of chronic lung disease, is defined as a disease state characterized by the presence of airflow obstruction due to emphysema or chronic bronchitis (1). The airflow obstruction is generally progressive. The major risk factor is cigarette smoking and $15 \%$ of all cigarette smokers develop clinically significant COPD (2). Knowledge of the prevalence of COPD in the Netherlands is incomplete. In the United States it is estimated that about 4-6\% of adult white males and about 1-3\% of adult white females suffer from COPD (1). In 1991, in the United States, the associated death rate was 18.6 per 100.000 persons per year. Age of starting, total pack-years and current smoking status are predictive of COPD mortality. COPD ranked as the fourth leading cause of death in the United States (1). It is essential to distinguish emphysematous from chronic bronchitis forms of COPD since they have such a different prognosis (3).

\subsection{Definitions}

Emphysema is a pathological entity defined by the American Thoracic Society in strictly morphologic terms as a condition of the lung characterized by abnormal permanent enlargement of airspaces distal to the terminal bronchioles accompanied by destruction of their walls and without obvious fibrosis (4).

Chronic bronchitis is a poorly defined condition. In contrast to emphysema, chronic bronchitis is defined on clinical criteria as recurrent excess of secretion in the bronchial tree occurring on most days for at least three months of the year for at least two successive years in a patient in whom other causes of cough have been excluded (5). Although not included in the definition of chronic bronchitis, chronic airflow obstruction is commonly present.

\subsection{Pathologic characterization of COPD}

Enphysema is classified by the way the acinus is involved $(1,6,7)$. Three patterns are recognized, each having a characteristic distribution within the lung. The most common type is proximal acinar emphysema which characteristically destroys the proximal portion of the acinus with a predominance in the upper zones of the lungs. It is the most frequent type of emphysema among cigarette smokers $(6,8)$. The second type of emphysema, panacinar emphysema, destroys the acinus more or less uniformly. It is more common in the lower zones of the lungs, and is the predominant type of emphysema in patients with $\alpha 1$ - antitrypsin deficiency. The third type of emphysema, distal 
acinar emphysema, dominantly involves the peripheral part of the acinus. Distal acinar emphysema is almost always seen in the periphery of the lung, along interlobular fibrous septa or beneath the pleura. The remainder of the lung is often spared. Proximal acinar emphysema and panacinar emphysema may coexist (8).

Pathologic findings in the bronchial walls of patients with chronic bronchitis are a chronic inflammatory reaction in the small airways - airways less than $2-3 \mathrm{~mm}$ in diameter - consisting of cellular infiltration, goblet metaplasia, smooth muscle hyperplasia, accompanied by connecting tissue deposition $(9,10)$. Mucus secretion and mucus gland hyperplasia are characteristic histological findings in the large airways (11).

\subsection{Clinical features of COPD}

Patients with COPD have usually been smoking more than 20 cigarettes per day for more than 20 years before symptoms develop (1). Symptoms commonly present in the fifth decade of life with productive cough or an acute chest illness (1). Sputum production is insidious, initially occurring in the moming. Sputum is usually mucoid but becomes purulent with exacerbation. With progression, the intervals between acute exacerbation grow shorter. Dyspnea on effort usually does not occur until the sixth or seventh decade. It is clinically difficult to distinguish emphysema from chronic bronchitis because of the similar symptoms of wheezing, cough and shortness of breath $(12,13)$.

\subsection{Pulmonary function tests in COPD}

A decrease in maximal expiratory flow is a functional hallmark of COPD. Abnormalities in the small airways function tests are related to the inflammatory process in these small airways (14-17), the most important site of increased resistance in the lungs of patients with established COPD (18-21). The mechanisms by which the inflammatory changes in the airways lead to excessive airway narrowing remains unclear. Forced expiratory volume in one second $\left(\mathrm{FEV}_{1}\right)$ is the most useful test of airflow dynamics. In this thesis, patients with fixed airway obstruction were considered to have COPD if the prebronchodilator $\mathrm{FEV}_{1}$ was less than $70 \%$ of the predicted value, if this $\mathrm{FEV}_{1}$ did not change over several months and if a difference between prebronchodilator and postbronchodilator values of FEV 1 did not exceed $10 \%$ of predicted.

Airflow limitation at spirometry and disturbance of gas exchange, manifested by reduced single-breath carbon monoxide diffusing capacity ( $D L_{C O}$ ), have been proposed as the most accurate and specific pulmonary function tests for pulmonary em- 
physema (22). Correlations between various pulmonary function tests and the pathologic extent of emphysema range from 0.4 to $0.7(23)$. The mechanism of airflow limitation in emphysema involves emphysematous destruction of parenchymal support of the peripheral airways and/or loss of elastic recoil force driving air out of the lung $(24,25)$. Although emphysema is considered part of the spectrum of chronic obstructive pulmonary diseases, expiratory air-flow obstruction is not invariable present (26). Patients with severe emphysema may have few symptoms and little evidence of airflow obstruction, while patients with moderate grades of emphysema may have significant disabling pulmonary dysfunction (27). Patients may have up to 30 percent of their lung function involved with emphysema and no evidence of functional impairment (28). Many authors agree that emphysema has a dominant role in causing airflow limitation $(25,26,28)$, whereas others state the opposite $(2,30,31)$.

The $\mathrm{DL}_{\mathrm{CO}}$, whether measured using a single breath or a steady state method, expressed in absolute terms, as percent predicted, or as function of the alveolar volume, has constantly been the best predictor of emphysema $(22,32)$. The impairment of DL $L_{C O}$ present in emphysema may arise from breakdown of alveolo-capillary surface area, the concomitant loss of pulmonary arteries leading to impairment in diffusion in the enlarged airspaces and decreased diffusion of carbon monoxide into the pulmonary blood. Patients are considered to have emphysema if they meet the ATS criteria for functional emphysema: $\mathrm{DL}_{\mathrm{CO}}$ less than $80 \%$ of the predicted value and $F E V_{1}$ less than $80 \%$ of the predicted value (4). However, $\mathrm{DL}_{\mathrm{CO}}$ is decreased in a number of pulmonary disorders (33). DL $L_{C O}$ provides only a very imprecise quantification of the grade of macroscopic emphysema (26). The upper zones of the lungs are a relatively silent region where extensive destruction may occur before functional abnormalities become evident (34). Therefore there may be an apparent discrepancy between the radiologic extent of emphysema and the physiologic measurements of emphysema.

\subsection{Radiologic diagnosis of COPD}

\section{Chest radiography}

Findings indicative of emphysema on a chest radiograph are overinflation and pulmonary vascular abnormalities $(13,28)$. Overinflation is represented by a low and flat diaphragm and an enlarged retrosternal air-space. Pulmonary vascular abnormalities are indicated by excessive rapid tapering of vascular shadows, local areas of oligemia and prominent hilar vascular shadows (13). However, these findings are difficult to detect before the disease is severe (6). The diagnosis of emphysema from the chest radiograph is neither very accurate nor specific (35). There are no criteria which permit more than an approximate estimate of the severity of emphysema from the chest ra- 
diograph. The chest radiograph diagnosis is usually "yes-no" which is in contradiction with the natural behavior of the disease which is slowly progressive and of insidious onset. It is thus hardly possible to quantitate the severity and extent of emphysema by chest radiography. Chest radiographs are useful for making a diagnosis of moderate to severe emphysema.

Radiographic abnormalities in patients with chronic bronchitis generally reflect bronchial wall thickening (36). However, bronchial wall thickening has no fixed defnitions and abnormalities are non-specific. The diagnosis of bronchial wall thickening is very subjective and is influenced by the degree of inspiration. Up to $50 \%$ of patients with clinically defined chronic bronchitis have normal chest film (37).

\section{Computed tomography}

It is generally conceded that CT is superior to chest radiography in assessing the presence and extent of parenchymal abnormalities $(23,38)$. Emphysema is diagnosed on CT by hypodense areas of pulmonary parenchyma without clear margins, usually associated with pruning, obliteration, ox abnormal course of the adjacent pulmonary vessels. In the past CT scans were obtained with long scan times and $1-\mathrm{cm}$ collimation. However, thick sections resulted in volume averaging within the plane of section and a decreased resolution so that the anatomic detail provided was insufficient. Modern CT scanners provide $1-\mathrm{mm}$ collimation and scan times of 1 second with high spatial resolution, enabling recognition of normal and diseased lobular structures. With optimal technical parameters, namely large matrix size, small field of view, thin-sections, and use of an ultra-high resolution filter, spatial resolution is reported to be 0.5 to 0.8 $\mathrm{mm}$ (39). High-resolution CT (HRCT) has shown to be superior to conventional CT. in evaluating chronic diffuse lung disease (23), and the overall extent of emphysema is more conspicuous and more reliably diagnosed (40). HRCT is recognized as a power. ful technique for the identification of the type of emphysema (41). Proximal emphysema of mild to moderate severity appears as a localized area of abnormally low attenuation located near the vessels in the center of a secondary lobule, approximately $0.5 \mathrm{~cm}$ in diameter (Figure 1). Panacinar emphysema is characterized by extensive ar eas of uniform low attenuation and a predominant lower lobe distribution (Figure 2). Distal acinar emphysema may be recognized by bullae, with a tendency to subpleural localizations (Figure 3). As emphysema becomes more severe, classification into subtypes becomes difficult. Different types of emphysema may coexist in the same person. A disadvantage of HRCT is that it is not so good at showing the vascular changes (42).

Specific HRCT findings of chronic bronchitis are usually lacking. Possible manifestations of chronic bronchitis on HRCT are thickening of the bronchial wall and some small airway abnormalities. A nodular branching structure resembling a bud- 


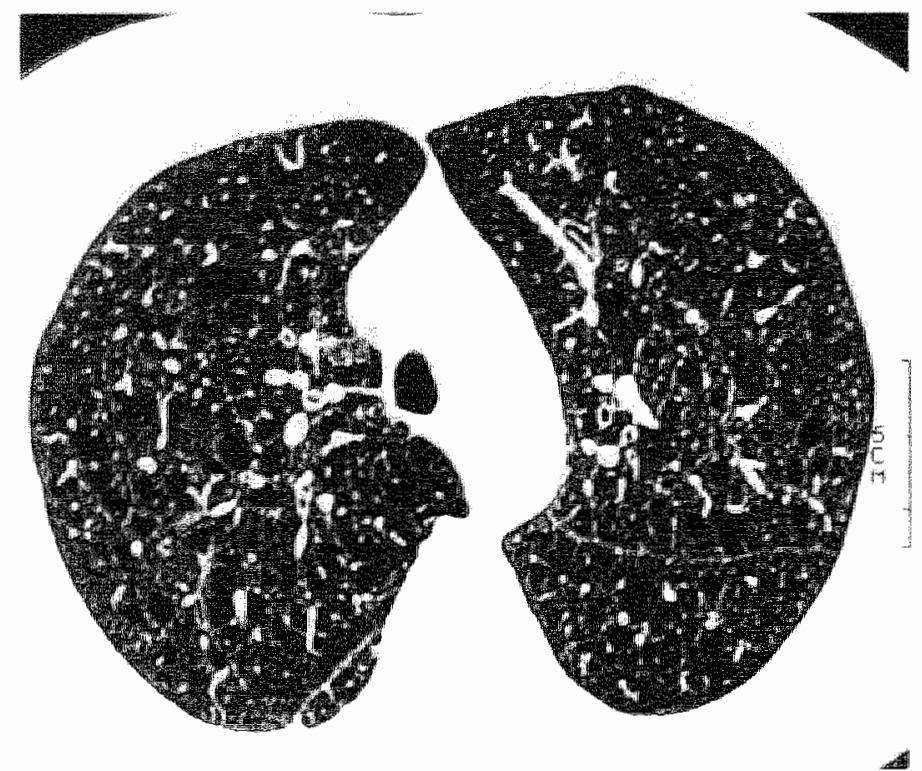

Figure 1. Proximal acinar emphysema: HRCT scan through the upper zones of the lungs shows multiple localized areas of low attenuation, $2 \% 10 \mathrm{~mm}$ in diameter.

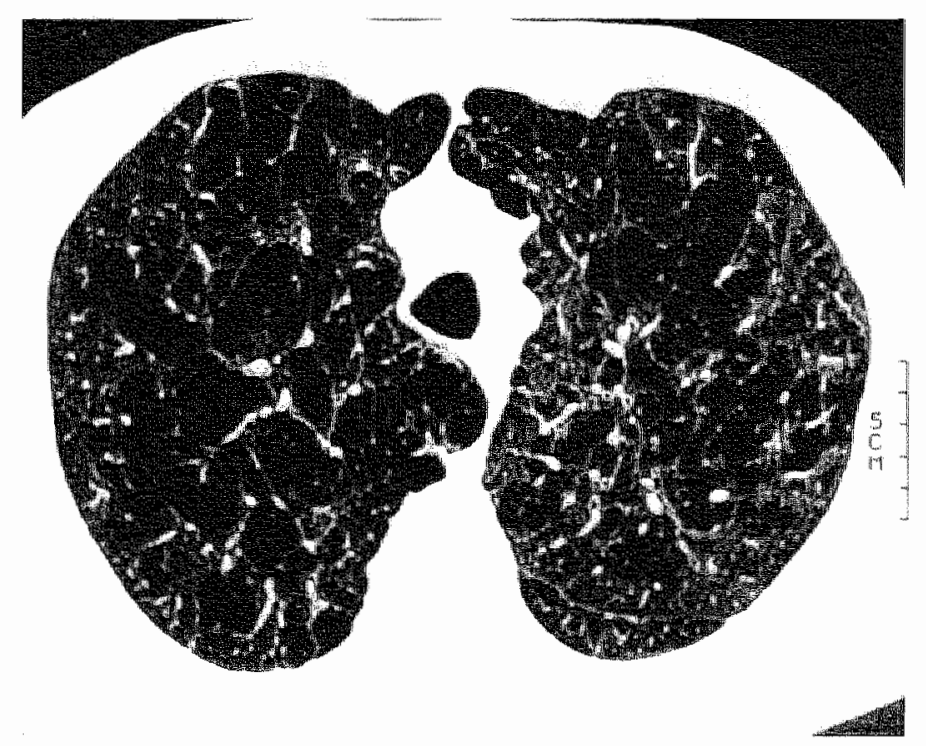

Figure 2. Advanced panacinar emplysema: HRCT scan through the upper zones of the lungs demonstrates widespread areas of low attenuation and dimuished vascular markings. 


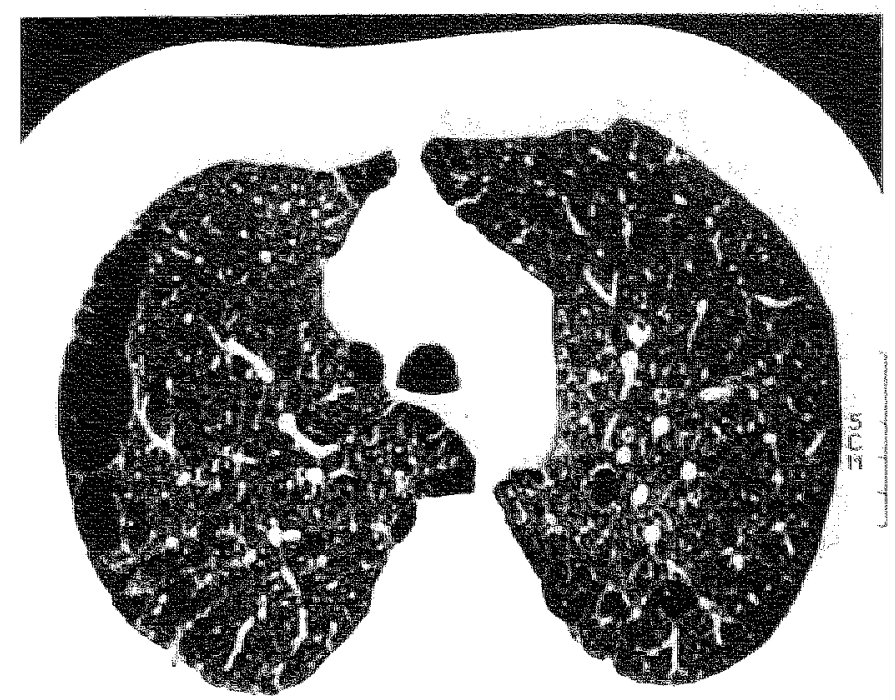

Figure 3. Distal acinar emphysema: HRCT scan through the upper zones of the lungs shows areas of subpleural emphysema most appropriately termed bullae.

ding tree in the lung periphery representing bronchiolar dilatation and filling of the lumen by mucus, pus, or fluid may be observed (42). At full inspiration, intervening lung parenchyma is normal. Expiratory CT scans may be useful to identify the subtle airway abnormalities indicative of air trapping in the evaluation of COPD (43).

\subsection{Quantification of emphysema on CT}

There are two ways to quantify the extent of emphysema using CT; subjectively by visual inspection for areas of emphysematous destruction, and objectively, by quantitative analysis of the density of the lung.

\section{Visual assessment of emphysema}

CT compares favorably to pathological specimens and may be considered the gold standard for diagnosing emphysema in vivo $(40,44-47)$. Emphysema is identified as non-peripheral unmarginated low-attenuation areas usually associated with vascular pruning or pulmonary vascular distortion. Three visual scoring methods have been employed to estimate the degree of emphysema: a grid method, a panel grading method, and a direct observational method introduced by Goddard. 
The grid method is an accurate method of assessing emphysema on CT according to a pathological grid scoring system $(45,46)$. A grid with squares corresponding to $1 \mathrm{~cm}^{2}$ is placed on the CT image and the extent and severity of emphysema in each square is determined. Approximately 50 boxes per lung field, and 500 boxes per patient have to be analyzed. The CT emphysema score is expressed in the percentage of squares containing emphysema. The grid method is complex and extremely time-consuming and therefore not suitable for clinical application.

The panel grading scoring method $(40,46)$ is a modification of the application of a panel of 16 standard photographs of parasagittal whole lung sections established by Thurlbeck et al (48). The grading panel of standards is based on recurring patterns of emphysematous lung destruction. Five CT slices are graded separately on a scale of zero to 100 by comparing the destructive changes seen radiographically to the grading panel according to the percentage that demonstrated changes suggestive of emphysema. Mild, moderate, and severe emphysema are scored 20,50 and 80 , respectively. The most severely emphysematous lung is given a score of 100 . The standards in this system are allocated at intervals of 5 from zero to 50 and at intervals of 10 from 60 to 100. The mean of CT scores for the CT slices is calculated. Comparison of horizontal CT slices against the parasagittal sections of the panel is observer dependent.

The method introduced by Goddard et al is a direct observational method of assessing the extent of emphysema (47). The left and right lung in each CT slice are graded separately according to the percentage area that demonstrates changes suggestive of emphysema. A grade of 0 is given if there was no abnormality. If less than $25 \%$ of the pulmonary parenchyma in a slice is considered to show vascular disruption and low attenuation compared with the remaining lung parenchyma, the score is 1 ; between $25-50 \%$, the score is 2 ; between $50-75 \%$, the score is 3 ; up to a maximum of 4 if more than $75 \%$ of the lung parenchyma on one side shows changes suggestive of emphysema. The maximal emphysema score per CT slice is 8. Approximately 16-24 CT slices have to be evaluated. The CT emphysema score of each patient is calculated by adding the emphysema score for each level and dividing by the total possible maximal score for each individual.

In this thesis, assessment of emphysema was carried out to a visual score introduced by Sakai et al (49) which is in fact a modification of the method of Goddard (47). This method, contrary to the method of Goddard, also takes the severity of emphysema into account. It is a very useful method for application in clinical practice. Assessing severity and extent of pulmonary emphysema according to this method takes approximately three minutes per patient. The interobserver correlation is high $(r=0.89$, $p<0.001$ ). Grading emphysema on CT using this method is as precise as using the grid method (49). Each section of the lung is scored for the severity according to a fourpoint scale, as follows: 0 , no emphysema; 1, low-attenuation areas smaller than $5 \mathrm{~mm}$ 
in diameter; 2, circumscribed low-attenuation areas larger than $5 \mathrm{~mm}$ in diameter in addition to those smaller than $5 \mathrm{~mm}$ in diameter; and 3, diffuse low-attenuation areas without intervening normal lung of large, confluent low-attenuation areas. The extent of emphysema was scored for each section also according to a four point scale; 1 , less than $25 \%$ cross-sectional area involvement; $2,25 \%-50 \%$ cross-sectional involvement; $3,50 \%-75 \%$ cross-sectional area involvement; and 4 , more than $75 \%$ cross-sectional area involvement. The CT emphysema score is based on the evaluation of $5 \mathrm{CT}$ sections. For each of the 10 lung sections, the score for severity of emphysema was multiplied by the score for the extent, and the resultant scores were subsequently summed to a final CT emphysema score which could range from zero to 120 . The scoring method of Sakai is illustrated in the figures 4 to 6.

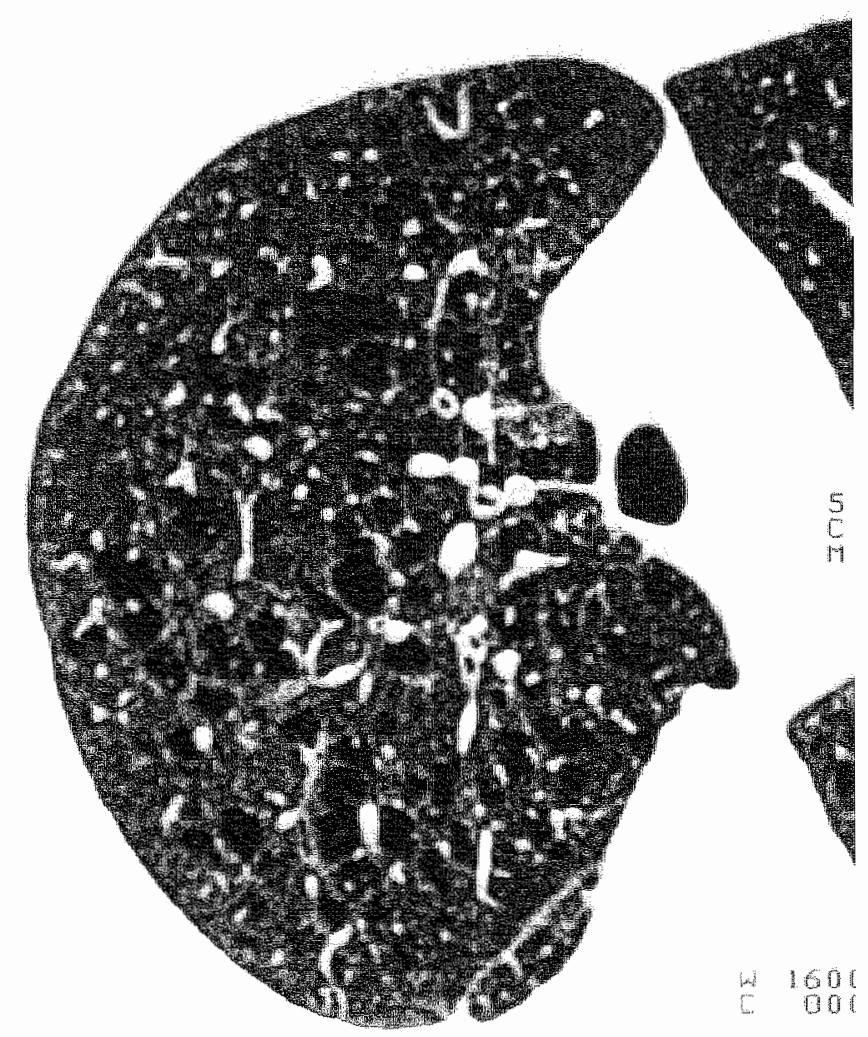

Figure 4. MRCT scan of the right lung denonstrates proximail acina umphysema and normal wascular markings with a severify scone of 1 and an ewent score of 4 . 


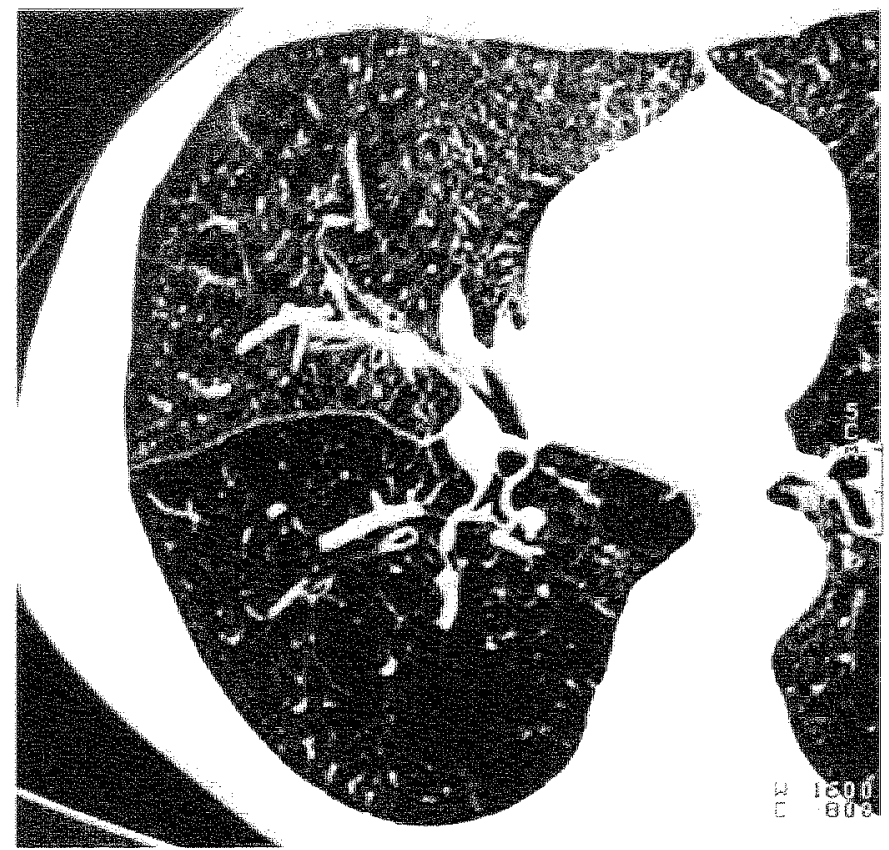

Iigure 5. HRCI scan of the right lung shows panacinar emphysema and diminished vascular markings with a sewerity score of 3 and an extent score of 2 .
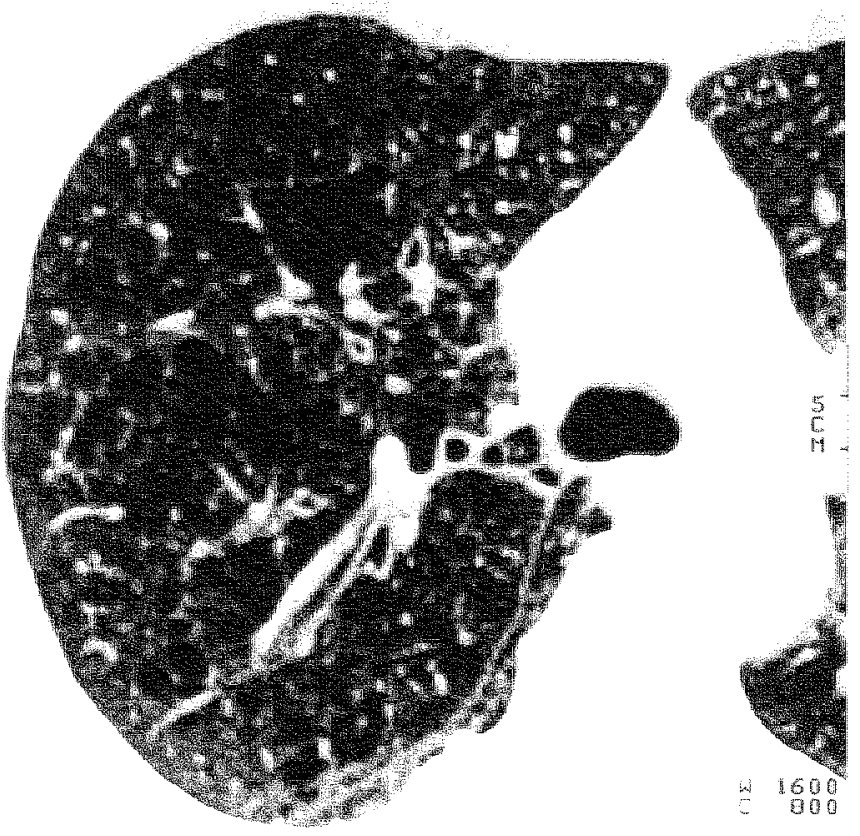

Figure 6. HRCT scan of the right lung shows panacinar emphysema and normal vascaliar markings with a severity score of 2 and an extent score of 4 . 


\section{Quantitative assessment of emplysema}

Drawbacks of visual scoring techniques relate to the experience of the observer and the effort and time they require. Ultimate results are dependent on window settings. There is a 10\% intra- and interobserver variability (50). The mildest forms of emphy. sema may be missed (45). To avoid subjectivity, methods have been developed to calculate objectively the relevant density characteristics from the CT sections. The value of CT densitometry in the assessment of parenchymal lung disease is discussed in chapter 3.

\subsection{Correlation between visual assessment of emphysema by CT and pathologic grading}

Foster et al (50) tried to establish. which CT sign is most valuable for the diagnosis of emphysema on CT. They evaluated the in vivo CT scans of 25 patients who later died. CT scans were obtained with $1-\mathrm{cm}$ collimation at $1-\mathrm{cm}$ intervals through the chest. The following signs were evaluated: nonperipheral low-attenuation areas, pulmonary vascular pruning, pulmonary vascular distortion, and visual density gradient. Postmortem the lungs were fixed in the inflated state and assessed for the presence and severity of proximal acinar emphysema. Nonperipheral low-attenuation areas formed the CT sign which correlated best with the pathologic grade of emphysema (upper zones: $r=0.84$, lower zones: $r=0.78$ ).

Bergin et al (44) assessed the accuracy of $C T$ in the diagnosis of emphysema. One$\mathrm{cm}$ collimated scans were obtained at $1-\mathrm{cm}$ intervals from 32 patients prior to surgery for removal of suspected tumors. The extent of emphysema seen on CT was assessed using the method of Goddard (48). They found significant correlations between the CT score and the pathologic score for resected lobes $(r=0.57, \mathrm{p}<0.001)$ and for $\mathrm{re}-$ sected lungs $(r=0.64, p<0.001)$. The authors concluded that $C T$ was a useful adjunct in the assessment of severity and extent of emphysema.

Miller et al (45) analyzed $1-\mathrm{cm}$ and $1.5-\mathrm{mm}$ collimated scans obtained at $1-\mathrm{cm}$ intervals from 38 patients, who underwent lobe or lung resection for malignancy, for the purpose of CT-pathologic correlation. The CT emphysema score was assessed using a grid method whereas the pathologic grade of emphysema was assessed using the grid method as well as the panel grading method. Correlations were better for the panel grading method than for the grid method. Correlation between the CT emphysema score and the pathologic grade was 0.81 for the $1-\mathrm{cm}$ collimated and 0.85 for the $1.5-\mathrm{mm}$ collimated scans. CT underestimated the extent of proximal acinar emphysema as well as panacinar emphysema because most lesions less than $0.5 \mathrm{~cm}$ were missed. 
Hruban et al (46) assessed the accuracy of HRCT in the diagnosis of emphysema. Autopsied lungs were dried and fixed for the purpose of the radiologic study. Five 2-mm collimated scans were obtained of the lung specimens. Subsequently the pathologist sectioned the lung specimens along the planes of the CT scans. The extent of emphysema seen on CT was assessed using the panel-grading method for the purpose of $\mathrm{CT}$ - pathologic correlation. The authors found significant correlation between the $\mathrm{CT}$ emphysema score and the pathologic grade $(\mathrm{r}=0.91, \mathrm{p}<0.005)$. They considered visual assessment of emphysema on HRCT, a reliable technique for the detection and quantification of emphysema even in its earliest stages.

Kuwano et al (40) determined the accuracy of CT scans in the assessment of clinically silent and mild emphysema. One- and 5-mm collimated scans. were obtained from 42 patients who underwent surgery for removal of a pulmonary nodule. The extent of emphysema seen on CT was assessed using the panel grading method. The correlations between the CT emphysema score and the pathological grade were 0.68 for the $1-\mathrm{mm}$ collimated scans and 0.75 for the $5-\mathrm{mm}$ collimated scans. The authors concluded that HRCT scans can help to identify the presence and grading of mild emphysema. The detection of areas of low attenuation was more important in the diagnosis of emphysema than the evaluation of the vascular pattern.

\section{References}

1. Standards for the diagnosis and care of patients with chronic obstructive pulmonary disease. Am I Respir Crit Care Med 1995; 152:577-\$120.

2. Hogg JC, Wright JL, Wiggs BR, Coxson HO, Opazo Saez A, Paré PD. Lung structure and function in cigararette smokers. Thorax 1994; 49:473-478.

3. Burrows B, Bloon IW, Traver $G A_{\text {, }}$ et al. The course and prognosis of different forms of chronic airways obstruction in a sample from the general population. N Engl J Med 1987; 317:1309-1314.

4. Snider GL, Kleinerman J, Thurbeck WM, Rengali ZK. The definition of emphysema: report of $\mathrm{Na-}$ tional Hearl, Lung, and Blood Institute, Division of Lung Diseases Workshop. Am Rev Respir Dis $1085 ; 132: 182-185$

5. American Thoracic Society. Chronic bronchitis, asthma, and pumonary emphysema: Statement by the Committee on Diagnostic Standards for Nontuberculous Respiratory Diseases. Am Rev Respir Dis $1962 ; 857762-768$.

6. Snider GL. Emplysema: the first two centuries and beyond. Am Rev Respir Dis 1992; 146:1334-1344.

7. Stem EI, Frank MS. CT of the lung in patients with pulmonary emphysema: diagnosis, quantification, and correlation with pathologic and physiologic findings. AJR 1994; 162:791-798.

8. Thurlbeck WM. Pathology of the lung. New York: Thieme Medical Publishers, 1987. 538-550.

9. Mullen JMB, Wright JL, Whggs BR. Reassessment of airways in chronic bronchitis. Br Med /1985; $291: 1235-1239$.

10. American Thoracic Society. Standards for the diagnosis and care of patients with chronic obstructive pulmonary disease (COPD) and astlama. Am Rew Respir Dis 1987; 136:225 243.

11. Reid L. The pathology of emphysema. London: Loyd-Duke Ltd., 1967.

12. Snider GL. Distinguishing among asthma, chronic bronchitis, and emphysema. Chest 1985; 87:355-39S.

13. Sanders C. The radiographic diagnosis of emphysema. Radiol Clin North An 1991; 29:1019-1030. 
14. Wright IL, Lawson LM, Pare PD, Kennedy S, Wiggs B. Hogg IC. The detection of mall airways disease. Am Rev Respir Dis 1984; 129:989-994.

15. Cosio $\mathrm{M}$, Ghez $2 \mathrm{H}$, Hogg $/ \mathrm{C}$, et al. The relations between structural changes in small airways and pulmonary function tests. N Engl J Med 1978; 298:1277-1281.

16. Cosio MG, Hale KA, Niewoehner DE. Morphologic and morphometric effects of prolonged cigarette smoking on the small airways. Am Rew Respir Dis 1980; 122:265-271.

17. Berend N, Wright IL, Thurbeck WM, Marlin GE, Woolcock A). Small airways disease: reproducibility of masurements and correlation with lung function. Chest 1981; 79:263-268.

18. Hogg IC, Macklem PT, Thurlbeck WM. Site and nature of airway obstruction in chronic obstructiwe. lung disease. N Engl I Med 1968; 278:1355-1360.

19. Thurlbeck WM, Henderson IA, Fraser RG, Bates DV. Chronic obstructive lung disease: a comparison between clinical, roentgenologic, functional, and morphologic criteria in chronic bronchitis, emphysema, asthma, and bronchiectasis. Medicine 1970; 49:81-145.

20. Yana M, Sekizawa K, Ohrui T, Sasaki H, "Takishima T. Site of airway obstruction in pulmonary disease: direct measurement of intrabronchial pressure. I Appl Physiol 1992; 72:1016-1023.

21. Petty TL, Silvers GW, Stanford RE. Mild emphysema is associated with reduced elastic recoil and increased lung size but not with airflow limitation. Am Rev Respir Dis 1987; 136:867-871.

22. Gelb AF, Gold WM, Wright R, Bruch HR, Nadel IA. Physiologic diagnosis of subclinical emphysetria. Am Rev Respir Dis 1973; 107:50-64.

23. Müller NL. Clinical value of high-resolution CT in chronic diffuse lung disease. AJR 1991; 157:1163. 1170.

24. Butler $\mathrm{J}$, Caro $\mathrm{C}$, Alkaler $\mathbb{R}_{\text {, }} \mathrm{Dubois} \mathrm{B}$. Physiologic factors affecting airway resistance in nomal subjects and in patients with obstructive anirways disease. I Clin Invest 1960; 39:584-591.

25. Demedts $M$, Aumann J. Early emphysema: ten years" evolution. Chest 1988; 94:337-342.

26. Nagai A, West WW, Thurlbeck WH. The National Institutes of Health Intermittent Positive Pressure Breathing Trial; pathology studies: IT. Correlation between morphologic findings, clinical findings, and evidence of expiratory airflow obstruction. Am Rev Respir Dis 1985; 132:946-953.

27. Groskin SA. Emphysema: fact, fiction, or just a lot of hot air. Radiology 1992; 183:319m,320.

28. Pratt PC. Role of conventional chest radiography in diagnosis and exclusion of emphysema. Am I Med 1987; 82:998 1006.

29. Miniati $M$, Filippi E, Falaschi $F$, et al. Radiologic evaluation of emphysema in patients with chronic obstructive pulmonary disedse. Am Rev Respir Crit Care Med 1995; 151:1359-1367.

30. Gelb $A F$, Schein $M, K u e i$, el al. Limited contribution of enphysema in advanced chronic obstructive pulmonary disease. Am Rex Respir Dis 1993; 147:1157-1161.

31. Gelb AF, Hogg JC, Müllew NL, et all. Contribution of emphysema and small airways in COPD. Chest 1996; 109:353-359.

32. Thurlbeck WM. Overwiew of the pathology of pulmonary emphysema in the human, Clin Chest Med. $1983 ; 4: 337-350$.

33. Klein JS, Gamsu G, Webb RW, Golden JA, Müller NL. High-resolution CT diagnosis of emphysema in symptomatic patients with nomal chest radiographs and isolated low diffusing capacity. Radiology $1992 ; 182.817-821$.

34. Gurney IW, Jones KK, Robbins RA, et al. Regional distribution of emphysema: correlation of highresolution CT with pulmonary function tests in unselected smo-kers. Radiology 1992; 183:457-463.

35. Burki NK. Roentgenologic diagnosis of emphysema: accurate or not? Chest 1989; 95:1178-1179.

36. Fraser RG, Fraser RS, Renner IW, Bernard C. Fitzgerald PI. The roengenologic diagnosis of dronic bronchitis: a reassessment with emphasis on parahilar bronchi seen end -on. Radiology 1976; 120:1-9.

37. Simon $G$. Chronic bronchitis and emphysema: a symposium. III. Radiologic changes in chronic bronchitis. Br J Radiol 1959: 32:292-294.

38. Sanders $\mathrm{C}$, Nath PH, Bailey WC. Detection of emphysema with computed tomography: correlation with pumonary function tests and chest radiography. Invest Radiol 1988;23:262-266. 
39. Naidich DP, Zerhouni EA, Siegelman SS. Computed tomography of the thorax. New York: Raven Press, 1984 .

40. Mulller NL. CT diagnosis of emphysema: it may be accurate but is it relewant? Chest 1993; 103:329-330.

41. Webb WR, Muller NL, Naidich DP. High resolution CT of the lung. New York: Raven Press, 1992.

42 Muller NL, Miller RR. Diseases of the bronchioles: CT and histopathologic findings. Radiology 1995: 196:3-12.

43. Gevenois $P A$, De Vuyst $P, S y M$, et al. Pulmonary emphysema quantitative CT during expiration. Radiology $1996 ; 199: 825-829$.

44. Bergin C. Müller NL, Nichols DM, et al. The diagnosis of emphysema" a computed tomographicpathologic correlation. Am Rev Respir Dis 1986; 133:541-546.

45. Miller RR, Muller NL, Vedal S, Morrison NJ, Staples CA. Limitations of computed tomography in the assessment of emphysema. Am Rev Respir Dis 1989; 139:980-983.

46. Hruban RH, Meriane MA, Zerhouni EA, et al. High resolution computed tomography of inflationfixed lungs. Pathologic-radiologic correlation of centrilobular emphysema. Am Rev Respir Dis 1987; $136.935-940$.

47. Goddard PR, Nicholson EM, Lastlo $G$, Watt 1 . Computed tomography in pulmonary emphysema. Clin Radiol 1982; 33:379-387.

48 Thurlbeck WM, Dumnill MS, Hartung WA, Heard BE, Heppleston AG, Ryder RC. A comparison of three methods of measuring emphysema. Human Pathol 1970; 1:215-226.

49. Sakai If, Gamsu $G_{x} I m J G$, Ray CS. Pulmonary funtion abnormalities in patients with $\mathrm{CI}^{\mathrm{N}}$-determined emphysema. J Comput Assist Tomogr 1987: 11:963 968.

50. Morgan MDL. Detection and quantification of pulmonary emphysema by computed tomography: a window of opportunity. Thorax 1992; 47:1001-1004.

51. Foster WL, Pratt PC, Roggli VL, Godwin JD, Halvorsen RA, Putman CE. Centrilobular emphysema: CT-pathologic correlation. Radiology 1986: 159:27-32. 
CHAPTER 3

CT lung densitometry: a review 


\subsection{Theoretical background}

In computed tomography (CT) the X-ray attenuation walues of tissue are calculated from $X$-ray transmissions in many directions through the human body. The reconstructed $C$ T image is a two-dimensional representation of these $x$-ray attenuation coefficients in a cross-sectional body slice. The smallest unit composing a CT image is the individual picture element or pixel. The size of the image matrix is usually $256 \times 256$ or $512 \times 512$. In case of a 256 matrix, the CT image is made up of about 65000 pixels and in case of 512 matrix of 260000 pixels. The area of a pixel depends on the field of view and the matrix size. For instance, for a typical field of view of $320 \mathrm{~mm}$ the pixel area is $1.25 \times 1.25 \mathrm{~mm}^{2}$ in a 256 matrix and $0.625 \times 0.625 \mathrm{~mm}^{2}$ in a 512 matrix.

The volume of tissue represented by a pixel is known as the voxel. The size of a voxel depends on the image matrix, slice thickness and the size of the field of view. For a slice thickness of $1 \mathrm{~mm}$ and a $320 \mathrm{~mm}$ diameter scanned field of view, the corresponding voxel wolumes are $1.56 \mathrm{~mm}^{3}$ in a 256 matrix and $0.39 \mathrm{~mm}^{3}$ in a 512 matrix.

The $\mathrm{X}$-ray attemuation values of the various tissues present in a single voxel are effectively averaged. They are presented as CI numbers expressed in Hounsfield units (HU). These Founsfield units are established on a relative scale with the attenuation of water used as a reference and defined as $0 \mathrm{HU}$. The CT number of air is $-1000 \mathrm{HU}$ and that of bone of the order of $1000 \mathrm{HU}$. For a given soft tissue, CT numbers can be translated to density $\left(\mathrm{g} / \mathrm{cm}^{3}\right)$ using the formula: Physical density $=(\mathrm{CT}$ number $) / 1000$ +1 (1). It is easily seen that for instance a CT number of -800 HU corresponds to a tissue density of $0.2 \mathrm{~g} / \mathrm{cm}^{3}$.

\subsection{Factors influencing quantitative CT density measurements of the lung}

In theory, $\mathrm{CT}$ is a useful noninvasive method for evaluating the density of the lung. However, the use of absolute CT numbers for in wivo tissue characterization is compromised by a number of potential sources of errors: CT equipment, scan technique, image evaluation, the nonlinear partial volume effect and respiration.

\section{Crequipment}

CT scanners were traditionally not optimized for densitometry in the low density range that is of relevance for lung studies. Variation in the high voltage of the $X$-ray tube produced large changes in the CT numbers (2). Temporal drift in Cr numbers was dificult to control. The CT numbers of water, in a standard water phantom, was seen to vary by 15 HU over an 18 month period (2). Beam hardening and reconstruction artefacts caused density shifts (2). CT numbers were also sensitive to thoracic ge- 
ometry and to the position of the object in the scan field $(3,4)$. Densitometric results could not be extrapolated to other circumstances because of significant intrascanner and interscanner variability $(2-4)$.

The performance of newer CT equipment has been improved considerably. A CT scanner study showed that the linearity of Somatom Plus CT systems was quite adequate for the low attenuation studies that are relewant to investigations of lung disease (5). The temporal drift of the CT number for air over one day on the Somatom Plus scanner for instance, was found to be limited to within $1 \mathrm{HU}$. The long term stability was good. Thoracic geometry induced variations in Cl" numbers were limited to 3-4 $\mathrm{HU}(6)$. The reproducibility and accuracy for densities found for lung were within $2-3 \%(5)$.

\section{Scan technique}

The mean lung density as determined with CT is the histogram related densitometric parameter most commonly used in published studies. The mean lung density is relatively insensitive to the scan technique used. The influence of the reconstruction filter on the average CT value is generally negligible, except when data truncation occurs as is possible for the ultra-high resolution reconstruction filter (5). This filter produces a very broad histogram that can be truncated at its lower end, resulting in a slight overestimation of the average CT lung density. Section thickness does not affect the estimated mean lung density, except when the local density varies strongly or air calibration is incorrect $(5,6)$. The extent to which zoom factor and table height affect density measurements appears to be small (5). Density measurements are, of course, strongly affected by intravenous contrast administration. In 12 subjects receiving contrast a rise in mean lung density was noted from -813 to $-789 \mathrm{HU}(7)$.

The average density of the lung is thus a reasonable robust parameter. When histogram related parameters, other than the mean lung density are used for assessment of pulmonary disease, slice thickness and reconstruction filter will strongly affect the results. Narrower collimation and sharper reconstruction filters will broaden the CT number histogram (8). Consequently, the lowest 10 th percentile of the frequency distribution will show a decrease, while the highest 10 th percentile of the frequency distribution of CT numbers will show an increase in attenuation. A decrease from 10 to $2-\mathrm{mm}$ slice thickness was accompanied by an increase of the cross-sectional percentage in the density range $-900 \mathrm{HU}$ to $-1000 \mathrm{HU}$ from $9.6 \%$ to $16.1 \%$ (7).

\section{Image evaluation}

Manual analysis of lung tissue was time consuming and required considerable additional post-processing time. Reproducibility errors due to the operator's intervention 
in the evaluation process may exceed $10 \mathrm{HU}(9)$. At present, the lungs are automatically isolated using a suitable segmentation threshold in a contour tracking algorithm. Subsequently, outer pixels that are still affected by the thoracic wall or larger vessels have to be excluded by shrinking the contour to obtain truly parenchymal related data, a process called 'erosion'. Once the thoracic wall and the larger vessels are excluded, the mean lung density is nearly independent of additional erosions. The parameters that determine the thresholding and shrinking procedure can in principle be freely chosen but threshold and number of erosions have a substantial impact on the final densitometric outcome.

\section{Nonlinear partial volume effect}

The nonlinear partial volume effect arises in heterogeneous materials such as lung or foam, having density variations on the scale of the CT's sample area. It leads to a higher transmission of the X-ray beam than expected on the basis of an object with constant attenuation characteristics. For lung it was found to cause a systematic underestimation of the density by less than $0.1 \%(10)$. This density error is negligible and may be only of significance in calibration of $\mathrm{CT}$ scanners.

\section{Respiration}

Lung density is a reflection of the relative distributions of air, pulmonary tissue, blood and interstitial fluid within the lung. Lung density changes substantially with levels of in- or expiration. In healthy subjects, lung attenuation was found to increase during exhalation by $150-300 \mathrm{HU}(1,11-13)$. Therefore measurements should be interpreted with some knowledge of lung volume or respiratory level of inspiration. A spirometrically controlled CT technique has been developed offering the opportunity to obtain CT images at defined levels of inspiration (14).

\subsection{Density patterns of the normal lung}

Approximately two thirds of the volume of a normal lung is taken up by air, and the rest by blood, pulmonary tissue and interstitial fluid (15). These components are not homogeneously distributed throughout the lungs. Gravitation accounts for a density gradient between the dependent and the nondependent parts of the lungs. The dependent lung has higher attenuation because there is a preferential distribution of the pulmonary blood volume to the dependent lung. Alveoli and distal airways in the dependent lung are smaller than those in the non-dependent. 


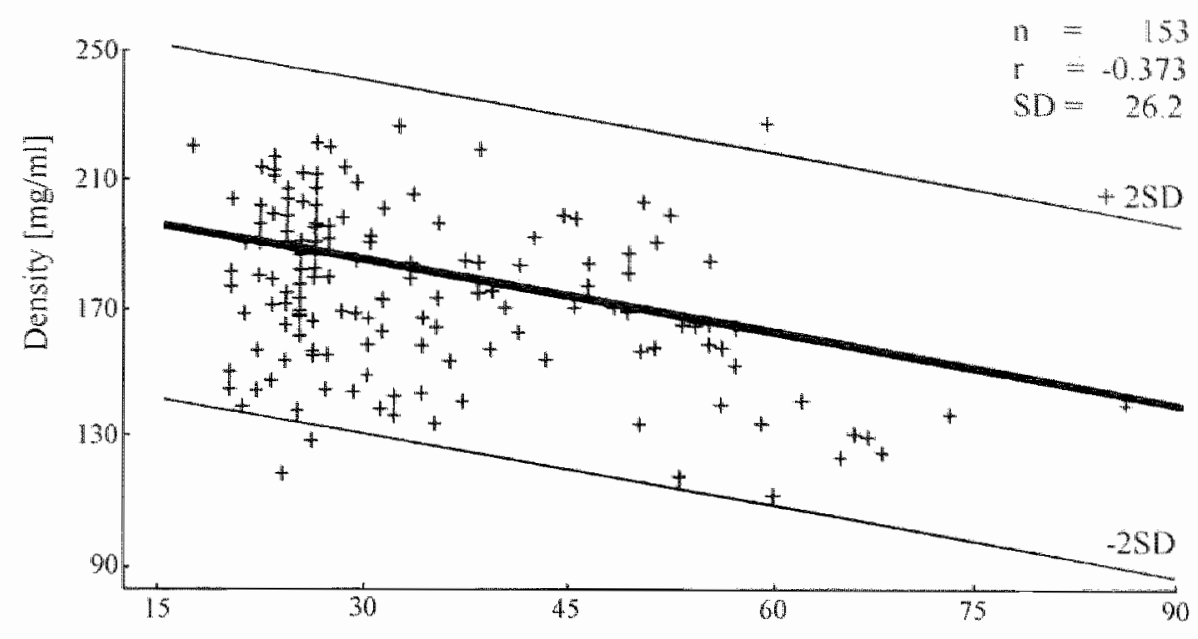

Age [Years]

Figure. Relation of lung density to age in 153 healthy individuals.

To date, suitable reference data of lung density parameters of healthy subjects (normals) are still missing. Kalender et al (16) gathered normal data from 153 healthy individuals of various ages. Three spirometrically triggered scans at a trigger level of $50 \%$ vital capacity were obtained: one at the level of the carina, one $5 \mathrm{~cm}$ above and $5 \mathrm{~cm}$ below. The authors averaged the mean lung density of these three scans. They demonstrated a decrease in lung density as a function of age at a rate of about $0.7 \mathrm{mg} / \mathrm{ml}$ per year (Figure). However, subjects in the age range between 20 and 40 years were relatively over-represented in this study.

Webb et al (13) examined 10 healthy subjects to determine normal dynamic and expiratory findings. Scans were obtained in supine and in prone positions with 3- $\mathrm{mm}$ collimation and were reconstructed with an ultra-high resonstruction algorithm. CT sections were obtained through the upper, middle and lower lungs. Attenuation was measured in the dependent and the nondependent portions of the lungs. Four of the 10 subjects showed regions of inhomogeneity in lung attenuation during rapic exhalation. In supine position, the average increase in lung attenuation during exhalation was $219 \mathrm{HU}$ in the dependent lung regions and $171 \mathrm{HU}$ in the nondependent regions. Nearly identical densitometric results were obtained in supine and prone positions.

Vock et al (12) analyzed the quantitative features of the lungs in a population of children with radiographically normal lungs. Five-mm collimated CT scans using moderate inspiration were evaluated. The mean lung density of three levels was averaged. The CT density of the lung in children ranged between $-750 \mathrm{HU}$ at the age of 10 
years $10-880 \mathrm{HU}$ at the age of 18 years. The mean lung density of 32 children over 7 years was -792 HU (95\% range, -702 to -882 HU). From maximal inspiration to expiration mean lung density increased by $158 \mathrm{HU}$. Anteroposterior gradient averaged 56 HU at the subcarinal level and increased with maximal expiration.

Density gradients beween the dependent and the nondependent lung regions are strongly influenced by lung volume. Verschakelen et al (17) studied the effect of changes in lung volume on lung density in dependent and nondependent parts of the lung. Their study group consisted of 6 healthy subjects. Spirometrically controlled CT scans were obtained at $90 \%, 50 \%$, and $10 \%$ vital capacity. Changes in lung volume caused different changes in lung density in the dependent and the nondependent parts of the lungs. The effect of lung volume was greater on the dependent than on the nondependent regions of the lungs in the upper as well as the lower zones of the lungs. The anteroposterior density gradient is less pronounced at inspiration than at expiration.

\subsection{Reproducibility of quantitative CT}

Breath hold at full inspiration is generally considered as the condition giving the best results (18). Reproducibility of mean lung density measurements under tight spirometric conditions - $50 \%$ vital capacity - in ten bealthy volunteers was on the order of $5 \%$ (14). Kohzet al (19) examined the reproducibility of densitometry in patients with pulmonary disease. One-mm collimated spirometric triggered CT scans were obtained twice, using a 5 minute break, at 50\% vital capacity, and reconstructed with a high-frequency algorithm. With tight spirometric control, the average deviation in mean attenuation values between the first and the second examination was $10 \mathrm{HU}$.

\subsection{CT lung densitometry - pathologic correlation in emphysema}

Hayhurst et al (20) calculated lung densities in CT scans of eleven patients with peripheral lung carcinoma and compared this with pathological examination of lung specimens and found that patients with emphysema had significantly more pixels in the EMII range -450 to -500 than did patients without enphysema $(p<0.001 ; 1$ EMI unit $=2 \mathrm{HU})$.

Gould et al (21) compared the lowest 5 th percentile of the lung frequency distribution of EMI numbers of two 13 - mm thick CT sections of 28 lungs of patients who proceeded to lobe or lung resection for lung tumor with the mean value of the surface area of the walls of the distal airspaces per unit lung volume (AWUV) in the pathologic specimens. They found a significant negative correlation between the lowest 5 th percentile and this AWUV $(r=-0.77, p<0.001)$. The authors concluded that quantitative 
CT scans can noninvasively diagnose, quantitate and locate mild to moderate emphysema in humans in life.

Muiller ef al (22) used a CT software program to highlight pixels within a desired density range to calculate the overall percentage of lung area of low densiry and analyzed with this program the lungs or lobes of 28 patients prior to thoracotony for lung tumor. Ten-mm CT scans were obtained at 10 - mm intervals and all patients received intravenous contrast material. The CT scans were also visually assessed for the presence and extent of emphysema. Results of both methods were compared with the results of pathological examination of the corresponding surgical specimen. The percentage CT area with attenuation less than $-910 \mathrm{HU}$ showed the highest correlation with the pathologic grade of emphysema $(r=0.94, p<0.001)$. This correlation was slightly better than the correlation between results of visual assessment and the pathological grade of emphysema ( $r=0.90, \mathrm{p}<0.001)$. The authors defined $-910 \mathrm{HU}$ as the optimal threshold value to differentiate between normal and emphysematous lung. However, because the threshold value may vary with different scanners and techniques, users were advised first to determine the optimal threshold value for their scanner.

Gevenois et al (23) analyzed CT scans of the lungs from 63 subjects undergoing surgical resection of a lung cancer or lung transplantation because of emphysema. One-mm collimated scans were obtained at 10 -nm intervals. They calculated the percentage of lung tissue with $X$-ray attenuation values lower than various threshold values and compared the results with a corresponding set of morphometric data. At inspiration, the relative area of lung parenchyma with attenuation coefficient of less than $-950 \mathrm{HU}$ correlated very well with the macroscopic severity of pulmonary emphysema $(r=0.926, p<0.001)$. The authors concluded that the relative area of lung parenchyma with attenuation coefficients of less than $-950 \mathrm{HU}$ is a valid index of macroscopic pulmonary emphysema. In a follow-up study they compared CT data at expiration and inspiration with microscopic and macroscopic morphometric methods to quantify emphysema (24) and demonstrated that $-910 \mathrm{HU}$ was a valid threshold for macroscopic and $-820 \mathrm{HU}$ for microscopic emphysema if the CT scan was made during expiration. Minus 950 HU proved to be the best threshold walue for both macroscopically and microscopically quantified emphysema if the CT scan was made during inspiration.

\subsection{CT lung densitometry-physiologic correlation in emphysema and interstitial lung disease}

Kinsella et al (25) analyzed pulmonary CT scans by calculating the percentage area of lung tissue with a density below warious density thresholds and demonstrated a significant correlation between this area and pulmonary function indices of emphysema. 
They concluded that determination of the percentage of lung areas of low $\mathrm{X}$-ray attenuation provides a useful method for quantitating the overall extent of emphysema in life.

Gould et al (26) correlated the mean density as well as lowest 5 th percentile of the lung frequency distribution of EMI numbers with measurements of airflow limitation and the diffusing capacity of the lung for carbon monoxide in 80 cigarette smokers ranging from normal subjects to patients with chronic obstructive pulmonary disease. In agreement with Kinsella inverse correlations ranging from 0.54 to 0.77 were observed. The authors concluded that decrease of CT numbers, which reflects loss of surface area of the distal airspaces, is a major index of pulmonary function in patients with smoking related chronic obstructive pulmonary disease.

Knudson et al (27) calculated the percentage of pixels in the range -900 to -1024 $\mathrm{HU}$ in 64 patients with some form of airflow obstruction. CT scans were obtained at full inspiration and expiration. The best correlation of low attenuation pixels and physiologic parameters consistent with a diagnosis of emphysema was observed on CT scans obtained at expiration. They concluded that CT scans taken at full expiration effectively revealed abnormal permanent enlargement of airspaces characteristic of emphysema. In their opinion, CT taken at full inspiration may reveal hyperaeration, which is not the same as emphysema.

Hartley et al (28) studied the value of CT densitometry in a population with interstitial lung disease. The study population consisted of 60 asbestos-exposed subjects and 24 subjects with interstitial pulmonary fibrosis. Various densitometric parameters were derived from the frequency distribution of Hounsfield numbers and correlated with accepted methods of assessing interstitial lung disease, i.e. the chest radiograph, pulmonary function testing, bronchoalveolar lavage, and dyspnea assessment. The authors concluded that computer derived density analysis is a valid objective measure of interstitial lung disease and that it may yield information beyond that available in the diagnostic tools traditionally used.

\subsection{Overview of experimental methods}

\section{CT technique used in this thesis}

CT scans were performed with a Somatom Plus scanner (Siemens, Erlangen, Germany). The method of spirometrically controlled CT densitometry of the lung we applied has been developed by Kalender and coworkers (14). The CT scanner was triggered by a respiratory gating device in order to achieve precise control of the level of inspiration. A hand-held, open spirometer system was connected to the CT scanner and to a microcomputer. In this open spirometer system, air flow causes rotation of a 
set of turbines. Body temperature pressure saturated corrections are applied. Accuracy is specified to be 2 percent (14). Flow and volume are derived by counting rotations. A spirometric curve can be followed on the display monitor of the CT scanner. Patients are placed supine on the CT couch and asked to breathe through the mouth piece. The procedure starts with a measurement of the inspiratory vital capacity. Subsequently, a user-selected level of inspiration expressed in terms of a percentage of the inspiratory vital capacity, has to be selected. When the patient inhales again to this selected level of inspiration, the microcomputer generates the trigger signals which is simultaneously sent to the spirometer and to the CT scanner. The valve in the spirometer is closed and the airflow is stopped for the duration of the CT scan and the CT scan sequence is initiated.

In each person four spirometrically gated CT scans were obtained. Two anatomical levels were chosen by means of a topogram: $5 \mathrm{~cm}$ above and $5 \mathrm{~cm}$ below the level of the carina, thereby providing representative scans of the upper and the lower parts of the lungs. At each level two scans, at 90 and 10 percent of the inspiratory vital capacity respectively, were obtained using spirometric control. The following scanning parameters were employed: 1.0 -mm collimation, $137 \mathrm{kVp}, 220 \mathrm{mAs}, 1.0$ second scanning time. We applied a 360 degree scan, a matrix size of $512 \times 512$, and a ficld of view of ap proximately $350 \mathrm{~mm}$. Scans were reconstructed in the standard or in the soft detail resolution mode. The full width at half maximum of the point spread function of the soft detail reconstruction filter is $1.86 \mathrm{~mm}$ and the sample volume $3.92 \mathrm{~mm}^{3}$ (29). No contrast medium was injected.

\section{Image evaluation}

The CT data were transmitted to a graphical workstation (SPARCstation 1+, Sun Microsystems, Mountain View, Calif) and analyzed semiautomatically with software developed in our hospital. Furst, the boundaries of each lung were determined by using a fast contour following algorithm at a CT level of -200 HU using pixel tracing, to isolate the left and the right lung parenchyma (30). Subsequently the contour was eroded by 2 pixels to remove the circumference that partially corresponded to the thoracic wall. A manual correction for excluding the trachea or separating the lungs was incidentally necessary. A frequency distribution of CI numbers was subsequently generated of the right and the left lung, and of both lungs. The area under the curve was normalized so that histograms from subjects with different lung volumes could be compared. The mean, modal, and median lung density, and the histogram"s full width at halfmaximum were determined automatically as well as the cut-off points of the frequency distribution of Hounsfield numbers defining the lowest and the highest loth percentile, and the pixel index, also called density mask, which is the percentage of the histogram area below the CT number of $-910 \mathrm{HU}$. 


\section{Dosimetry}

We determined the effective dose (E) received by the patient during the CT densitometric examination. For a scan consisting of one single section of $1 \mathrm{~mm}$ (tomogram) we determined the dose free in air using both TLD-100 chips (LiF; Solon Technologic Instruments, Wemelskirchen, Germany) and a pencil ionization chamber (Capintec PC-4P, Capintec Inc., Pittsburg) connected to a Capintec 192 A electrometer. Dose conversion factors published by GSF (31) were used to convert the central axis dose to organ doses. From these results the effective dose due to a singlle CT section scan was estimated as $\mathrm{E}=33 \mu \mathrm{Sv}$ according to IRCP-60 (32). The entrance dose due to the AP projection (topogram, for tomogram planning; technique: $120 \mathrm{kV}, 450 \mathrm{mAs}, 2-\mathrm{mm}$ collimation, $500 \mathrm{~mm}$ scan length) was measured using TLD's on a humanoid thorax phantom (33). The effective dose due to the AP-topogram was estimated at about 67 $\mu S v$, using dose conversion factors as calculated by the NRPD (34). A complete densitometric investigation consisting of 1 topogram and 4 tomograms, then caused an effective dose of $\mathrm{E}=0.20 \mathrm{mSv}$, with an estimated uncertainty of about 30 percent.

\subsection{Conclusions}

Densitometry has the potential to become an important addition to standard lung tests in determining and following the extent of emphysema and interstitial lung disease. Unfortunately the CT number histogram of the lung is very dependent on the scan parameters used. Consequently, there is an obvious need for standardization of section thickness, reconstruction filter, segmentation level, the number of erosions, not using intravenous contrast administration and the inspiratory level at which CT images are obtained.

\section{REFERENCES}

1. Rosenblum LW, Mauceri RA, Wellenstein DE, et al, Density patterns in the normal lung as determined by computed tomography. Radiology 1980; 137:409-416.

2. Zerhouni HA, Spivey JF. Morgan RH, et al. Factors influencing quantitative CT measurements of solitary pulmonary nodutes. I Comput Assist Tomogr 1982; 6:1075-1087.

3. Levi C, Gray J M. McCullough EC, Hattery RR. The unreliablity of CT numbers as absolute values. AJR $1982 ; 139: 443-447$.

4. McCullough EC, Morin RL. CT-number vartabillty in thoracic geometry. AJR 183; 141:135-140.

5. Kemerink GI, Lamers RJS, "Thelissen GRP, van Engelshoven JMA, CT densitometry of the lungs: scanner performance. I Comput Assist Tomogr 1996; 20:24-33.

6. Kemerink GJ, Lamers RIS, Thelissen GRP, van Engelshowen IMA. Scanner conformity in CT densitornetry of the lungs. Radiology 1995; 197:749-752. 
7. Adams $\mathrm{H}$, Bernard MS, McConnochie. An appraisal of CT pulmonary density mapping in nommal subjects. Clin Radiol 1991; 43:238-242.

8. Kemerink GI, Kruize HH, Lamers RJS, wan Engetshoven JMA. CT lung densitometiz: dependence of CT number histograms on sample volume and consequences for scan protocol comparability. I Comput Assist Tomogr 1997; 21:948-954.

9. Kalender WA, Fichte $\mathrm{H}$, Bautz W, Skalej M. Semiautomatic evaluation procedures for guantitative $\mathrm{CT}$ of the lung. J Comput Assist Tomogr 1991; 15:248 255.

10. Kemerink GJ, Lamers RJS, Thelissen GRP, van Engelshowen JMA. The nonlinear partial volume effect and computed tomography densitometry of foun and lung. Med Phys 1995; 22:1445-1450.

11. Zerhoun EA, Naidich DP, Stitik FP, Khouri NE, Stegelman SS. Computed tonography of the pulmonary parenchyma. II. Interstitial disease. I Thonac Imaging 1985; 1:54-64.

12. Vock P, Malanowski D, Tschaeppeler H, Kirks DR, Hedlund LW. Effmann EL. Computed tomographic lung density in children. Invest Radiol 1987; 22:627-632.

13. Webb RW, Stem ET, Kanth N, Gamsu G. Dynamic pulmonary CT: findings in healthy adult men. Ratdiology 1993; 186:117-124.

14. Kalender WA, Rienmüller R, Seissler W, Behr I, Welke M, Fichte H. Measurement of pulmonary parenchymal attenuation: use of spirometric gating with quantitative $C T$. Radiology 1990; 175:265-268.

15. Rhodes $C G$, Wollmer $P$ "Fazio F, Jones $T$. Quantitative measurement of extravascular lung density us ing positron emission and transmission tomography. I Comput Assist Tomogr 1981; 5.783-791.

16. Kalender WA, Fichte H, Bautz W, et al. Reference values for lung density and structure measured by quantitative CT. Somatom Plus Conference. Vienna 1994.

17. Verschakelen IA, Van Fraeyenhoven L, Laureys G, Demedis M, Baert AL. Differences in CT density between dependent and nondependent portions of the lung. AlR 1993; 161:713-717.

18. Wegener $\mathrm{OH}$, Koeppe P, Oeser H. Measurement of lung density by computed tomography. I Comput Assist Tomogr 1978;2:263-273.

19. Kohz P. Stabler A, Beinert T, et al. Reproducibility of quantitative, spirometrically controlled Cr. Radiology 1995; 197:539-542.

20. Hayburst MD, Flenley DC, McLean A, et al. Diagnosis of pulmonary emphysema by computerised tomography. Lancet 1984; ii:320-322.

21. Gould $\mathrm{GA}_{\text {. }}$ Macnee W, McLean A, et al. CT measurements of lung density in life can quantitate distal airspace enlargement: an essential defining feature of human emphysema. Am Rev Respir Dis 1988 ; $137.380-392$.

22. Muller NL, Staples CA, Miller RR, Abboud R"r. "Density mask": an objective method to quantitate amphysema using computed tomography. Chest 1988; 94:782-787.

23. Gevenois PA, de Maertelaer V, De Vuyst P, Zanem I. Yernault JC. Comparison of computed density and macroscopic morphometry in pulmonary emphysenta. Am J Respir Crit Care Med 1995; 152:653-657.

24. Gevenois PA, De Vuyst P, Sy M, et al. Pulmonary emphysena: quantitative CT' duringexpiration. Radiology 1996; 199:825-829.

25. Kinsella M, Müller NL, Abboud RT, Morrison NJ, DyBuncio A. Quantification of emphysema by computed tomography using a density mask program and correlation with pulmonary function tests. Chest 1990;97:315-321.

26. Gould GA, Redpath AT, Ryan M, et al. Lung CT density correlates with measurements of airlow limitation and the diffusing capacity. Eur Respir ) 1991: 4:141-146.

27. Knudson RI, Standen JR, Kaltenborn WT, el all. Expiratory computed tomography for assessment of suspected pulmonary emphysema. Chest 1991; 99:1357-1366.

28. Hartey $P G$, Galvin \R, Hunninghake $G W$, et al. High-resolution $C T$-derived measures of lung density are valid indexes of interstitial lung disease. I Appl Physiol 1994; 76:271-277.

29 Kemerink G, Kruize HH, Lamers RJS. The CT"s sample volume as a approzimate, instrumental measure for density resolution in densitometry of the lung. Med Phys 1997; 24:1615-1620. 
30. Bawlidis T. Algorithms for graphics and image evaluation. Berlme Springer Vetlag, 1982.

31. Drexler $G$, Panzer W, Widenmann $L$, Williams $G$, Zankll M. The calculation of dose from external photon exposures using reference human phantoms and Monte Carlo Methods. Part III: Organ doses in $X-$ ray diagnosis, GSF-B Bericht S-1026, 1985.

32. TCRP Publication 60. Recommendlations of the International Commission on Radiological Protection. Oxford: Pergamon Press, 1990.

33. Pearce JG, Milne ENC, Gillan GD, Roeck WW. Development of a radiographic chest phantom with disease simulation. Invest Radiol 1979; 14:181-184.

34. Hart $D$, yones $D G$, Wall BP. Estimation of effective dose in diagnostic radiology from entrance surface dose and dose-area product measurements. Chilton NRPB-R262, 1994 influence of lung wolume. AJR $1993 ; 161: 713-717$. 
CHAPTER 4

\section{Scanner conformity in CT densitometry of the lungs}

Gerrit J. Kemerink

Rob J.S. Lamers

Guillaume R.P. Thelissen

Jos M.A. van Engelshoven 


\section{ABSTRACT}

Purpose: To quantify inter- and intrascanner conformity in computed tomography (CT) densitometry of the lungs.

Materials and methods: With six scanners from four manufacturers a lung densitometry protocol with several variations was applied for performance comparison. Phantoms included water, air, and a humanoid thorax phantom equipped with a dog lung and exchangeable pseudolungs of polyethylene foam.

Results: All scanners produced acceptable CT numbers (Hounsfield units) for water, but some not for air. An incorrect calibration of air density affected all CT numbers at lung densities, but the error was easily corrected. Some systems were more sensitive to object size than others were. Sensitivity of CT numbers to section thickness, reconstruction filter, zoom factor, and table height was small, except for two scanners in relation to section thickness.

Conclusion: Alter correction for poor air calibration, scanner conformity was acceptable when the reproducibility of lung densitometry in clinical practice was set as a reference.

\section{INTRODUCTION}

Computed tomographic (CT) densitometry of the lungs is extensively used in research and is of growing clinical interest (see, eg, references 1-4 and references therein). A relevant issue for the present applications, and certainly for a more general introduction, is conformity among the various scanners on the market. In other fields of densitometry relatively poor interscanner, and even intrascanner, conformity of $\mathrm{CT}$ numbers (Hounsfield units), has been reported (5-7), Moreover, CT scanners were traditionally not optimized for the low-attenuation studies that are relevant to investigations of tung disease. To our knowledge, no systematic scanner comparison in lung densitometry has been published so far, although the scale of activities seems to warrant such an investigation. The present study reports the results from a few widespread scanners, and it addresses the problem of correcting CT-determined densities obtained with a scanner having a poor calibration at air density. 


\section{MATERIALS AND METHODS}

\section{CT-scanners}

Six CT scanners were included in the study (Table 1). All systems are third-generation scanners, except the Picker system, which is fourth-generation. With all systems scans were obtained that might be chosen for a combined densitometric and highresolution CT study. The scanning parameters chosen according to this approach are given in Table 1. Only densitometric results were evaluated. A specialist of the manufacturing or sales company of each scanner collaborated in the measurements, except in the case of the two Siemens scanners of our own hospital.

T'able 1. Scanners and Scanning Protocol.

\begin{tabular}{|c|c|c|c|c|c|c|}
\hline Manufacturer* & Scantner & $\begin{array}{l}H V^{\prime} \\
(\mathrm{kV})\end{array}$ & $\begin{array}{l}\text { Section } \\
\text { Thickness } \\
\text { (mm) }\end{array}$ & $\mathrm{mAs}$ & $\begin{array}{l}\text { Scanning } \\
\text { Duration } \\
(\mathrm{sec})\end{array}$ & $\begin{array}{l}\text { Reconstruction } \\
\text { Filter' }\end{array}$ \\
\hline $\mathrm{GE}$ & Highlight & 140 & 1.5 & 250 & 2 & STD \\
\hline Philips & SR 7000 & 140 & 1.5 & 250 & 2 & 4 \\
\hline Philips & $L X$ & 120 & 1.5 & 175 & 1.9 & 4 \\
\hline Picker & $P Q-2000$ & 130 & 1.5 & 200 & 1 & STD \\
\hline Siemens & Somatom Plus & 137 & 1.0 & 220 & 1 & AB 7055 \\
\hline Siemens & Somatom Plus & 137 & 1.0 & 220 & 1 & AB 7055 \\
\hline
\end{tabular}

Note. *GE Medical Systems, Milwaukee, Wis; Philips Medical Systems, Best, the Wetherlands; Picker International Inc, Cleveland, Ohio; Siemens Aktiengesellschaft, Erlangen, Germany; ${ }^{\text {H }} \mathrm{HV}=$ high voltage; ${ }^{*}$ All reconstruction filters are the standard type. Shown are manufacturer's code number or acronym.

\section{Phantoms}

The following phantoms were used:

1. A circular, water-filled phantom of 20-cm diameter.

2. A humanoid thorax phantom (8) containing lungs of a dog and having an empty lower thorax section that could be equipped with foam to simulate lungs. A ring of $3-\mathrm{cm}$-thick pig fat was placed around the "lean" phantom to simulate an "obese" thorax. This ring of lard was frozen to stabilize its form.

3. Various pieces of polyethylene foam ( $\mathbb{P S G}$, Wellen, Belgium) with a relatively uniform and accurately determined density (9) were used as pseudolungs in the thorax phantom. 


\section{Measurements}

The CT numbers for water and air, yielding the two calibration points of the Hounsfield scale were measured. The C $T$ number of air was measured within the empty lean and obese thorax, as well as in the empty gantry. The CT number of water was determined with the 20 - cm -diameter phantom in the middle of the gantry, by using a large circular region of interest cowering about $80 \%$ of the phantom cross section.

The dog lungs in the humanoid phantom were measured at a position that had becn marked on the outside of the phantom. The CT number was calculated as an average over a hand-drawn region completely including the left lung.

The CT numbers of the various pieces of polyethylene foam were determined with the foam in the lean and in the obese phantom. The results, consisting of CT numbers versus foam density, were fitted with the linear function $C T$ number $=$ offset + slope $x$ density. The Cr number for air (empty phantom) was included in the fit. $\chi^{2}$ analysis. was used as a measure of the quality of the fit; for a proper fit and realistic error estimates $\chi^{2}$ should be of the order of 1 .

the correlation of $C T$ number with some variations in the standard protocol was also investigated. We determined the influence of section thickness, reconstruction filter, zoom factor, and table height. In principle these parameters should not affect the average CT number, at least not for homogeneous materials. We used the water phantom, air, and the obese thorax phantom with polyethylene foam at density of 109 $\mathrm{kg} / \mathrm{m}^{3}$ (for short: foam-109). We also looked for the possible occurrence of data truncation: Some scanners do not use CT numbers below -1023 or $-1024 \mathrm{HU}$, whereas owing to noise such values may well occur for lungs of extreme low density (eg, in patients with emphysema) (9).

Assuming a well-calibrated scanner, the density of water-equivalent tissue, as lung is (10), can simply be calculated from the measured CT number by adding 1000 (strictly, the correcting at air density should be 1001.3, changing to about 998.0 at water density). The density so obtained is in kilograms per cubic meter. In the remainder of this article we use CT numbers because they are the numbers given by the scanner.

\section{RESULTS}

The CT numbers found for water, air, dog lung and foam 109 are shown in Table 2. The results for water were similar for all scanners. For air, dog lung and foam 109, the CT number with the Philips scanners deviated from the results of the other scanners. Table 3 presents the results of the measurements of foam in the lean and obese thorax phantom. The latter results are presented in the form of the offset and the slope of the linear function fitted to the data. The figure shows, as an example, the foam data 
Table 2. CT Numbers (Hounsfield units) for Water, Air, Dog Lung and Foam 109.

\begin{tabular}{|c|c|c|c|c|c|c|c|c|}
\hline Somper Gype & water & Air & $\begin{array}{l}\text { Airm Lean } \\
\text { Thorax }\end{array}$ & $\begin{array}{l}\text { Arr in Obese } \\
\text { Tirorax }\end{array}$ & Dog Lang* & $\begin{array}{l}\text { Corrected } \\
\text { Dog Lung }^{\text {B. }}\end{array}$ & 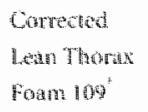 & 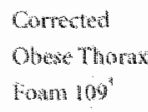 \\
\hline Highlight & +1.2 & -1008 & -1005 & -1000 & .912 & -907 & -890 & -893 \\
\hline SR 7000 & +1.0 & -984 & -985 & -985 & -887 & -902 & -896 & -896 \\
\hline$L X$ & -2.4 & -984 & -983 & -983 & -888 & -905 & -896 & -894 \\
\hline $\mathrm{PQ}-2000$ & -0.3 & -999 & -997 & -1001 & -912 & .915 & -897 & -396 \\
\hline Somatom 1 & +2.6 & -999 & -1001 & -1000 & -910 & -910 & -893 & -394 \\
\hline Somatom 2 & -1.5 & -11000 & -999 & -1000 & -911 & .911 & -894 & -894 \\
\hline
\end{tabular}

Note Scanning parameters are in Table $1{ }^{*}$ Lean thorax; ${ }^{\dagger}$ Corrected for error in CT number for air (see text).

Table 3. Measurements of Foam in the Lean and Obese Thorax.

\begin{tabular}{|c|c|c|c|c|c|c|}
\hline \multirow[b]{2}{*}{ Scanner type } & \multicolumn{3}{|c|}{ Lean Thorax } & \multicolumn{3}{|c|}{ Obese Thorax } \\
\hline & $\begin{array}{l}\text { Offset* } \\
\text { (FU) }\end{array}$ & $\begin{array}{l}\text { Slope } \\
\left(\mathrm{HU} /\left[\mathrm{kg} / \mathrm{m}^{*} \mathrm{l}\right)\right.\end{array}$ & $x^{3}$ & $\begin{array}{l}\text { Offset } \\
(\mathrm{HU})\end{array}$ & $\begin{array}{l}\text { Slope* } \\
\text { (HU/ }\left[\mathrm{kg} / \mathrm{m}^{3} \mathrm{l}\right)\end{array}$ & $x^{2}$ \\
\hline Highlight & -1005 & 0.999 & 0.64 & -1000 & 0.985 & 0.24 \\
\hline SR 7000 & -985 & 0.953 & 0.37 & -985 & 0.961 & 0.33 \\
\hline $\mathrm{LX}$ & -983 & 0.949 & 0.1 & -983 & 0.961 & 0.26 \\
\hline$P Q-2000$ & -997 & 0.950 & 0.71 & -1001 & 0.951 & 1.15 \\
\hline Somatom 1 & -1001 & 0.973 & 1.58 & -999 & 0.959 & 1.23 \\
\hline Somatom 2 & -1000 & 0.978 & 1.32 & .999 & 0.967 & 3.30 \\
\hline
\end{tabular}

Note. Scanning parameters are in Table 1; Results from a fit with the linear function CT number $=0$ flset + slope polyethyleme density.

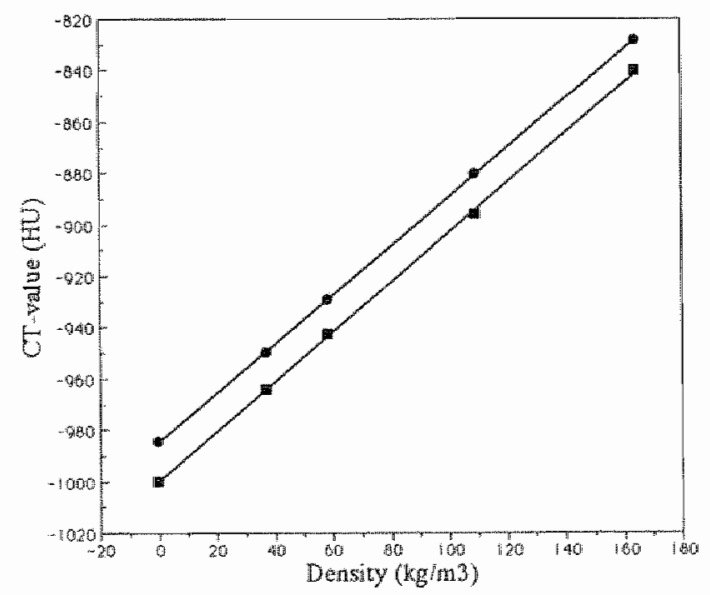

Figure. CT numbers of polyethylene form in the lean and obese thorax phantom versus polyethylene density. Experimental datta, together witls a fitted linear function, are shown for the Philips SR 7000 scanner (-) and the Siemens Somatom Plus scanner (-) (Somatom 2 in Tables 2 and 3 ). 
points, together with the line fitted to them, for the Philips SR 7000 (top) and the Siemens Somatom Plus scanner (bottom). The quality of the fit was usually good, as was also evidenced with $\chi^{2}$ value.

With all scanners, the CT numbers of air, water, and foam 109 were also measured at section thicknesses of 3,5 , and $10-\mathrm{mm}$. The Philips scanners, in contrast to the other scanners, showed considerable section thickness-dependent differences in CT numbers for air and foam. Averaging for each scanner of the non-Philips scanners the results for the four different section thicknesses, were the averaging is performed separately for air, water, and foam 109, produced standard deviations of these intrascanner averages between 0.1 and $1.2 \mathrm{HU}$. For air, the Philips SR 7000 produced -984 HU (1.5-mm section thickness), $-992 \mathrm{HU}(3 \mathrm{~mm}),-994 \mathrm{HU}(5 \mathrm{~mm})$, and $-996 \mathrm{HU}(10$ $\mathrm{mm}$ ); and the LX produced $-984 \mathrm{HU}(1.5 \mathrm{~mm}),-991 \mathrm{HU}(3 \mathrm{~mm}),-994 \mathrm{HU}(5 \mathrm{~mm})$, and $-992 \mathrm{HU}(10 \mathrm{~mm})$. Especially for sections of $1.5-\mathrm{mm}$ thickness, large deviations from the ideal value of $-1000 \mathrm{HU}$ were observed. The $\mathrm{CT}$ numbers for foam 109 showed similar shifts.

The influence of the type of reconstruction filter was normally very small: Intrascanner changes were almost always less than $1 \mathrm{HU}$, the maximum change being 2.0 HU. Greater deviations might be found in those cases in which data truncation occurs. The filters tested were, for the GE Highlight scanner, STD (standard), bone, detail, soft, and smooth; for the Picker PQ-2000 scanner: STD, sharp, and smooth; for the Philips SR 7000 and LX scanner: 4, 5,8, and 9; and for the Somatom Plus scanners: standard, high-resolution, ultra-high-resolution, and soft.

Zoom factor variation between 1.0 and 3.0 never modified CT numbers by more than $\mathbb{H U}$.

Changes in table height over a $5-\mathrm{cm}$ range never affected $\mathrm{CT}$ numbers by more than 1.5 HU. Some systems (Picker, Philips) allow scanning with the table in such a low position that it is partly outside the scan field, which leads to falsification of CT numbers (often in the form of streak artifacts). Another system (Siemens) allows only a 5 -cm vertical positioning range.

The Highlight and the Somatom Plus scanners are prone to data truncation: Data are truncated at -1024 and $-1023 \mathrm{HU}$, respectively.

\section{DISCUSSION}

The CT numbers for water indicate that all scanners tested are reasonably well adjusted at this major calibration point. For air the situation is dependent on the scanner. The Siemens Somatom Plus scanners produced values that are under all tested circumstances within 1 or $2 \mathrm{HU}$ from the ideal value of $-1000 \mathrm{HU}$. In contrast, both Philips scanners showed a deviation of slightly more than $15 \mathrm{HU}$ when a section thick- 
ness of $1.5 \mathrm{~mm}$ was used. Without correction, such a deviation induces a large error in the estimated lung density (eg, at a density of $100 \mathrm{~kg} / \mathrm{m}^{3}$ the error would be $15 \%$ ).

The GE system produces CT numbers for free air that are approximately -1008 HU, 8 HU too low. For air within the thorax phantom, the Picker and GE systems show differences on the order of 4-5 HU between data from the lean and obese thorax.

All foam data could be fitted well with a linear function, indicating that within the experimental accuracy of about $2 \%$, all tested scanners appear to be linear in the lowdensity range for a given phantom. Between the lean and obese thorax data obtained with the GE and Picker systems however, a systematic difference of 3-4 HU existed in all data points, similar to the difference observed for air. For one scanner the difference is positive; for the other, however, it is negative. In these experiments each piece of foam was measured in the lean thorax; then the ring of lard was placed in position, and with the foam lung in place, the obese thorax data were acquired. The shift in CT number must therefore be related to phantom size or phantom composition. Phantom size will affect the relative amount of scattered radiation, and phantom composition, since fat is not equivalent to water, affects beam hardening. The resulting effects in the various scanners are dependent on a number of instrumental details and calculational corrections. Factors that at least in principle are beneficial for quantitative CT are (a) relatively heavy $X$-ray beam filtration, (b) a detector diaphragm that limits section thickness, (c) a flat beam filter, and (d) good detector collimation, which is more difficult to achieve in a fourth-than in a third-generation scanner. The presence of a detector diaphragm is especially important for thin sections, but only the Somatom Plus scanners have it as a standard feature. Not only these systems, however, but also the two Philips systems showed no systematic shift in data between the lean and obese thoraxes. It is probably that these subtle effects are dependent not only on the hardware but also on the exact implementation of several corrections with the software.

The slope of the foam curves for the different scanners varies between 0.949 and $0.999 \mathrm{HU} /\left(\mathrm{kg} / \mathrm{m}^{3}\right)$. No substantial difference exists between the slopes for the lean and obese thorax data for any scanner. When the $x$-ray spectrum, filtration, and detector response of the scanner are known, the polyethylene slope can be used to obtain an estimate of the calibration curve for low-density water-equivallent tissue. In essence, under the present circumstances the ratio of the effective mass attenuation coefficients of water and polyethylene is needed for this conversion. Complications due to scanners. using a water-based beam-hardening correction are negligible, since the thickness of polyethylene is small: For all foam lungs it is less than $2.5 \mathrm{~g} / \mathrm{cm}^{2}$. For the Somatom Plus scanner the required simulations were performed (9), resulting in slopes for waterequivalent tissue that are close to the ideal value of 1.00. The calculations were not performed for the other scanners.

It is found, without exception, that the foam curves shift with the corresponding air point, as exemplified in the Figure. This finding suggests a simple method for the 
correction of $\mathrm{CT}$ numbers obtained by using a scanner with poor air calibration. It necessitates the measurement of the CT number of air, preferentially within a phantom that resembles a thorax as closely as possible, and it simply entails a correction of every measured $\mathrm{CT}$ number with the error in the air value. For example, when the air value equals $-1008 \mathrm{HU},+8 \mathrm{HU}$ is added to all measured $\mathrm{CT}$ numbers. In Table 2 the corrected $\mathrm{CT}$ numbers for the dog lung and foam 109 were calculated according to this method. Averaged over all scanners, the CT number for foam 109 in the lean thorax is $-894.3 \pm 2.4 \mathrm{HU}$, in the obese thorax, $-894.4 \pm 1.2 \mathrm{HU}$. The largest difference between the results of any two scanners was $7 \mathrm{HU}$. These results indicate a fair inter-and intrascanner conformity in densitometric estimates in the low-density range. For the dog lung, a larger spread in CT numbers was observed: The average corrected CT number was $-908.2 \pm 4.7 \mathrm{HU}$. A considerable part of the variation is due to the inhomogeneous density of the lung, and the resulting sensitivity to phantom positioning, slice thickness, and region definition. Homogeneous foam is clearly preferred for studies of this type.

A theoretically better approach to correcting poor air calibration consists in effectively making a calibration curve with an air and a water point and calculating the density according to the equation lung density $=(1000 /[$ CT number water - CT number air])(measured lung CT number - CT number air). This method should be more accurate, especially at higher densities.

Concerning the tested acquisition and reconstruction parameters that in principle should not affect CT numbers, it can be concluded that all scanners appear to be well designed. The only serious exception is the section thickness dependence of CT numbers of air for the two Philips scanners. Philips stated that they have largely solved this problem and that they started installing modifications. Substantial CT number falsification by truncation at -1023 or $-1024 \mathrm{HU}(9)$, as is possible with the Somatom Plus and the Highlight scanners, is likely to occur at extreme low densities or low mAs values, especially in combination with a high-resolution filter. When it occurs, it leads to an erroneous upward shift of the average CT number. The phenomenon is easily recognized in a histogram: At the low end, tail truncation is seen, with a peak containing the truncated pixels at the most negative CT number the system can handle. Reconstruction with a smoother filter often will remedy the problem as far as the average CT number is concerned.

The present results compare favorably with those reported in the past (5-7). Several explanations exist for this observation. First of all, the equipment has been greatly improved in the decade since the previous measurements were performed. Second, the older work concentrated on densitometry of small, nearly water-equivalent objects, sometimes within a medium of greatly different density, where partial volume effects and reconstruction filter-related effects are of importance. In the present work, 
in which CT numbers averaged over large areas are considered, these problems do not play a role.

In summary, all scanners tested have an acceptable calibration at water density. At low densities the scanners differ in their behavior. CT numbers from the GE Highlight and Picker PQ-2000 scanners show some sensitivity to phantom size or composition. The Philips scanners SR 7000 and LX have a poor, section-thickness-dependent calibration, but the systems are not very sensitive to phantom size or composition. The Siemens Somatom Plus systems are well calibrated and not very sensitive to phantom size or composition.

A simple correction for the scanners with a poor calibration at air density has been proposed. After this correction is applied, the conformity of all scanners in the lowdensity range was fair: At a density of about $100 \mathrm{~kg} / \mathrm{m}^{3}$ the standard deviation of the average over all scanners is less than $3 \mathrm{HU}$, and the maximum observed interscanner difference is $7 \mathrm{HU}$. The observed interscanner variability will generally still be smaller than the reproducibility of CT-densitometry in clinical practice $(9,11)$.

All scanners are well designed with respect to CT number sensitivity to reconstruction filter, zoom factor, table height, and section thickness, with the exception of the two Philips systems in rellation to sensitivity to section thickness.

In conclusion, when correction for poor air calibration is applied, all systems tested can be used for densitometry of the lungs, and meaningful comparison of results from the various scanners tested is possible.

\section{REFERENCES}

1. Stern EJ, Frank MS. CT of the lung in patients with pulmonary emphysema: Diagnosis, quantification, and correlation with pathologic and physiologic findings. AJR 1994; 1627911-798.

2. Rienmüler RK, Behr J, Kalender WA, et al. Standardized quantitative high resolution CT in lung diseases. J Comput Assist Tomogr 1991; 15:742-749.

3. Lamers RJS, Thellissen GRP, Kessels AG, Wouters EFM, van Engelshoven MMA. Chronic obstrictive pulmonary disease: Evaluation with spirometrically controlled CT Itng densitometry. Radiology 1994: 193:109-113.

4. Adams H, Bernard MS, McConochie K. An appraisall of CT pulmonary density mapping in normal subjects. Clin Radiol 1991; 43:238-242.

5. Levi C, GrayJE, McCullough EC, Hattery RR. The unreliability of CT numbers as absolute wal wes. AJR $1982 ; 139: 443-447$.

6. McCullough EC, Morin RL. CT-number variability in thoracic geometry. AlR 1983; 14:1:135-140.

7. Zerhouni EA, Spiwey IF, Morgan RH, Leo FP, Stitik FP, Siegelman SS. Factors infuencing quantitative CT measurements of solitary pulmonary nodules. I Comput Assist Tomogr 1982; 6:1075-1087.

8. Pearce JG, Milne ENC, Gillan GD, Roeck WW. Development of a radiographic chest phantom willy disease simulation. Invest Radiol 1979; 14:181:184.

9. Kemerink GJ, Lamers RJS, Thelissen GRP, van Engelshoven JMA. CT densitometry of the lungw" scanner performance. I Comput Assist Tomogr 1996;20:24-33. 
10. CRU report 44: Photon, electron, proton and meutron interaction data for body tissues. Bethesda, Md: International Commission on Radiation Units and Measurements, 1989.

11. Kallender WA, Rienmuller R, Seissler W, Behr I, Welke M, Fichte H. Measurement of pulmonary parenchymal atteruation: Use of spirometric gating with quantitative CT. Radiology 1990" 175:265-268. 
CHAPTER 5

\section{On segmentation of lung parenchyma in quantitative computed tomography of the lung}

Gerrit I. Kemerink

Rob ).S. Lamers

Bas J. Pellis

Han H. Kruize

Jos M.A. wan Engelshoven 


\section{ABSTRACT}

Purpose: To investigate the influence of segmentation threshold and number of erosions on parameters used in quantitative computed tomography (CT) of the lung (erosions are shrink-operations on the segmented area).

Materials and methods: Parameters assessed were mean lung density, area of the segmented lung, two percentiles and the pixel index which is the relative area of the histogram below -905 Hounsfield units (HU). We analyzed images of 10 emphysematous and 10 non-emphysematous patients, that had been scanned at carina level in inspiration and expiration, using sections of $1,2,3,5$ and $10 \mathrm{~mm}$ in combination with a standard, a smooth and an ultra-smooth reconstruction kernel. The lungs were segmented using pixel tracing at thresholds of $-200,-400$ and $-600 \mathrm{HU}$ with $0-4$ erosions, followed by histogram analysis.

Results: The area of the segmented lungs decreased with 0.9 to $3.2 \%$ per $100 \mathrm{HU}$ decrease in threshold and with 2.2 to $3.1 \%$ per erosion, dependent on patient group and level of inspiration. Estimated mean lung density changed up to $30 \%$ by changing threshold and number of erosions. The pixel index and the 10th percentile depended only slightly on threshold and number of erosions, but the 90th percentile showed a strong dependence of up to $40 \%$.

Conclusion: The segmentation protocol can have a large impact on densitometric parameters and standardization is mandatory for obtaining comparable results. Ideally a threshold equal to the average of the densities of lung and soft tissue should be used, but $-400 \mathrm{HU}$ will do in a limited but common density range $(-910$ to $-790 \mathrm{HU})$. For densitometry two erosions are recommended, for volumetry zero erosions should be used.

\section{INTRODUCTION}

Densitometry of the lung has found application without sufficient attention to methodological aspects. For instance, CT number calibration was seldomly addressed, while for several scanners considerable errors at low densities can exist (1). Although the scan protocol may affect densitometric parameters, not all relevant properties of the scan technique were usually specified. Suboptimal protocols have also been applied. It was recently shown that density resolution is an important parameter $(2-4)$ and that it is quite bad for the so called thin-section densitometry protocol, which was widely used, also by ourselves (5). Estimation of parameters that depend on the shape of the lung histogram, like the pixel index and percentiles, should not be done with this protocol $(3,4)$. Also different segmentation protocols have been applied, without assessment of the effect on densitometric results. The consequences 
of this lack of attention to methodology are suboptimal work and incomparability of most studies.

In an effort to contribute to the required methodological knowledge, this study addresses segmentation of the lung. According to the literature a few different methods have been applied for the semi-automatic segmentation of the lungs (6-10). Hedlund et al used gradients in CT numbers together with CT number ranges, both in an edge tracking method and a method that was based on the selection of contiguous pixels within the lung (6). Kalender et al introduced pixel tracing, whereby contiguous pixels in a certain CT number range were traced along the border of the lung (7). Other investigators applied methods that binarize the CT image using a threshold that discriminates lung from soft tissue $(9,10)$. When segmentation errors occur, they can be corrected manually, or, as shown by Kalender et al (7) and Zagers et al (8), by software that still may need some manual help.

A serious problem of segmentation is the inadvertent inclusion of pixels partially corresponding to soft tissue surrounding the lung. Such pixels can have a large impact on densitometric parameters of the parenchyma, especially in patients with lungs of low density as in emphysema. Although it is clear that the magnitude of the effect will depend on segmentation threshold and number of erosions, this dependence has not yet been studied. According to the literature a lew segmentation thresholds have been used: $-200 \mathrm{HU}(7),-380 \mathrm{HU}(8)$ and $-400 \mathrm{HU}(10)$. In several studies the segmentation procedure was poorly described and it was often not reported whether subsequent erosions had been applied. Even the manual of the widely used software package Pulmo CT does not specify threshold and number of erosions (7).

Unexamined so far is also the influence of segmentation threshold and spatial resolution on the area of the segmented. lung regions. The area is relevant because it is the basis for the calculation of the lung volume, which may have important applications in checking and correcting spirometry in longitudinal CT studies, and in quantifying the effect of reduction surgery. Note that spatial resolution can be varied over a wide range with section thickness and reconstruction kernel. Most studies published so far reported section thickness, but none the in-plane spatial resolution, although this latter parameter can have a significant effect on densitometric quantities (3).

In this study we investigate, for different patients and scan protocols, the effects of segmentation threshold and number of erosions on several frequently used densitometric parameters, including the area of the segmented lung. We applied a pixel tracing algorithm for automatic segmentation (11). 


\section{MATERIALS AND METHODS}

The following acquisition parameters were used on our CT scanner (Somatom Plus, Siemens, Erlangen, Germany): a non-spiral scan of $360^{\circ}$, a scan time of 1 s, a high woltage of $137 \mathrm{kV}$, a tube load of $220 \mathrm{mAs}$, the large focal spot (1.3 $\left.1.2 \mathrm{~mm}^{2}\right)$, a $512 \times 512$ matrix and a field of view of approximately $310 \mathrm{~mm}$ (zoom factor $\sim 1.6$ ) corresponding to a pixel size of about $0.6 \mathrm{~mm}$. Air callibrations were performed daily. The calibrathon of this scanner at low densities is adequate and stable over time (1).

Two groups of patients were analyzed: one group consisted of 10 persons with emphysema, the second group of 10 non-emphysematous persons with various forms of lung disease or normal lungs. The study was approved by the medical ethical committee of out institution and all patients gave informed consent. The two groups were scanned in inspiration and expiration, yielding 4 categories of data. The scans were made at the level of the carina with the patient in supine position, using section thicknesses ( 5 ) of $1,2,3,5$ and $10 \mathrm{~mm}$. Carina level was chosen because segmentation at this position can have some problems. The complete scan protocol causes an effective dose to the patient of approximately $1 \mathrm{mSv}$. All scan data were reconstructed with 3 different reconstruction kernels: standard (STD, Siemens code AB7055), soft (SFT, AB7057) and soft detail (SD, AB7059). Consequently, each of the 4 categories con sisted of 150 images. According to the CT-manufacturer the full width at half maximum (FWHM) of the point spread function (PSF) is $1.13 \mathrm{~mm}$ for the STD, $1.40 \mathrm{~mm}$ for the SFT and $1.86 \mathrm{~mm}$ for the SD kernel. For patient comfort we did not apply spirometry during the 10 scans, but we have excluded from analysis those patients whose mean density, averaged over all section thicknesses, had a large standard deviation (see below).

All images were transferred to an image processing station (ICON Power PC, Siemens Gammasonics, Hoffman Estate, IL). Using pixel tracing (7,11), the lungs were semi-automatically segmented using 3 different thresholds (T): $-200 \mathrm{HU},-400 \mathrm{HU}$ and $-600 \mathrm{HU}$. A starting point for segmentation was automatically determined by software. Values of -200 and -400 HU have been used before according to the literature; we added $-600 \mathrm{HU}$ to see the effect of a low threshold. The tracing algorithm yields a lung contour consisting of contiguous pixels with CT numbers as close as possible to the threshold, but not exceeding this. When user interaction was required the action was logged to get an impression of the frequency of the problem in relation to segmentation threshold and scan parameters. Subsequently both lung regions were subjected $100,1,2,3$ or 4 erosions (E). One erosion corresponds to the removal of all pixels lying along the circumference of the segmented area, having at least one of their eight neighbours outside the area. Histograms were created from the left lung, the right lung and the total hung, and the following histogram parameters were calculated: the area of the lung region, the mean CT number, the pixel index PI $(-905)$, the 10 th percentile $\mathrm{P}(10)$ 
and the 90 th percentile $\mathrm{P}(90)$. The mean CT number is for the (water equivalent) lung directly related to the mean density $(\rho): \rho=(1000+C T$ number $) / 1000 \mathrm{~g} / \mathrm{cm}^{3}$. Because of this simple relation we will also present density sometimes in terms of the CT number. The pixel index PI (-905) defines the relative area of the histogram below -905 $\mathrm{HU}$, and $\mathrm{P}(10)$ and $\mathrm{P}(90)$ define the $\mathrm{CT}$ number below which $10 \%$, respectively $90 \%$, of the histogram area extends. The pixel index (or density mask) has been used as a quantitative measure for emphysema (9). We chose PI $(-905)$ as the average of -900 and -910 HU which both have been used (ref's 9 and 12 and many subsequent studies).

A problem when trying to illustrate the systematic effects of segmentation threshold, number of erosions, section thickness and reconstruction kernel, is the variation in histogram parameters introduced by two other mechanisms. First, scans with different section thickness necessarily differ in the (heterogeneous) lung tissue that is sampled. Second, small differences in the degree of inspiration or expiration will exist. To reduce the impact of these two effects we included in our analysis only patients whose standard deviation of mean density, calculated from all sections in each single respiratory status, was less than $6 \mathrm{HU}$ for emphysematous patients, and less than 12 $\mathrm{HU}$ for non-emphysematous patients ( $\mathrm{T}=-400 \mathrm{HU}, \mathrm{E}=2$ ). The density is thus taken as criterium to judge constancy of inspiration and the identicalness of the tissue looked at.

Further reduction was obtained by averaging values from all patients within each of the 4 categories. This was done after largely eliminating inter-patient differences in lung properties by subtracting from each histogram parameter a patient specific reference value. This reference value was the average over all 15 combinations of section thickness and reconstruction kernel of that same parameter for $\mathrm{T}=-400 \mathrm{HU}$ and 2 erosions of that patient. Thus each patient has for each respiratory status and each histogram parameter a reference value. Averaging over 15 combinations was performed to increase the robustness of the reference value. Note that the present approach assumes that a densitometric parameter changes for all patients in a similar way around his reference value when segmentation threshold, number of erosions or spatial resolution are changed. Lung areas of each patient were expressed as a percentage of his reference area, and changes in area are given in percent-point (i.e. as simple differences between these percentages).

The advantage of averaging a histogram parameter over all patients in a given category is that trends are much more clearly reflected than they do in the data from a single patient. Therefore, this average was further used in regression analysis. Another approach to examining the large set of data would have been to perform regression analysis on the scatterplots of individual patients, and to average the slopes of all patients belonging to the same category. With this latter method essentially identical results were obtained, but less suitable graphs for visual presentation. 
According to simulations to be discussed, it is expected that for a given segmentation threshold the area is approximately linearly dependent on spatial resolution. As an effective measure for spatial resolution we used the geometric mean $R=\left(F W H M^{2} \times S\right)^{\frac{1}{3}}$, with FWHM the width of the PSF and $S$ the section thickness. This particular choice for $R$ accounts for the fact that partial volume averaging occurs in all three orthogonal directions. This $\mathrm{R}$ resulted in an approximately linear relation with lung area, and thus in a meaningful presentation of the data of all 15 combinations of kernel and section thickness in a single graph. The same way of presentation was chosen for $\rho, P I(-905)$, $P(10)$ and $P(90)$, because also rather good linearity was obtained for these parameters. For PI(-905) the quality of a linear fit, as judged from $\chi^{2}$ and visual inspection, was considerably better when $R$ was used instead of the previously used inverse square root of the sample volume (3), while for $\mathrm{P}(10)$ and $\mathrm{P}(90)$ the quality of the fit was very similar. For consistency, R was used here in all cases.

The dependence of the size of the segmented area on different reconstruction kernels and segmentation thresholds was also studied theoretically. We assumed a sharp density step between lung and the surrounding soft tissue, and convolved this step with the point spread function of the reconstruction kernels. For simplicity we assumed in most simulations that the thorax cavity locally has flat walls and that only the in-plane spatial resolution mattered. The results show the CT number dependent shift in apparent lung border with respect to the original (step) position.

We also measured the volume of a phantom that consisted of 2 polyethene bottles that had been cast into polymethylmethacrylate (PMMA or perspex). The volume of each compartment was determined by filling with water and weighing. The phantom was scanned with its long axis making angles of about 10 degrees with the axis of the scanner, both in the horizontal and in the vertical plane. The reason for this way of positioning was to get appropriate delineation of the flat bottom and top of the bottles in transversal images. Different section thicknesses, reconstruction kernels and interslice distances were applied. The zoom factor was 1.7. The volume was calculated by adding the areas of all scans and multiplying this sum with the inter-slice distance.

Due to the very large amount of lung histogram related data (we analyzed the right, the left and the total lung in 600 images) only a limited, more or less representative part will be presented. For instance, we only give results for the total lung and not for the two separate lungs. Since densitometry has been applied in particular for the quantification of emphysema during inspiration, we will generally present data for this category, but when relevant also data for the other categories will be given. 


\section{RESULTS}

\section{Manual correction of automatically generated contour}

Table 1 shows the frequency of manual operations required to correct the automatic segmentation of CT images obtained at the level of the carina. These numbers are averages over 10 patients and 15 combinations of section thickness and reconstruction kemel (thus 150 images). Figure 1. illustrates the dependence on effective spatial resom lution $(R)$ : to improve statistics we added all data of the emphysematous and nonemphysematous groups, both in inspiration and expiration, resulting in 40 cases per point. At a threshold of -600 HU the automatic segmentation was in several cases poor for non-emphysematous patients in expiration. This was caused by the relatively high density of the lung in this category (mean density $-795 \pm 73 \mathrm{HU}$, maximum $-652 \mathrm{HU}$ ), which made that parts of the lung were excluded.

\section{Area of segmented lung regions}

Figure 2 shows, for the group of emphysematous patients in inspiration, the dependence of the area of the segmented lung on segmentation threshold, effective spatial resolution and number of erosions. Note that each point is the average of 10 patients, and that each patient's value was expressed as a percentage of his reference value minus $100 \%$. For the other 3 data categories (emphysema expiration, non-emphysema inspiration and expiration) the results were qualitatively similar, except for some differences in slopes and intercepts. Table 2 gives these slopes of the scatter plots of segmented area versus effective resolution $\mathbb{R}$ for each of the 4 data categories, all 3 thresholds and zero erosions.

The dependence of the area on segmentation threshold was calculated from the difference in areas obtained for the thresholds of $-200 \mathrm{HU}$ and $-600 \mathrm{HU}$. For zero erosions one so finds: $-0.9 \pm 0.2 \%$ of the reference area per $100 \mathrm{HU}$ decrease in threshold for the emphysematous patients in inspiration, $-1.2 \pm 0.2 \%$ per $100 \mathrm{HU}$ for this group in expiration, $-1.6 \pm 0.3 \%$ per $100 \mathrm{HU}$ for the non-emphysematous patients in inspiration, and $-3.2 \pm 0.6 \%$ per $100 \mathrm{HU}$ in expiration. The change in segmented area pert erosion, for $2 \sim \mathrm{mm}$ thick sections reconstructed with the SD kernel (an average $\mathrm{R}$ ) and averaged over the 3 different thres-holds, is for the emphysematous patients in inspiration $-2.2 \pm 0.1 \%$ and in expiration $-2.5 \pm 0.1 \%$, and for non-emphysematous patients respectively $-2.4 \pm 0.1 \%$ and $-3.1 \pm 0.5 \%$. These results show that 1 erosion already has a large impact on estimated lung area, and consequently on lung volume if that would be deriwed. 
Table 1. Frequency of Manual Operations Required to Correct Automatic Segmentation

\begin{tabular}{|c|c|c|c|c|c|c|}
\hline \multirow[b]{2}{*}{ Threshold (HO) } & \multicolumn{3}{|c|}{ Inspiration } & \multicolumn{3}{|c|}{ Expiration } \\
\hline & -200 & -400 & -600 & -200 & -400 & -600 \\
\hline \multicolumn{7}{|l|}{ Emphysema } \\
\hline Separation R\&LL Lungs & 61 & 57 & 30 & 68 & 57 & 24 \\
\hline Exclusion R bronchus & 85 & 53 & 15 & 97 & 69 & 14 \\
\hline Exclusion L bronchusi & 17 & 7 & 2 & 6 & 2 & 2 \\
\hline \multicolumn{7}{|l|}{ Non-emplyysema } \\
\hline Separation R\& L lungs & 37 & 27 & 13 & 28 & 18 & 10 \\
\hline Exclusion $\mathrm{R}$ bronchus & 78 & 62 & 17 & 73 & 29 & 2 \\
\hline Exclusion L bronchus & 2 & 1 & 0 & 29 & 20 & 0 \\
\hline
\end{tabular}

Note. "Each percentuge pertains to 150 images: 10 patients, 5 section thicknesses and 3 reconstruction kernels. Data are presented as percentages.
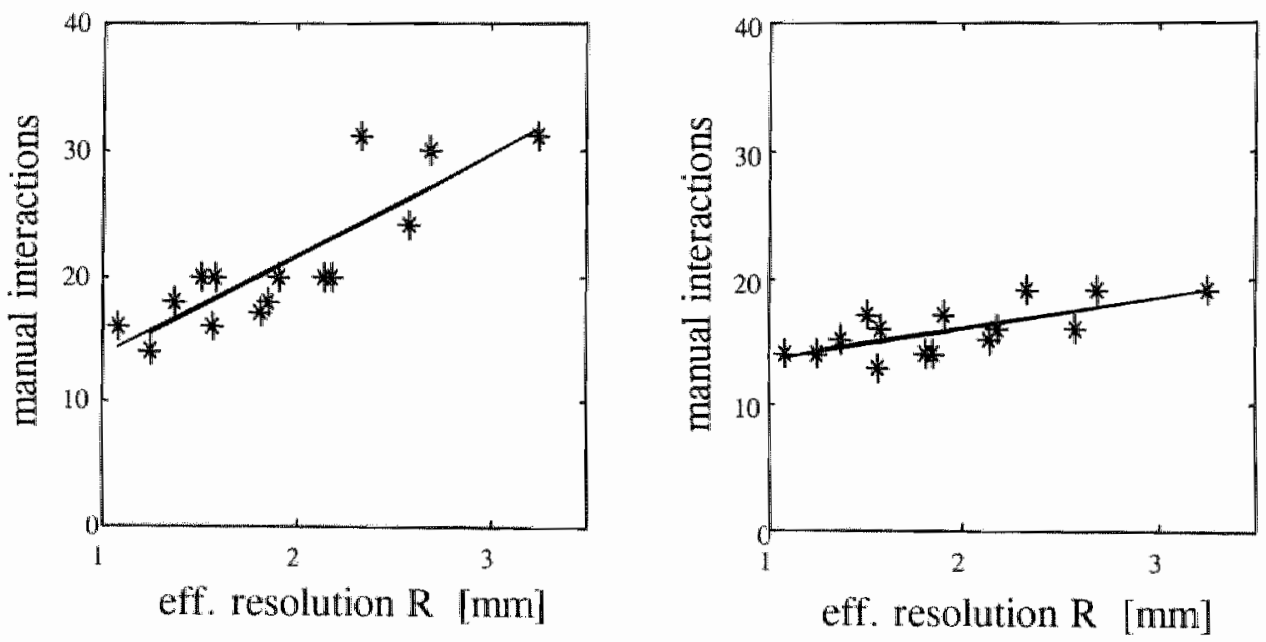

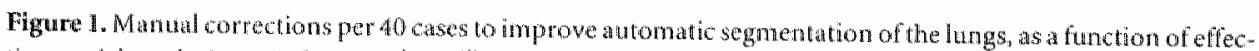

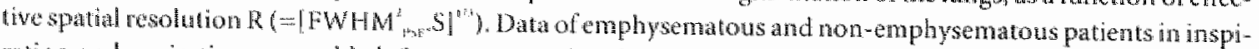
ration and expiration were added. Segmen lation theshold was -400 HU. Left. manual exclusion of trachea or right bronches, Right: manual separation of left and right lang. 


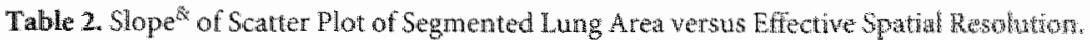

\begin{tabular}{llll}
\hline Threshold (HU) & -200 & -400 & -600 \\
\hline Emphysema & & & \\
inspiration & $0.57 \pm 0.15$ & $0.29 \pm 0.15$ & $-0.50 \pm 0.13$ \\
Expiration & $1.82 \pm 0.32$ & $1.27 \pm 0.30$ & $0.47 \pm 0.27$ \\
& & & \\
Non-emphysema & & & \\
inspiration & $0.36 \pm 0.37$ & $-0.38 \pm 0.34$ & $-1.69 \pm 0.37$ \\
Expiration & $2.80 \pm 0.54$ & $1.78 \pm 0.53$ & $-0.39 \pm 0.57$ \\
\hline
\end{tabular}

Note. ${ }^{2}$ unit of slope is percent of reference area per unit change of effective spatial resolution $(\% / \mathrm{mm}) . \mathrm{No}$ erosions thave been applied.
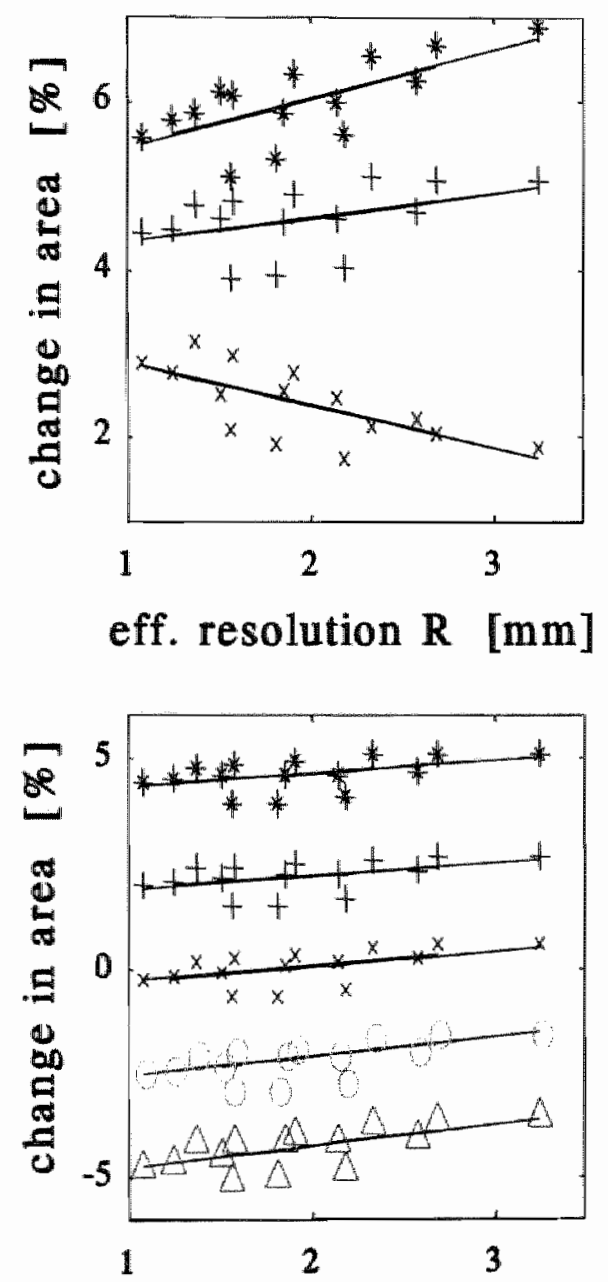

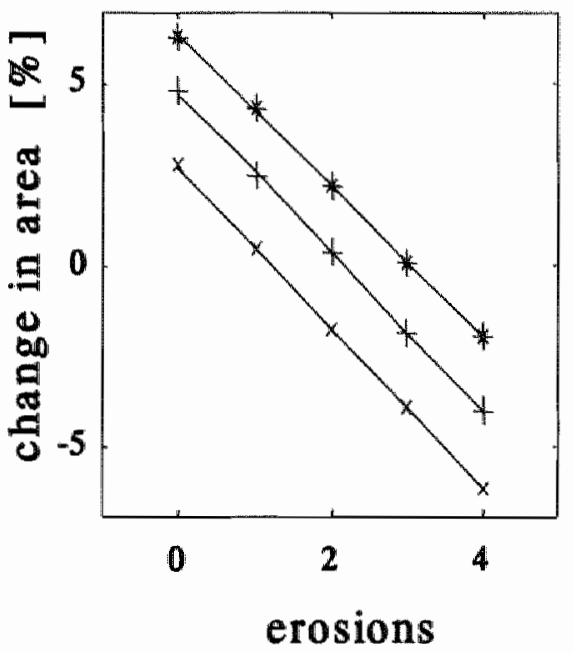

Figure 2. Percentage change in area of the to* tal segmented lung for emphysematous patients in inspiration. Data are averages over 10 patients. Lettop: Change in area as a lunetion of effectiwe spatial resolution for tor zero

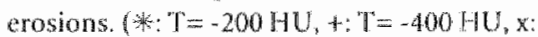
$T=-600 \mathrm{HU}$ ). Right top: Change as a function of the number of erosions for 2-mm sections and the SD kernel. (*at: $\mathrm{T}=-200 \mathrm{HU},+\mathrm{T}=$ $-400 \mathrm{HU}, \mathrm{x}: \mathrm{T}=-600 \mathrm{HU}$ ) $=$ Bottom, Change as a function of R and different numbers of erow sions for $\mathrm{T}=-400 \mathrm{HU}$. From top to bottom: from 0 to 4 rosions. Average of relerence arectas $325 \pm 54 \mathrm{~cm}^{2}$. 
Table 3 . Slope of Scatter Plot of mean CT Number of the Lung versus Effective Spatial Resolution

\begin{tabular}{llll}
\hline Erosions & $\begin{array}{l}0 \\
(\mathrm{HU} / \mathrm{mm})\end{array}$ & $\begin{array}{l}2 \\
(\mathrm{HU} / \mathrm{mm})\end{array}$ & $\begin{array}{l}4 \\
(\mathrm{HU} / \mathrm{mm})\end{array}$ \\
\hline $\begin{array}{llll}\text { Emphysema } \\
\text { inspiration }\end{array}$ & & & \\
Expiration & $6.7 \pm 0.4$ & $4.7 \pm 0.4$ & $3.1 \pm 0.4$ \\
& $4.0 \pm 0.6$ & $2.4 \pm 0.5$ & $0.6 \pm 0.4$
\end{tabular}

Non-empliysema

$\begin{array}{llll}\text { inspiration } & 6.9 \pm 0.8 & 4.9 \pm 0.8 & 2.7 \pm 0.8 \\ \text { Expiration } & 4.5 \pm 0.9 & 2.9 \pm 0.8 & 0.8 \pm 0.7\end{array}$

Note. Segmentation threshold was $-400 \mathrm{HU}$.
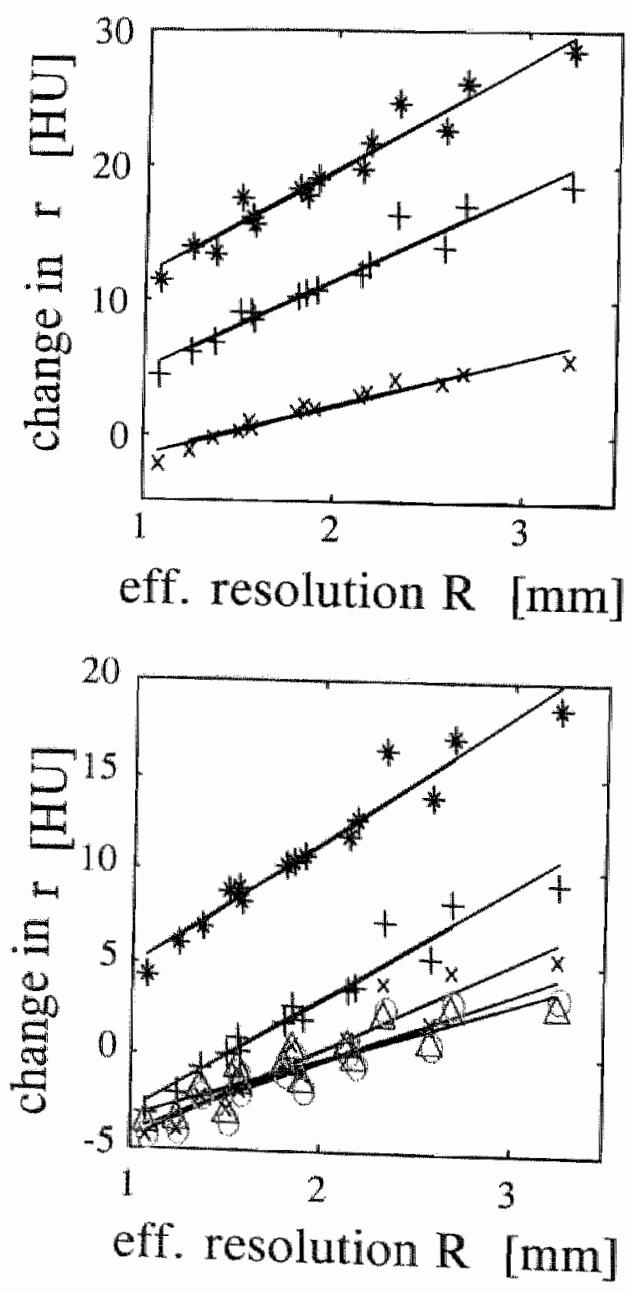

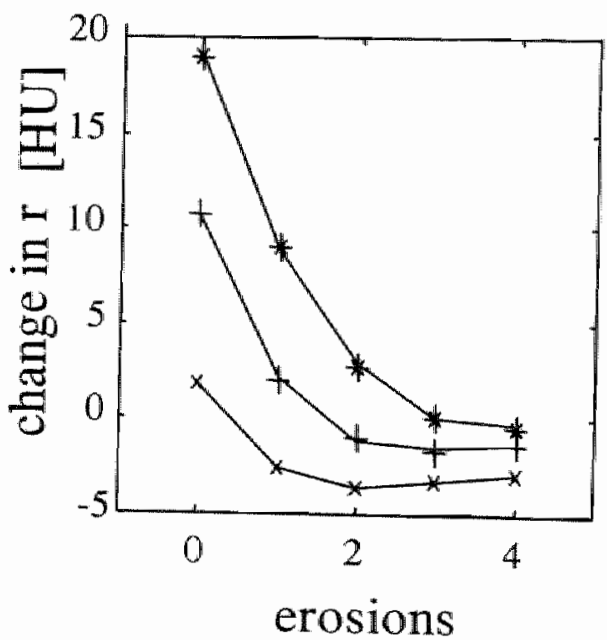

Figure 3. Clange in mean CT number of the total segmented lung for emphysematous patients in inspiration. Data are averages over 10 patients. Left top: Change in mean CT number as a function of effentive spatial resolution R for zero erosions. (* $: \mathrm{H}=-200 \mathrm{HU},+\mathrm{T}=-400 \mathrm{HU}, \mathrm{x}$ : $T=-600$ HU). Right top: Change als a function of the number of erostions for 2-mm sections and the SD kernel. (*): T= $-200 \mathrm{HU},+: T=-400 \mathrm{HU}, \mathrm{x}: \mathrm{T}=-600 \mathrm{HU})$. Bottom: Change as a function of $R$ and different numbers of erosions for $T=-400$ HU. From top to bottom: from 0 to 4 serosions. Average of reference values of mean C $\mathrm{C}$ number was $-901 \pm 14 \mathrm{HU}$. 


\section{Mean lung density}

Figure 3 shows, for the emphysematous patients in inspiration, the dependence of the mean CT number on segmentation threshold, effective spatial resolution and number of erosions. Large differences in mean density up to $30 \%$ can be obtained (the mean CT number for this category of data was $-901 \mathrm{HU}$, corresponding to $\rho=0.099 \mathrm{~g} / \mathrm{cm}^{3}$ ). Results for the other 3 data categories were similar. The slopes of the scatter plots of the mean CT number versus effective spatial resolution, for $T=-400 \mathrm{HU}$ and 0,2 and 4 erosions, are given in Table 3 . The decrease of the mean density with segmentation threshold was calculated from the differences between the mean densities obtained for the thresholds of $-200 \mathrm{HU}$ and $-600 \mathrm{HU}$. For two erosions one obtains: $-1.8 \pm 0.7 \mathrm{HU}$ per $100 \mathrm{HU}$ threshold decrease for the emphysematous patients in inspiration, $-2.6 \pm$ $0.9 \mathrm{HU}$ per $100 \mathrm{HU}$ for this group in expiration, $-3.2 \pm 1.2 \mathrm{HU}$ per $100 \mathrm{HU}$ for the nonemphysematous patients in inspiration, and $-7.0 \pm 1.2 \mathrm{HU}$ per $100 \mathrm{HU}$ in expiration.

\section{$\mathrm{PI}(-905), \mathrm{P}(10)$ and $\mathrm{P}(90)$}

Figures 4 and 5 illustrate the dependence of the pixel index PI(-905) and the percentile $\mathbb{P}(10)$ on segmentation threshold, spatial resolution and number of erosions, again for the emphysema group in inspiration. Both parameters are only slightly dependent on threshold and number of erosions. The dependence on spatial resolution is much more pronounced. The same observations hold for the other 3 data categories.

Figure 6 presents the results for the percentile $\mathrm{P}(90)$, also for the emphysema group in inspiration. Changes of more than $40 \%$ of the density corresponding to $\mathrm{P}(90)$ are possible due to changing segmentation threshold and number of erosions (average $\mathrm{P}(90)$ is $-821 \mathrm{HU}$ for this group, corresponding to a density of $0.179 \mathrm{~g} / \mathrm{cm}^{3}$ ). The slope of the scatterplot of $P(90)$ versus $R$ decreases from (on average) positive to negative when the number of erosions is sufficiently increased: for zero erosions and a threshold of $-400 \mathrm{HU}$, the slopes for each of the 4 categories are $11 \mathrm{HU} / \mathrm{mm}$ (emph., insp.), 0 $\mathrm{HU} / \mathrm{mm}$ (emph., exp.), $8 \mathrm{HU} / \mathrm{mm}$ (non-emph., insp.) and $-4 \mathrm{HU} / \mathrm{mm}$ (non-emph., exp.), while for 4 erosions the corresponding values are $-1,-9,-2$ and $-12 \mathrm{HU} / \mathrm{mm}$.

\section{Simulations and phantom study}

The results of the measurements on the two-bottle phantom are shown in Table 4. We used a segmentation threshold of $-430 \mathrm{HU}$, a value which was the average CT number for air in the bottles and the surrounding PMMA. The error in the estimated volume is small for this theoretically optimal threshold and independent of spatial resolution. 

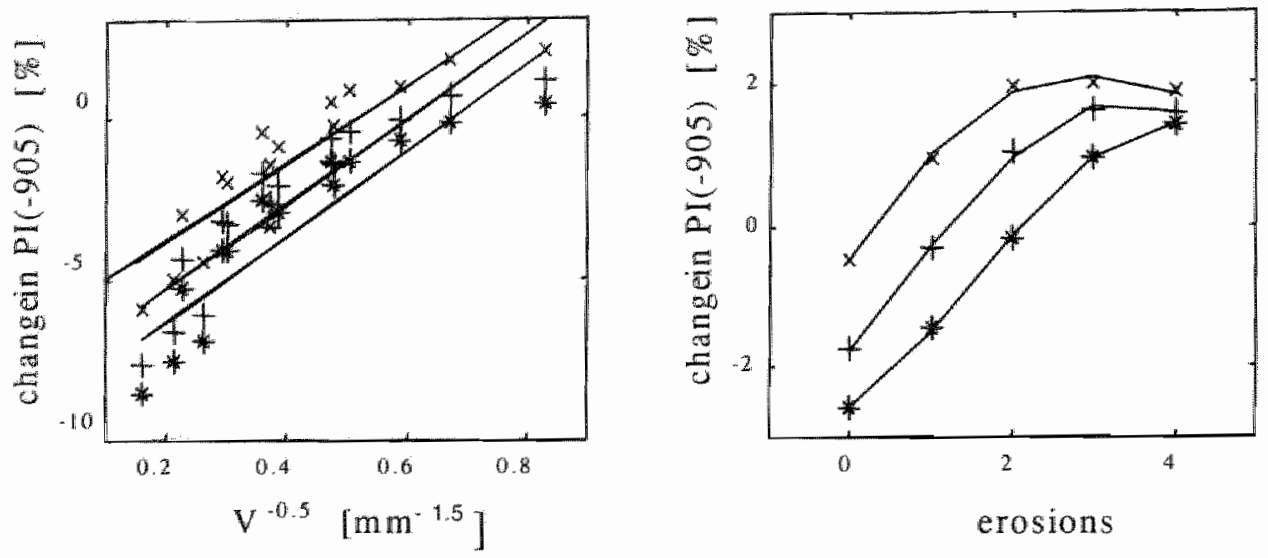

Figure 4. Change in pixel index 1 (-905) of the total segmented lung for emphysematous patients in inspiration. Data are averages over 10 patients. Left: Change in PI( -905$)$ as a function of the inwerse square root of the sample volume for zero erosions. Right: Change in PI(-905) as a function of the number of erosions for 2 -nm sections and the SD kernel. ( $* \mathrm{~T}=-200 \mathrm{HU}, t: \mathrm{T}=-400 \mathrm{HU}, \mathrm{x}: \mathrm{T}=-600 \mathrm{HU}$ ). Average of reference Pll $(-905)$ values was $64 \pm \% \%$.
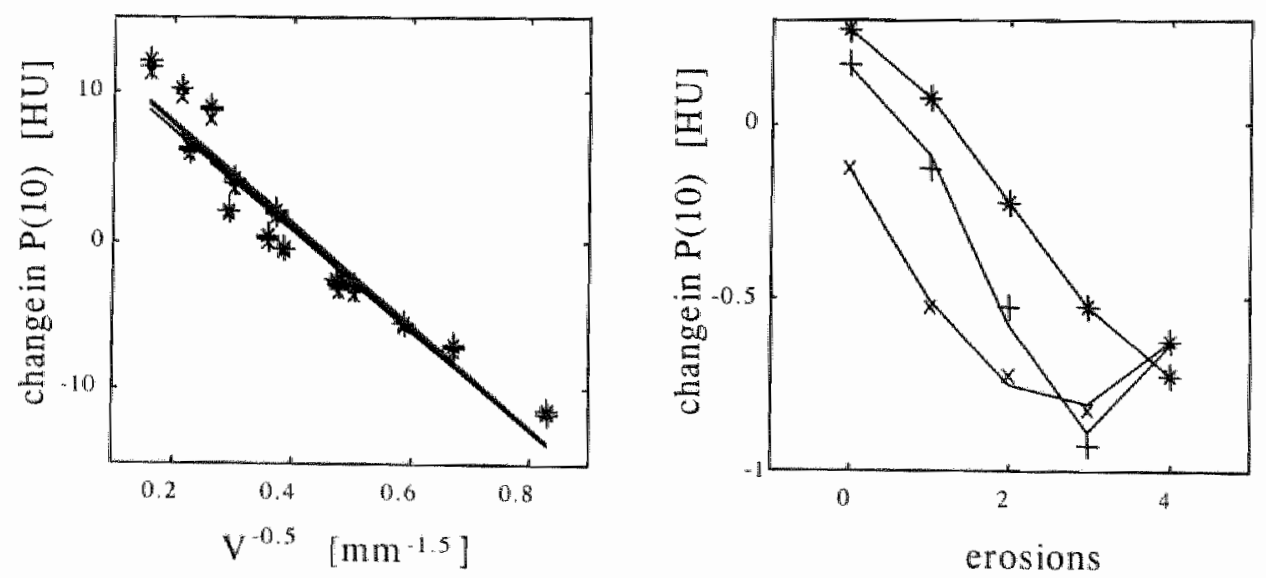

Pigure $\$$. Change in percentile $P(10)$ of the total segmented lung for enphysematous patients in inspiration. Datad are averages ower 10 patients. Let: Change in $\mathrm{P}(10)$ as a function of inverse square root of the sample volume for zero erosions. Fight: Change in $P(10)$ as a function of the number of erosions for 2 -mm sections and the SO kemel.

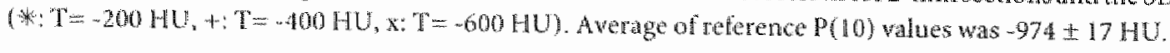



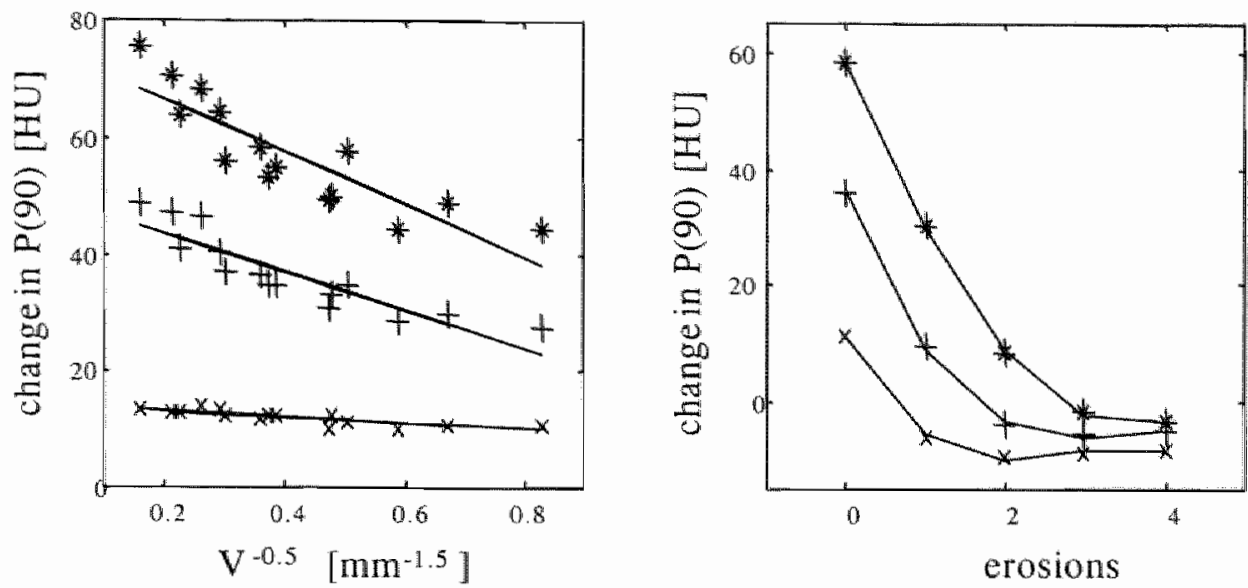

Figure 6. Change in percentile $\mathrm{P}(90)$ of the total segmented lung for emplyysematous pattients in inspiration. Data are averages over 10 patients. Left: Change in $P(90)$ as a function of the inverse square root of the sample wolune for zero erosions. Right: Change in $P(90)$ as a function of the number of erosions for 2 -mm sections and the $S D$ kernel. ( $*$ : $\mathrm{T}=-200 \mathrm{HU},+: \mathrm{T}=-400 \mathrm{HU}, \mathrm{x}: \mathrm{T}=-600 \mathrm{HU}$ ). Average of reference $\mathrm{P}(90)$ walues was $-821 \pm 15 \mathrm{HU}$.

Tablle 4. Error in Volume estimate of Two-Bottle Phantom ${ }^{2}$

\begin{tabular}{llllll}
\hline \multicolumn{5}{c}{ Section thickness } & \multicolumn{5}{l}{ error in CT estimate } \\
\cline { 3 - 6 }$(\mathrm{mm})$ & $\begin{array}{l}\text { slice distance } \\
(\mathrm{mm})\end{array}$ & recon, kernel & $\begin{array}{l}\text { left } \\
(\%)\end{array}$ & $\begin{array}{l}\text { right } \\
(\%)\end{array}$ & $\begin{array}{l}\text { total } \\
(\%)\end{array}$ \\
\hline 1 & 10 & STD & -0.72 & -0.32 & -0.52 \\
1 & 10 & SD & -0.69 & -0.26 & -0.47 \\
5 & 5 & STD & -0.33 & -0.35 & -0.34 \\
5 & 5 & SD & -0.33 & -0.40 & -0.37 \\
10 & 10 & STD & -0.01 & -0.14 & -0.06 \\
10 & 10 & SD & -0.11 & -0.05 & -0.03 \\
\hline
\end{tabular}

Note. " volumes: left bottle $2.670 \mathrm{~L}$, right $2.686 \mathrm{~L}$ and total $5.355 \pm 0.005 \mathrm{~L}$.

Figure 7 shows the results of the convolution of the point spread function of the STD and the SD reconstruction kernels with a step function representing the border between lung and soft tissue. In these simulations we assumed a CT number of lung of $-900 \mathrm{HU}$, the average for our group of emphysematous patients in inspiration, and of $50 \mathrm{HU}$ for soft tissue. The shift for a given threshold is in first approximation linearly dependent on the spatial resolution, and so is the area for shifts small compared to the linear size of the region as is here the case. Simulations with cylindrically and spherically shaped borders gave nearly identical results as long as the radii were larger than about one centimeter. 


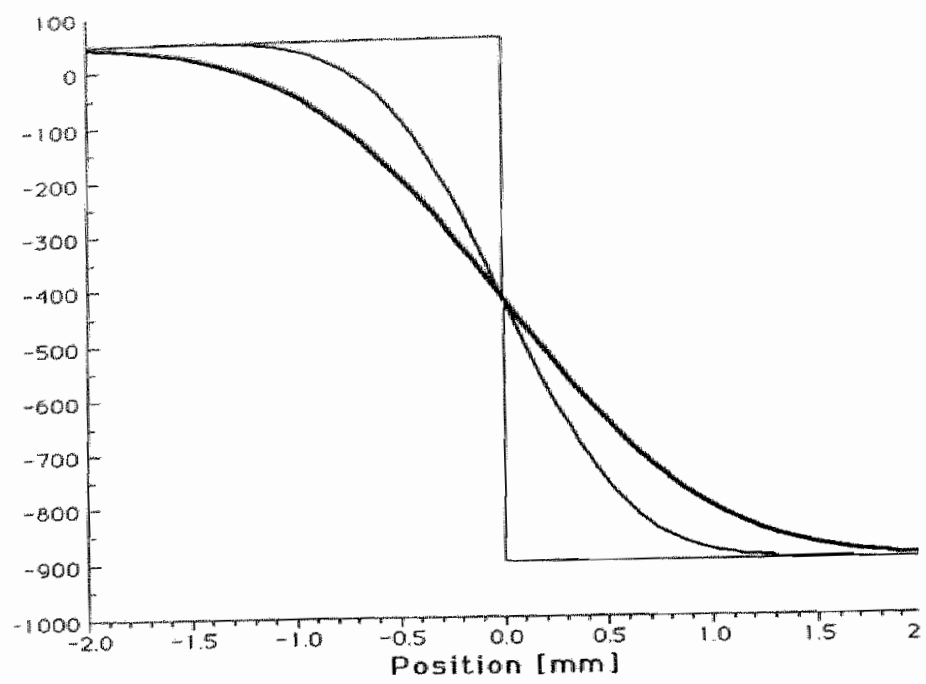

Figutre 7. Simulation of lung border in a CT image by the convolution of the point spread function of the teconstruction kernel with step border between fung (mean C. number $900 \mathrm{HU}$ ) and soft vissue (mean CT number $50 \mathrm{HU}$ ). Thin hime: border assumed in case of infinitely good spatal resolution. Medium: border obtaned

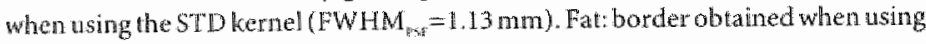
whe SD kerne! ( $P W H M$ MH $=1.86 \mathrm{~mm}$ ).

\section{DISCUSSION}

\section{Manual correction of automatically generated contours}

At carina level the number of manual corrections that is required is high, and this number increases with worsening of the spatial resolution, but decreases with decreasing threshold. These findings are in agreement with the assumption that thin septa and the finite spatial resolution, possibly in combination with some motion, are causing the failure during automatic segmentation. Note that poorer spatial resolution enhances the smearing out of small structures, thus lowering the CT values of thin septa. A low threshold is thus better suited for segmentation along thin septa, but a too low value, as our threshold of $-600 \mathrm{HU}$ for non-emphysematous patients in expiration, can lead to the exclusion of parts of the lung. At a threshold of $-400 \mathrm{HU}$ manual corrections were on average $30 \%$ less frequently required than at the more commonly used threshold of $-200 \mathrm{HU}(7)$. We did not yet implenent automatic correction algorithms, as for instance was done by Kalender et al (7) and Zagers et al (8). Note that 
Zagers et al reported that even with automatic correction between 29 and $48 \%$ of the images required user interaction.

\section{Area of segmented lung regions}

A good starting point for the discussion of the segmented lung area is Figure 7 . It shows that for a threshold that is equal to the average CT number of lung and soft tissue the position of the border of the lung is independent of spatial resolution. When a threshold is higher than this average CI number, the border will be found too far outward and the area will be overestimated. If spatial resolution is made worse in this situation, the overestimation will increase. For low thresholds the opposite occurs. Our experimental findings are largely, but not fully, in agreement with the simulations: 1. the segmented area decreases with decreasing threshold, as expected. 2. For all 4 categories the slope of the area versus spatial resolution curve decreases with decreasing segmentation threshold, and the slopes are positive for a threshold of -200 $\mathrm{HU}$, as one would expect. However, for $-600 \mathrm{HU}$ the slope is negative only for 3 out of the 4 categories, and at $-400 \mathrm{HU}$ the difference in slopes between inspiration and expiration is opposite to what one would expect. One obvious flaw of our model is that it neglects medium sized vasculature that is depicted in the CT images with a broad range of CI numbers, as a consequence of the finite resolution. These vessels can drop below the segmentation threshold when section thickness is increased or in-plane resolution is lowered. This phenomenon leads to an increase in area with poorer spatial resolution that counteracts the decrease at low thresholds that is associated with a (full) step border in other places.

From a theoretical point of view, and neglecting the complications just discussed, it is best to use a threshold that is close to the average CT number of lung and soft tissue. Our phantom study corroborates this: the volumes derived for the wo bottles were nearly identical for the STD and SD kernel, and they matched the true values quite well. At least for this phantom section thickness and reconstruction kernel are not critical for volume estimation. When the threshold deviates from the optimal value, an estimate of the systematic error can be made: a difference of $100 \mathrm{HU}$ between threshold and mean CI number of lung and soft tissue will result in an error between 0.9 and $3.2 \%$ in the area estimation, depending on patient and respiratory status. Therefore, if one would decide to use in general practice a threshold of $-400 \mathrm{HU}$, this threshold would for instance for lungs with a mean CT number between $-910 \mathrm{HU}$ and $-790 \mathrm{HU}$ never be off by more than $30 \mathrm{HU}$ from the optimal value. The systematic erw ror in the area would then generally be less than $1 \%$. In this study no mean density below-910 HU was observed, and only in the group of non-emphysematous patients 4 persons exceeded $-790 \mathrm{HU}$ in expiration (highest was $-652 \mathrm{HU}$ ). However, for volumetry it seems also possible to make a quick estimate of the mean density and to 
choose an optimal threshold accordingly. Note that in longitudinal studies random errors are probably more important than threshold related effects, at least as long the threshold is kept fixed and the same scan protocol is used. Random errors depend among others on the degree of control of inspiration and on patient positioning.

For volumetry no erosions should be applied, because I erosion already reduced the area with about $2-3 \%$. Note that these percentages are approximately propor tional to the linear dimension of a pixel.

\section{Mean lung density}

Large differences of up to $30 \%$ in estimated density could be introduced by changing segmentation threshold and number of erosions. Note that this number is for the average of 10 patients, in individual cases the effect can still be larger. These variations are caused by the inclusion or exclusion of relatively high density pixels at the circumference of the lung region. Such pixels are the result of spill over of soft tissue into the lung region by the finite resolution. They can be removed by lowering the segmentation threshold and/or applying erosions. For a given threshold, the density decreased only very slowly when more than 2 erosions were applied. Small differences for different thresholds remained, however, probably due to structures that are above the lower threshold, but below the higher. In case of the higher threshold many erosions may be required in some locations to remove these structures to the degree that they were excluded at the lower threshold. As discussed in the section on area estimation, vasculature may be involved, but also other small structures of high density (e.g. septa), as well as transitions between lung and soft tissue that appear smooth in the CT image (6).

The present results show that the estimated mean density in patient studies is dependent on spatial resolution, i.e. on section thickness and reconstruction kernel, through its effect on segmentation. The dependence is not very strong, but in previous publications we suggested (incorrectly) that there was no relation, which was based on consideration of bulk materials (2,3). This dependence is strongest when no erosions are applied, and it is reduced by eroding. For instance, for a threshold of $-400 \mathrm{HU}$ and averaging over all 4 categories of patients, the slope reduced from $5.5 \mathrm{HU} / \mathrm{mm}$ to 1.8 $\mathrm{HU} / \mathrm{mm}$ after 4 erosions. The slopes are positive because with increasing R, i.e. poorer resolution, the border profile becomes flatter so that for a given threshold more high density pixels will be included in the segmented region.

For many purposes a threshold of $-400 \mathrm{HU}$ and 2 erosions may be adequate. For lungs with very low or high density the threshold should be adapted, e.g. to the average $\mathrm{CT}$ number of lung and soft tissue as discussed above. An advantage of using $-400 \mathrm{HU}$ as a threshold, as compared to the more frequently used $-200 \mathrm{HU}$, is that the chance for successful automatic segmentation is somewhat higher. The rather widely used 
program Pulmo CT applies values that generally will be non-optimal: a threshold of $-200 \mathrm{HU}$ and 1 erosion ( 7 ).

When comparability between different investigations is mandatory, one should use the same segmentation parameters, and preferably also the same scan protocol.

$P I(-905), P(10)$ and $P(90)$

The pixel index PI(-905) and the percentile P(10) are only slightly dependent on segmentation threshold and number of erosions. Changing segmentation threshold and number of erosions affects initially only a limited number of pixels that belong to the high density part of the CT number histogram of the lung (well above $-905 \mathrm{HU}$ ). Consequently, the relative change in $\mathrm{PI}(-905)$ is roughly equal to the relative change in histogram area. For $P(10)$, when properly expressed in density, the relative change is still smaller. However, both parameters are rather strongly dependent on section thickness and reconstruction kernel (i.e. on $\mathrm{R}$ or sample volume) (3).

$P(90)$ depends heavily on segmentation threshold and number of erosions, and to a lesser degree on spatial resolution. Changes of up to $40 \%$ in the density corresponding to $P(90)$ are possible because high density pixels are involved.

The effect of decreasing resolution for uniform cellular materials is a smoother image, a narrower histogram and thus a lower $\mathrm{P}(90)$ (3). For segmented lung this effect (a negative slope of $\mathrm{P}(90)$ versus $\mathrm{R}$ ) becomes only manifest after removal of high density pixels at the circumference of the lung region by a sufficiently low threshold and enough erosions.

The dependence of PI(-905), P(10), P(90) on resolution was studied before. For the quantification of these parameters it was recommended to use a section thickness and reconstruction kernel with $\mathrm{Sx}(1.064 \mathrm{xFWHM})^{2} \geq 8 \mathrm{~mm}^{3}$, and for comparability, to adhere to a standard scan protocol $(2,3)$.

\section{Conclusions}

Most clinical densitometric studies published so far paid little attention to methodological aspects. This has led to work that was not always optimal, and to incomparability of different studies. Only with due attention to scan and analysis protocols quantitative $\mathrm{CT}$ of the lung has a chance of a more general and useful application.

In this study we investigated, for emphysematous and non-emphysematous patients and for different scan protocols, the influence of segmentation threshold and number of erosions on the following densitometric parameters: area of the segmented lung, mean lung density, the pixel index PI(-905) and the percentiles $\mathrm{P}(10)$ and $\mathrm{P}(90)$. The results indicate that different scan and segmentation protocols in principle can 
lead to unacceptably large differences in densitometric parameters. Ideally a segmentation threshold equal to the average of the densities of lung and soft tissue should be used. However, for much of the work a threshold of -400 HU will be adequate. For densitometry two erosions can be recommended, while for volumetry zero erosions should be applied. For comparability of data the same scan and analysis protocols should be applied.

\section{REFERENCES}

1. Kemerink GI, Lamers RJS, Thelissen GRP, wan Engelshowen IMA. Scanner conformity in CT densitometry of the lungs. Radiology 1995; 197:749-752.

2. Kemerink GI, Kruize HH, Lamers RIS, van Engelshoven MA. Density resolution in quantitative computed tomography of foam and lung. Med phys 1996;23:1697-1708.

3. Kemerink GI, Kruize HH, Lamers RJS, van Engelshoven JMA, CT lung densitometry: dependence of CT numbers histograms on sample volume and consequences for scan protocol comparability. I Comput Assist Tomogr 1997;21:948-954.

4. Kemerink G], Kruize HH, Lamers RIS. The CT's sample volume as an approximate, instrumental measure for density resolution in densitometry of the lung. Med Phys 1997; 24:1615-1620.

5. Lamers RIS, Thelissen GRP, Kessels AG, Wouters EFM, van Engelshoven IMA. Chronic obstructive pulmonary disease: evaluation with spironetrically controlled $\mathrm{CT}$ lung densitometry. Radiology 1994: 193:109-113.

6. Hedlund LW, Anderson RE, Goulding PL, Beck JW, Effmann EL, Putman CE. Two methods for isom lating the lung area of a CT scan for density information. Radiology 1982; 144:353-357.

7. Kalender WA, Fichte $\mathrm{H}$, Bautz W, Skalej M. Semiautomaticevaluation procedures for quantitative $\mathrm{CT}$ of the lung, J Comput Assist Tomogr 1991; 15:248-255.

8. Zagers R, Vrooman HA, Aarts NJM, et al Quantitative analysis of computed tomography scans of the lungs for the diagnosis of pulmonary emphysema. Inwest Radiol 1995; 30:552-562.

9. Müller NL, Staples CA, Miller RR, Abboud RT, "Density Mask": an objective method to quantitate emphysema using computed tomography. Chesit 1988; 94:782-787.

10. Hae KT, Slone RM, Gierada DS, Yusen RD, Cooper JD. Patients with emphysema: quantitative CI analysis before and after lung volume reduction surgery. Radiology 1997; 203:705-714.

11. Pavlidis T. Algorithms for graphics and image processing Berlin: Springer Verlag 1982.

12. Haylhurst MD. Flenley DC, MCLean $A$, et al. Diagnosis of pulmonary emphysema by computerised tomography. Lancet 198:4:2:320-322. 
CHAPTER 6

\section{Reproducibility of spirometrically controlled CT lung densitometry in a clinical setting}

Rob I.S. Lamers

Gerrit ). Kemerink

Marjolein Drent

Jos M.A. van Engelshoven 


\section{ABSTRACT}

Purpose: The aim of this study was to assess the reproducibility of spirometrically gated computed tomographic (CT) lung densitometry at defined levels of inspiration in hospitalized patients.

Materials and methods: On two consecutive days, spirometric gated CT sections were obtained from twenty hospitalized patients at $5 \mathrm{~cm}$ above and $5 \mathrm{~cm}$ below the carina, and at $90 \%$ and $10 \%$ of the vital capacity (VC). The mean, modal, and median lung densities were calculated, the cut-off points of the frequency distribution of Hounsfield units (HU) defining the lowest and the highest 10th percentile, as well as the histogram full width at half maximum. The lung density parameters of corresponding CT sections of both studies were compared. Reproducibility was expressed as the standard deviation of the signed difference between the results of day 1 and day 2 divided by $\sqrt{2}$. Reproducibility data were correlated with results of airflow limitation.

Results: At $90 \%$ VC, reproducibility in both lung zones was on the order of 3-14 HU. At $10 \% \mathrm{VC}$, reproducibility was worse by approximately a factor of three. No relationship was found between reproducibility and results of airflow limitation.

Conclusion: Objective measurement of lung density at spirometrically controlled levels of inspiration is a reproducible method in assessing pulmonary density. Reproducibility of lung density measurements is not influenced by very severe respiratory insufficiency. The most reproducible computed tomographic lung density measurements can be obtained at $90 \%$ vital capacity.

\section{INTRODUCTION}

Densitometry of the lung is extensively used in pulmonary research. It has the potential to become an important addition to standard lung function tests in diagnosing and following the extent of pulmonary emphysema and interstitial lung disease. For recent reviews of this extensive work we refer to the literature (1-2). The value of the technique is clearly proven in pulmonary emphysema. During inspiration computed tomography (CT) correlates well with the pathologic score of emphysema (3). Expiratory $\mathrm{CT}$ reflects airway obstruction and air-trapping more than it does emphysema $(4,5)$. Densitometric data may contribute to improved patient selection and to better evaluation of the response to volume reduction surgery $(6,7)$. Densitometry may provide an additional measure in selecting the side of greatest severity for unilateral lung reduction surgery (7).

The methodology of CT densitometry has been established as well as factors affecting parameters derived from a histogram of Hounsfield numbers (HU). The accuracy and scanner conformity of modern CT scanners have proved to be adequate after cor- 
rection for poor air calibration $(8,9)$. CT number histograms of the lung are strongly dependent on section thickness and reconstruction filter (10-12). To optimize CT evaluation procedures, semi-automatic algorithms isolating lung parenchyma by fast contour tracking have been developed, as well as automatic evaluation algorithms calculating various densitometric parameters automatically. Usually, mean lung density is determined as an average over a relatively large area. Unfortunately, it can be spuriously misleading especially at localized disease onset (13). Mean lung density, however, is not the only available densitometric parameter which can be extracted from the CT data. Pixel index analysis $(3,4,14)$ and the lowest 10 th percentile of the frequency distribution of densities (15) have shown promising results in the assessment of emphysema.

If densitometry of the lung is used for diagnosis and follow-up of pulmonary emphysema or interstitial lung disease, the overall precision of the procedure has to be established. Reproducibility errors due to instrumental and technical factors have generally been considered to be smaller than patient-related factors. The CT density of the lung is considerably influenced by the level of inspiration at which the CT images are obtained. Control and monitoring of this level of inspiration during scanning is of major importance to obtain reproducible CT lung density measurements. A spirometrically controlled CT technique has been developed, offering the opportunity to obtain CT scans at defined levels of inspiration (16).

The purpose of this study was to assess the reproducibility of a number of histogram-related parameters of single CT slices through the upper and the lower zones of the lungs at $90 \%$ and $10 \%$ of the vital capacity (VC) in a hospitalized patient population.

\section{MATERIALS AND METHODS}

Twenty hospitalized patients without the presence of pneumonia or lung tumor on the plain chest radiograph participated in this study. There were 11 men and 9 women, aged 18-63 yrs (median, 44 years). Ten patients had never smoked and ten were smokers or former smokers (mean consumption, 52 pack-years). Causes of hospitalization were: exacerbation chronic obstructive pulmonary disease (COPD) $(n=14)$; pulmonary embolism $(n=2)$; hemoptysis $(n=2)$; and chest wall pain $(n=2)$. The mean VC as measured at the pulmonary function laboratory was $3830 \pm 1116 \mathrm{ml}(85 \%$ pred; range $63-147 \%$ pred) and the mean forced expiratory volume in one second (FEV $)$ was $2404 \pm 1166 \mathrm{ml}$ ( $82 \%$ pred; range $30-148 \%$ pred). The study was approved by the local medical ethics committee. 


\section{Quantiative Assessment of CT scans}

Two CT studies for quantitative assessment of lung density were conducted on two separate days and were performed by two different operators. The topogram was obtained at full inspiration. Two anatomical levels were selected from the topogram: 5 cm above and $5 \mathrm{~cm}$ below the level of the carina. To control the level of inspiration during scanning, the patient was asked to breathe through a small hand held spirometer (Micro Medical Instruments, Rochester, UK), which was connected to the CT scanner (16). Patients were instructed and allowed $1 \mathrm{~min}$ to accustom to the spirometer. Subsequently the patient performed a breathing VC manoeuvre to determine the VC. At each anatomical level two scans were obtained at $90 \%$ and $10 \%$ of the inspiratory $\mathrm{VC}$, respectively. The air-flow through the spirometer was interrupted mechanically by a closing valve, at which time a trigger signal started the scanner. The level of inspiration was kept constant for the duration of the CT scan. The CT scans of the lungs were obtained in supine position with the same CT scanner (Somatom Plus; Siemens, Erlangen, Germany) with 1.0-mm collimation, $137 \mathrm{kVp}, 220 \mathrm{~mA}$, and $1 \mathrm{~s} \mathrm{scan-}$ ning time. Scans were reconstructed in the soft-detail resolution mode. The full width at half maximum of the point spread function of this reconstruction filter was $1.86 \mathrm{~mm}$ and the sample volume $3.92 \mathrm{~mm}^{3}(12)$. No intravenous contrast material was employed. All patients completed the CT study within 10 minutes.

\section{Data Analysis}

CT data were digitally transferred for analysis to a Sun SPARCstation (SUN Microsystems, Mountain View, Calif). The parenchyma of both lungs was delineated automatically by a density-discriminating computer program, which contoured at a density discriminating level of $-200 \mathrm{HU}$. Subsequently, the contour of the lung was eroded by 3 pixels. Software determined histogram shape-related parameters automatically: the mean, modal, and median lung density; the cut-off points of the frequency distribution of HU numbers defining the lowest 10 th and the highest 10 th percentile, and the histogram full width at half maximum. Densitometric parameters of the two studies were compared. Reproducibility was expressed in Hounsfield units as the standard deviation of the signed difference between results of day 1 and day 2 divided by $\sqrt{2}$ (17). This standard deviation may serve as an estimate of the variability of many repeated measurements.

\section{Statistical Analysis}

A one-sample test was used to determine whether the differences between day 1 and day 2 were statistically significant. The Shapiro-Wilk test was used to test if the differ- 
ence between the data on day 1 and day 2 could be considered as normally distributed. Relationships between reproducibility data and $\mathrm{FEV}_{1} \%$ pred were evaluated by Spearman"s rank correlation test. A probability value less than 0.05 was considered statistically significant.

\section{RESULTS}

The data derived from quantitative analysis of the CT scans are summarized in Table 1 and on reproducibility of $\mathrm{CT}$ densitometry measurements expressed in HU in Table 2. Similar differences between the various parameters derived from distributions of $\mathrm{HU}$ numbers of consecutive CT examinations were observed in the upper and the lower zones of the lungs at $90 \% \mathrm{VC}$. These differences were worse by a factor of three at $10 \%$ $\mathrm{VC}$. At $90 \% \mathrm{VC}$, the relative difference of the mean lung density expressed as a percentage between the first and the second examination was $3 \%$ in the upper as well as the lower zones of the lungs, and $6 \%$ and $5 \%$, respectively, at $10 \% \mathrm{VC}$. "The highest 10 th percentile of the frequency distribution appeared the most prone to variation at both levels of inspiration.

According to the Shapiro-Wilk test, the difference data could be considered as normally distributed. No significant difference $(\mathrm{p}<0.05)$ existed between results from any parameter on day 1 and day 2 , except for the full width at half maximum for the data from $5 \mathrm{~cm}$ below the carina at $10 \% \mathrm{VC}$.

No statistically significant relation was found between reproducibility of $\mathrm{CT}$ densitometry and $\mathrm{FEV}_{1} \%$ pred.

\section{DISCUSSION}

The reproducibility of spirometrically controlled CT densitometry of the lung was surprisingly good. The most reproducible CT lung density measurements were obtained at $90 \%$ VC. Reproducibility was worse by a factor of three at $10 \%$ vital capacity. In the upper and the lower zones of the lungs comparable differences in measurements between two successive scans were observed. The highest 10th percentile of the frequency distribution appeared to be more prone to variation. From $90 \% \mathrm{VC}$ to $10 \%$ $\mathrm{VC}$, the mean lung density increased $80 \mathrm{HU}$ in the upper zones of the lungs and $86 \mathrm{HU}$ in the lower zones. This is much less than reported by Webb et al (18) who found an increase in attenuation of $200 \mathrm{HU}$ in healthy volunteers. Our numbers are more consistent with air-trapping and are a reflection of the study population, in which patients with COPD are well represented. 
Table 1. Average Densitometric Results at $90 \%$ and $10 \%$ Vital Capacity (VC) expressed in Hounsfield Units.

$90 \% \mathrm{VC}$ $10 \% \mathrm{VC}$

\section{Upper zones}

Mean lung density

Modal

$\begin{array}{rrrr}-877 & (23) & -797 & (53) \\ -911 & (23) & -845 & (54) \\ -900 & (22) & -825 & (54) \\ -935 & (23) & -845 & (54) \\ -809 & (30) & .691 & (57) \\ 59 & (15) & 76 & (13)\end{array}$

Median

Lowest 10th percentile

Higheast 10 th percentile

full width at half-max.

\section{Lower zones}

Mean lung densiry

Modal

$\begin{array}{rrrr}-865 & (28) & -779 & (70) \\ -914 & (23) & -847 & (60) \\ -900 & (24) & -820 & (67) \\ -940 & (23) & -889 & (57) \\ -776 & (42) & -631 & (97) \\ 64 & (14) & 196 & (26)\end{array}$

Median

Lowest 10th percentile

Highest 10th percentile

64

106

Full width at half-max.

Note. Data are presented as mean, and SD in parenthesis

Table 2. Reproducibility of Quantitative Computed Tonographic Studies of the Lung at $90 \%$ and $10 \%$ Vital Capacity (VC) expressed in Hounsfield Units.

$90 \% \mathrm{VC} \quad 10 \% \mathrm{VC}$

\section{Upper zones}

Mean lung density

Modal

3

10

Median

Lowest 10 th percentile

Highest 10th percentile

Full width at half-mix.

69

\section{Lower zones}

Mean lung density

Modal

$\begin{array}{rl}3 & 11 \\ 6 & 20 \\ 5 & 15 \\ 7 & 18 \\ 14 & 38 \\ 7 & 12\end{array}$

Lung density is determined by the relative proportions of gas, blood, extracellular fluid, and pulmonary tissue. Computerized methods using standard techniques calculate the density characteristics automatically from the frequency distribution of HU. The highest 10th percentile, the full width at half maximum, the modal lung density, and the median lung density have at present no proven clinical relevance in the quan- 
tification of pulmonary emphysema or interstitial lung disease. However, lung density parameters were chosen because they reflect different aspects of the shape of the histogram of CT numbers. The highest 10th percentile of the frequency distribution was the densitometric parameter that showed the greatest variability between successive scans. This difference is probably caused by the contour tracing algorithm used for isolating the lung. This algorithm is inspiration dependent which possibly results in a different cut-off point of central vessels.

The degree of lung inflation as measured by direct spirometry has been shown to vary markedly with identical breath holding instruction on a series of consecutive CT scans (19). In cooperative patients, breath holding at full inspiration is considered the most reproducible lung volume resulting in the lowest variation in lung density between consecutive scans (19). Submaximal efforts have major effects on lung density measurements as density values vary inversely with lung volume. Monitoring of the patient's effort will minimize patient-related errors and can be accomplished by spirometric gating of the CT scanner (16). The flow-volume curve appears on the display monitor of the CT scanner. Feedback control of the spirometric curve makes it is easier to coach patients for a truly maximal effort, especially in those who suffer from severe airflow limitation, and is of indispensable help to assess patient discomfort. Submaximal effort is easily detected and the scan can be canceled. Partly for that reason reproducibility was not influenced by very severe respiratory insufficiency. Moreover, the range of mean attenuation values from inspiration to expiration is narrower in patients with COPD than in patients with normal $\mathrm{FEV}_{1} \%$ pred values (20).

The aim of the study was to address the question of respiration control on the reproducibility of standardized CT density measurements. Since maximal efforts with spirometrically controlled $\mathrm{CT}$ in supine position may be particularly fatiguing for dyspnoeic patients, we decided to scan at $90 \%$ and $10 \%$ of the VC. If reproducibility of histogram related-parameters is satisfactory at both levels of inspiration, a free choice on grounds of clinical interest can be made. Incidentally, the densitometric procedure had to be aborted because a patient was unable to reach the $90 \% \mathrm{VC}$ or the $10 \% \mathrm{VC}$ trigger level at the end of the procedure. After a timely interruption of the spirometric procedure, density measurements could always be completed successfully after 1 minute of rest. We finished the complete spirometric procedure including the $V \mathrm{C} \mathrm{ma-}$ noeuvre within about 10 minutes.

Noninvasive measurements of lung density by quantitative CT have a great potential for diagnostic purposes and follow-up after therapy. Reproducibility aspects have to be taken into account before application of this technique in practice. At $50 \% \mathrm{VC}$ the standard deviation of the estimated mean attenuation values was after a 5 min pause $10 \mathrm{HU}$ (13). This level of inspiration is suitable to differentiate between patients suffering from idiopathic lung fibrosis and patients with COPD. However, this level is of limited value in the differentiation between chronic bronchitis, pulmonary emphy- 
sema, and healthy parenchyma. CI scans obtained at full expiration have shown to correlate well with physiologic phenomena consistent with airlow limitation $(4,5)$. Small airways disease may remain completely undetected on routine suspended full inspiration scans and become conspicuous only at suspended full expiration. Paired inspiratory-expiratory CT scans are useful for distinguishing small airways disease from healthy lungs and pulmonary emphysema (20).

There are a few potential sources of variation in the level of inspiration which could influence the reproducibility of CT density measurements. Differences between the two operators in patient coaching may have an important impact on the VC manoeuwe, the result of which is subsequently used for gating the CT scanner. Patients are more familiar with the spirometer on the second day. Although VC shows variation over the day within individuals (21), examinations were arbitrarily scheduled without taking the point of the day into account, to reflect the clinical situation as closely as possible. In patients with COPD, bronchodilatator therapy was continued between CT scans. No differences in the clinical state of the patients were reported. Differences between the data on day 1 and day 2 were normally distributed and no statistically significant difference was noted between the data from day 1 and 2 , which makes a systematic source of variation in VC measurements unlikely.

In conclusion, results of spirometrically controlled CT densitometry are highly repeatable estimates of lung density. Reproducibility of lung density measurements was not infuenced by severe respiratory insufficiency. In expiration, reproducibility was worse by a factor of three. If computer tomographic scans obtained at residual volume are used for diagnosis and follow-up, respiratory control is mandatory.

\section{REFERENCES}

1. Motgan MDL. Detection and quantification of pulmonary emphysema by computed tomography: a window of opportmity. Thorax 1992; $47: 1001 \times 1004$.

2. Gevenois PA, Yarnah JC. Can conputed tomography quantify pulmonary emptysema? Eur Respir) $1995 ; 5.84: 3=848$.

3. Gevenois PA, de Matertaer V. De Vuyst P, Zanen I, Yernault IC. Comparison of computed density and macroscopic norphometry in pulmonary emphysema. An Rev Respir Dis 1995; 152:653-657.

4. Gevenois $P A$, De Vuys $P$, SY M, al. Pulmonary emphysema: quantative CT during expiration. Radiology 1996; 199:825 829.

5. Gould $G A$, Redpath AT, Ryan $M$, etal. Lung CT density conrelates with neasurements of airfow limitation and the diffusing capacity. Eur Respir I 1991; 4:141-146.

6. Bue KT, Slone RM, Gietada DS, Yusen RD, Cooper ID. Patients with emphysena: quantitative CT analysis before and after lung volume reduction surgery. Radiology 1997; 203:705*714.

7. Hober M. Brown ML, Sciurba FC, et al. Changes in lumg volume and volume of emphysema after unilateral lung reduction surgary: analysis with CT densitometry. Radiology 1996; 201:793-797.

8. Kanerink GI, lamers RBS. Thelissen GRP, wan Engelshoven DMA. Scanner conformity in CT densitometry of the lungs. Radiology 1995; 197:749-752. 
9. Kemerink G], Lamers RS, Thelissen GRP, van Engelshoven IMA. CI densitometry of the hughs: scanner performance. I Comput Assist Tomogr 1996; 20:24-33.

10. Kemerink GJ, Kruize HH, Lamers RIS. Density resolution in quantitative computed tomography of foam and lung. Med Phys 1996; 23:1697-1708.

11. Kemerink GI, Kruize HH, Lamers RJS, van Engelshoven IMA. CT hung densitometry dependence of CT number histograms on sample wolume and consequences for scan protocol comparability. $1 \mathrm{Com}-$ put Assist Tomogr 1997; 21:948-954.

12. Kemerink GI. Kruize $\mathbb{H H}$, Lamers RJS. The CT's sample volume as an approximate, instrumental measure for density resolution in densitometry of the lungs. Med Phys 1997; 24:1615-1620.

13. Kolaz. P, Stablet A, Beinert T, et al. Reproducibility of quantitative, spirometrically controlled CT. Radiology 1995; 197:539-542.

14. Muiller NL, Staples CA, Miller RR, Abboud RT. "Density Mask": an objective method to quantitate emphysema using computed tomography. Chest 1988; 94:782-787.

15. Gould $\mathrm{GA}$, MacNee W, Mclean Ax et al. CT measurements of lung density in life can quantitate distal airspace enlargement: an essential defining feature of human emphysema. Am Rev Respir Dis 1988; $137: 380-392$.

16. Kalender WA, Rienmüller R, Seissler W, Behr J, Welke M, Fichte H. Measurement of pulmonary parenchymal attenuation: use of spirometric gating with quantitative C. Radiology 1990; 175:265-268.

17. van Hastenberg RPIM, Kemerink GJ ,Hasman $A$. On the generation of short-axis and radial long-axis slices in thallium-201 myocardial perfusion single-photon emission tomography. Eur II Nucl Med $1996 ; 23: 924-931$.

18. Webb RW, Stern EJ, Kanth N, Gamsu G. Dynamic pulmonary CT: findings in healthy adult man. Raw diology $1993 ; 186: 117-124$.

19. Robinson P, Kreel L. Pulmonary tissue attenuation with computed tomography: comparison of inspiratory and expiratory scans. / Comput Assist Tomogr 1979; 37740-748.

20. Lamers RIS, Thelissen GRP, Kessels AG, Wouters EFM, van Engelshoven JMA. Chronic obstructive pulmonary disease: evaluation with spirometrically controlled CT lung densitometry. Radiology 1994; 193:109-113.

21. American Thoracic Society. Lung function testing: selection of reference values and interpretative strategies. An Rev Respir Dis 1991; 1.44:1202-1218. 

CHAPTER 7

\section{Chronic obstructive pulmonary disease: Evaluation with spirometrically controlled CT lung densitometry}

Rob ).S. Lamers

Guillaume R.P. Thelissen

Altons G.H. Kessels

Emiel E.M. Wouters

Jos M.A. van Engelshoven 


\section{ABSTRACT}

Purpose: To compare the computed tomographic (CT) lung densitometry results in patients with chronic obstructive pulmonary disease (COPD) and in control subjects (healthy persons).

Materials and methods: Spirometrically gated CT sections at $5 \mathrm{~cm}$ above and $5 \mathrm{~cm}$ below the carina at $90 \%$ and $10 \%$ vital capacity $(\mathrm{VC})$ were imaged in patients and controls. Various densitometric parameters were derived from the CT data, and results were compared between the two levels of inspiration.

Results: Densitometric results in patients with emphysema were substantially different from those in patients with chronic bronchitis and in controls at $90 \%$ and $10 \% \mathrm{VC}$. Differences in patients with chronic bronchitis and in controls were not significant at $90 \%$ VC but were significant at $10 \% \mathrm{VC}(\mathrm{p}<0.001)$. The mean changes in densitometric paraneters between $90 \%$ and $10 \% \mathrm{VC}$ were substantially greater in controls than in patients with COPD.

Conclusion: It may be possible to classify lung disease with only two CT sections obtained at the same anatomic level, one at $90 \%$ and one at $10 \% \mathrm{VC}$, irrespective of the densitometric parameter used.

\section{INTRODUCTION}

Computed tomography (CT) is a sensitive technique for evaluation of the presence and severity of emphysema. Visual assessment of emphysema by means of thinsection CT correlates well with the pathologic grade of this disease, and thin-section $\mathrm{CT}$ may be an accurate noninvasive examination for use in the diagnosis of emphysema in vivo, although the mildest forms of emphysema may be missed (1). Because visual assessment of thin-section CT scans of the lungs is subject to interobserver and intraobserver variability, methods have been developed to calculate automatically the relevant density characteristics from the CT sections for the diagnosis of emphysema $(2-4)$. Because the status of inspiration affects the lung density characteristics, control of the level of respiration during scanning (spirometrically controlled CT) is mandatory to improve the reproducibility and accuracy of density measurements (5).

The aims of this study were (a) to compare the results of spirometrically controlled CT lung densitometry in patients with emphysema or chronic bronchitis and healthy persons at different levels of inspiration, (b) to compare results of spirometrically controlled CT densitometry of the upper and the lower zones of the lungs, $(c)$ to compare the results of spirometrically controlled CT lung densitometry and of visual assessment of thin-section CT scans of the lungs with those of pulmonary function tests, and 
(d) to correlate the results of spirometrically controlled. CT lung densitometry with those of visual assessment of thin-section CT scans of the lungs.

\section{MATERIALS AND METHODS}

Three groups of individuals participated in the study. Patients with chronic obstructive pulmonary disease were prospectively selected by one pulmonologist (E.F.M.W.). Twenty consecutive patients fulfilled the pulmonary functional criteria for emphysema (6): diffusing constant for carbon monoxide ( $\mathrm{K}_{\mathrm{CO}}$ ) less than $60 \%$ of that predicted and forced expiratory volume within 1 second $\left(\mathrm{FEV}_{1}\right)$ less than $70 \%$ of that predicted (group 1). Twenty consecutive patients fulfilled the clinical criteria for chronic bronchitis as formulated by the American Thoracic Society (7): history of productive cough on most days for at least 3 months of the year for at least 2 successive years and, accordingly, a normal $\mathrm{K}_{\mathrm{CO}}(>80 \%$ of that predicted) and an obstructive lung function impairment ( $\mathrm{FEV}_{1}<70 \%$ of that predicted) that does not change markedly over several months of observation (group 2). Results in both groups were compared with those of a group of healthy persons who had no pulmonary symptoms or history of pulmonary disease and who had normal results of lung function tests $\left(\mathrm{K}_{\mathrm{CO}}>\right.$ $80 \%$ of that predicted; $\mathrm{FEV}_{1}>80 \%$ of that predicted). Detailed smoking history and informed consent were obtained from each participant. "The study was approved by the local medical ethics committee.

\section{Pulmonary Function Studies}

Pulmonary function tests were performed within 3 months before CT densitometry. Spirometric measurements were obtained by means of a wet spirometer (Gould Pulmonet III, Sensor Medics, Bilthoven, The Netherlands); FEV 1 was expressed as a percentage of the reference values (8). Lung volumes were measured with whole-body plethysmography and were expressed as a percentage of the reference values (8). The diffusing capacity was measured by the single-breath method (Masterlab; Jaeger, Würzburg, Germany). $K_{C O}$ was calculated as diffusing capacity divided by the alveolar volume.

\section{Quantitative Assessment}

CT scans of the lungs for quantitative assessment of lung density patterns were obtained with the patients supine and with use of a commercially available scanner (SOmatom Plus; Siemens, Erlangen, Germany). Scanning parameters were 1.0 $\mathrm{mm}$ collimation, $137 \mathrm{kVP}, 220 \mathrm{~m} . \mathrm{A}$, and 1.0 -second scanning time. Scans were recon- 
structed in the standard-resolution mode. No contrast medium was injected. To control the level of inspiration during scanning, the patient was asked to breathe through a small handheld spirometer (Micro Medical Instruments, Rochester, England), which was connected to the $\mathrm{CT}$ scanner. The spirometric curve could be followed on the display monitor of the CT scanner, and measurement of the vital capacity was made and displayed on the monitor before the CT scans were obtained. At userselected levels of inspiration, the air flow through the spirometer could be inhibited by a closing valve, which triggered scanning to start. The level of inspiration was constant for the duration of CT scanning. This technique was described comprehensively by Kalender et al (5). Two anatomical levels were chosen by means of a scout view: $5 \mathrm{~cm}$ above and $5 \mathrm{~cm}$ below the level of the carina. At each level, two scans, at $10 \%$ and $90 \%$ inspiratory vital capacity, respectively, were obtained. The CT data were transferred to a graphical workstation (SUN SPARCstation I+; SUN Microsystems, Mountain View, Calif) and were analyzed. First, the bounderies of each lung were determined by a density-discriminating computer program, and both lungs were automatically focussed on. A frequency histogram of Hounsfield units (HU) was subsequently generated (Figure). The area under the curve was normalized. From the data of each scan, three spirometrically controlled CT densitometric parameters were derived: mean lung density, percentage of pixels in the -910 to $-1024 \mathrm{HU}$ range (pixel index), and cut-off point in the histogram (Hounsfield unit number) that defines the lowest 10th percentile of the histogram (Figure).

\section{Vistual Assessment}

In each person, five thin-section CT scans $(1.0-\mathrm{mm}$ collimation, $137 \mathrm{kVp}, 220 \mathrm{~mA}$, 1.0 -second scanning time, high-frequency reconstruction algorithm) were obtained during breath hold at end-inspiration: two scans of the upper zones at $3 \mathrm{~cm}$ and $6 \mathrm{~cm}$ above the carina, two scans of the lower zones at $3 \mathrm{~cm}$ and $6 \mathrm{~cm}$ below the carina, and one scan at the level of the carina. Each scan was scored independently by two observers (R.J.S.L., J.M.A.V.E.) who were not aware of clinical findings for the overall severity of emphysema according to the direct observational method of Sakai et al (9). Images were made at allevel $(-800 \mathrm{HU})$ and window width $(1600 \mathrm{HU})$ appropriate for lung detail. Each section was scored for the severity of emphysema according to a four-point scale, as follows: 0, no emphysema; 1, low-attenuation areas smaller than $5 \mathrm{~mm}$ in diameter; 2 , circumscribed low-attenuation areas larger than $5 \mathrm{~mm}$ in diameter in addition to those smaller than $5 \mathrm{~mm}$ in diameter; and 3 , diffuse low-attenuation areas without intervening normal lung or large, confluent low-attenuation areas. The extent of emphysema was scored for each section also according to a four-point scale: 1 , less than $25 \%$ cross-sectional area involvement; $2,25 \%-50 \%$ cross-sectional involvement; $3,50 \%-75 \%$ cross-sectional area involvement; and 4 , more than $75 \%$ cross-sectional 


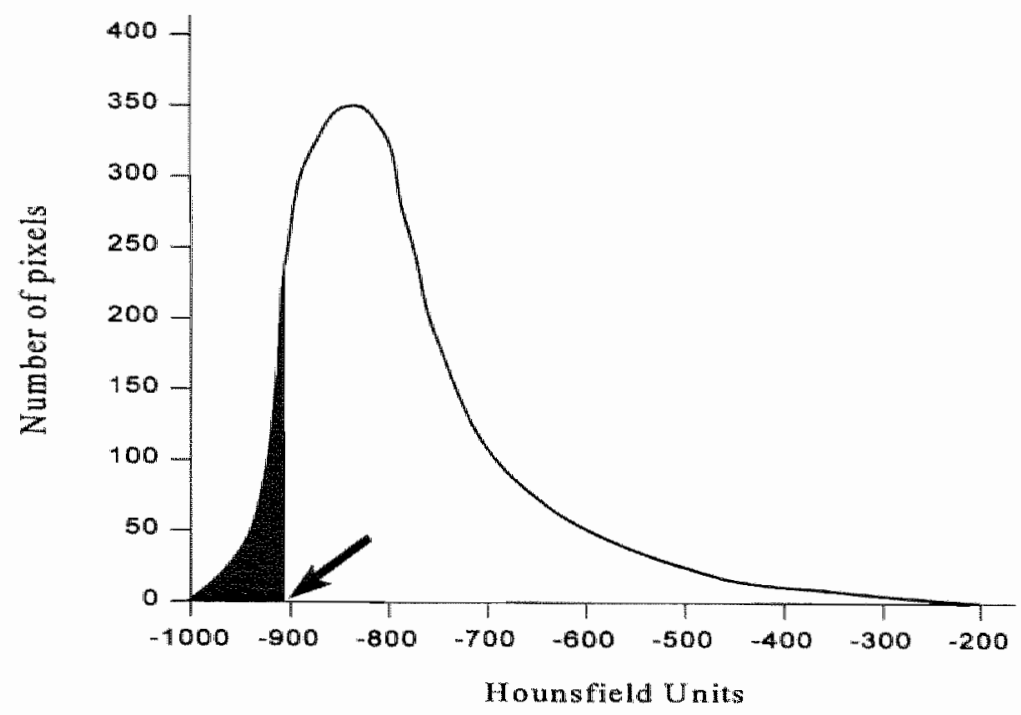

Figure. Frequency distribution of Cr numbers in the lung. Arrow marks the Hounsfield number that defines the lowest 10 th percentile; shaded area indicates the percentage of pixels that form the lowest 10 th of the frequency distribution.

area involvement. For each of the 10 lung sections, the score for severity of emplhysema was multiplied by the score for the extent, and the resultant scores were subsequently summed, which resulted in a definite emphysema score. Visual emphysema scores could range from zero to 120 . The mean visual emphysema score of both observers was calculated for the purpose of this study.

\section{Statistical Analysis}

A student $t$ test was used to determine whether differences in spirometrically controlled CT lung density parameters between the three groups at the two levels of inspiration in the upper and lower zones and between the upper and the lower zones were statistically significant. A two-tailed probability value of less than 0.05 was considered statistically significant. Correlation coefficients were calculated between the various spirometrically controlled CT lung density parameters, the mean visual emphysema score, and the results of pulmonary function tests. To determine two-tailed $p$ values for the differences of correlation coefficients in the upper zones, a bootstrap technique was used as proposed by Efron (10), because these correlation coefficients are statistically dependent. 


\section{RESULTS}

The characteristics of the three groups are summarized in Table 1 . Healthy persons were significantly younger $(\mathrm{p}<0.001)$ and their cigarette consumption was significantly lower $(\mathrm{p}<0.001)$ compared with patients with emphysema or chronic bronchitis. Weight of patients with emphysema was significantly lower $(p<0.001)$ than was weight of patients with chronic bronchitis and of healthy persons. Height did not differ in the three groups. No statistically significant difference in airway obstruction was found between the patients with emphysema and those with chronic bronchitis (FEV, $46 \%$ and $51 \%$ of that predicted, respectively). Total lung capacity in patients who had emphysema was statistically significantly higher than that of patients who had chronic bronchitis and that of healthy persons $(\mathrm{p}<0.001)$.

Table 1. Subject Characteristics.

\begin{tabular}{|c|c|c|c|c|c|c|c|c|c|c|c|c|}
\hline \multirow{3}{*}{$\begin{array}{l}\text { Parameter } \\
\text { Age }(y)\end{array}$} & \multicolumn{4}{|c|}{$\begin{array}{l}\text { Pathents with } \\
\text { Emphysemai } \\
(n=20)\end{array}$} & \multicolumn{4}{|c|}{$\begin{array}{l}\text { Patients with } \\
\text { Chronic Bronchitis } \\
(\mathrm{n}=20)\end{array}$} & \multicolumn{4}{|c|}{$\begin{array}{l}\text { Healthy Persons } \\
(n=20)\end{array}$} \\
\hline & \multicolumn{3}{|c|}{ Mean $\pm S D$} & \multirow[t]{2}{*}{$p^{*}$} & \multicolumn{3}{|c|}{ Mean $\pm S D$} & \multirow{2}{*}{ pi } & \multicolumn{3}{|c|}{ Mean $\pm S D$} & \multirow[t]{2}{*}{$p \ddagger$} \\
\hline & 60 & \pm & 8 & & 70 & \pm & 7 & & 53 & \pm & 12 & \\
\hline Height $(\mathrm{cm})$ & 171 & \pm & 8 & & 171 & \pm & 8 & & 175 & \pm & 10 & \\
\hline Weight $(\mathrm{kg})$ & 61 & \pm & 11 & $<.001$ & 76 & \pm & 11 & & 78 & \pm & 10 & \\
\hline Cig. cons. (pack-years) & 49 & \pm & 26 & & 40 & \pm & 31 & & 17 & \pm & 12 & $<.001$ \\
\hline Male gender & & 14 & & & & 19 & & & & 20 & & \\
\hline $\mathrm{FEV}_{1}(\%$ predicted $)$ & 46 & \pm & 12 & & 51 & \pm & 14 & & 110 & \pm & 18 & $<.001$ \\
\hline TVC (\% predicted) & 100 & 半 & 26 & & 87 & \pm & 18 & $<.001$ & 111 & \pm & 1.4 & \\
\hline TLLC (\% predicted) & $\| 11$ & \pm & 8 & $<001$ & 99 & \pm & 9 & & 100 & \pm & 11 & \\
\hline$D L_{, i,}(\%$ predicted) & $6 \llbracket$ & \pm & 14 & $<.00 \|$ & 100 & \pm & 19 & & 111 & \pm & 19 & \\
\hline$K_{c, i}(\%)$ predicted $)$ & 47 & \pm & 8 & $<.001$ & 95 & \pm & 12 & & 90 & \pm & 9 & \\
\hline
\end{tabular}

Note. SD = standard deviation; "Patients with emphysema versus those with chronic bronchitis; $\uparrow$ Patients with chronic bronchitis versus healthy persons; $\$$ Patients with emphysema versus healthy persons.

The mean visual emphysema score in patients with emphysema was significantly higher $(50 \pm 26)$ than that of patients with chronic bronchitis $(5 \pm 13, p<0.001)$ and that of healthy persons $(1 \pm 2, p<0.001)$, but there was no significant difference in the mean visual emphysema scores of patients with chronic bronchitis and healthy persons. All patients with emphysema had emphysematous changes that were visually assessed at thin-section CT (range, 16-116), whereas only two patients with chronic bronchitis had a mean visual emphysema score greater than 15 ( 19 and 57 , respectively). 
Table 2. Densitometric Results at 10\% and $90 \%$ Vital Capacity with the Patient supine.

\begin{tabular}{|c|c|c|c|c|c|c|}
\hline \multirow[b]{2}{*}{ Vilal Capacity $(\%)$} & \multicolumn{2}{|l|}{$\begin{array}{l}\text { Patients with } \\
\text { Emphysenga } \\
(\mathrm{n}=20)\end{array}$} & \multicolumn{2}{|c|}{$\begin{array}{l}\text { Patients with } \\
\text { Chronic Bronchitis } \\
(n=20)\end{array}$} & \multicolumn{2}{|c|}{$\begin{array}{l}\text { Healthy Persons } \\
(\mathrm{n}=20)\end{array}$} \\
\hline & Mean \pm SD & $p^{*}$ & Mean $\pm S D$ & pt & Mean $\pm S D$ & $p *$ \\
\hline \multicolumn{7}{|l|}{ Upper zones } \\
\hline \multicolumn{7}{|l|}{ Mean lung density } \\
\hline-10 & $862 \pm 27$ & $<.001$ & $-827 \pm 38$ & $<.001$ & $-786 \pm 39$ & $<.001$ \\
\hline-90 & $-886 \pm 23$ & $<.001$ & $-852 \pm 32$ & & $-858 \pm 29$ & $<.001$ \\
\hline \multicolumn{7}{|l|}{ Pixel index } \\
\hline-10 & $42 \pm 14$ & $<.001$ & $27 \pm 16$ & $<.001$ & $9 \pm 11$ & $<.001$ \\
\hline-90 & $56 \pm 12$ & $<.001$ & $38 \pm 17$ & & $41 \pm 20$ & $<.001$ \\
\hline \multicolumn{7}{|c|}{ Lowest loth perc.(HU) } \\
\hline-10 & $-967 \pm 20$ & $<.001$ & $-941 \pm 26$ & & $-893 \pm 38$ & $<.001$ \\
\hline-90 & $-977 \pm 17$ & $<.001$ & $-951 \pm 25$ & & $-948 \pm 23$ & $<.001$ \\
\hline \multicolumn{7}{|l|}{ Lower zones } \\
\hline \multicolumn{7}{|l|}{ Mean lung density } \\
\hline-10 & $-849 \pm 31$ & $<.001$ & $-809 \pm 55$ & $<.001$ & $-767 \pm 56$ & $<001$ \\
\hline-90 & $-873 \pm 24$ & $<001$ & $-843 \pm 22$ & & $-847 \pm 34$ & $<.001$ \\
\hline \multicolumn{7}{|l|}{ Pixel index } \\
\hline-10 & $41 \pm 16$ & $<.001$ & $28 \pm 14$ & $<001$ & $13 \pm 13$ & $<.001$ \\
\hline-90 & $54 \pm 12$ & $<.001$ & $39 \pm 12$ & & $42 \pm 20$ & $<001$ \\
\hline \multicolumn{7}{|c|}{ Lowest loth perc.(HU) } \\
\hline-10 & $-961 \pm 16$ & $<.001$ & $-941 \pm 21$ & $<.001$ & $-906 \pm 37$ & $<.001$ \\
\hline-90 & $-972 \pm 14$ & $<.001$ & $-953 \pm 14$ & & $-950 \pm 23$ & $<.001$ \\
\hline
\end{tabular}

Note. SD = standard dewiation; * Patients with emphysema versus those with chronic bronchitis; it Patients with chronic bronchitis versus healthy persons; $\$$ Patients with emphysema versus healthy persons.

The densitometric results in the three study groups are summarized in Table 2. At both levels of inspiration the mean lung density, pixel index, and lowest 10 th percentile of the frequency distribution in patients with emphysema were statistically significantly different from those of patients with chronic bronchitis and from those of healthy persons $(p<0.001)$. There was also a statistically significant difference between these parameters in patients with chronic bronchitis and in healthy persons at $10 \%$ vital capacity $(\mathrm{p}<0.001)$ but not at $90 \%$ vital capacity. The corresponding densitometric results for the upper and lower zones of the lungs in the three groups did not differ significantly.

The mean changes in densitometric parameters between $10 \%$ and $90 \%$ vital capacity for each study group are summarized in Table 3 . The mean changes in these densitometric parameters in the upper and the lower zones of the lungs were significantly greater in healthy persons than in patients who had emphysema or chronic bronchitis $(p<0.001)$, whereas the mean changes in patients who had emphysema and in those 
Table 3. Changes in Densitometric Parameters between $10 \%$ and $90 \%$ Vital Capacity.

\begin{tabular}{|c|c|c|c|c|}
\hline & \multirow{2}{*}{$\begin{array}{l}\text { Patients wilh } \\
\text { Emplysema } \\
(\mathrm{n}=20) \\
\text { Mean }+5 \mathrm{D}\end{array}$} & \multirow{2}{*}{$\begin{array}{l}\text { Patients with } \\
\text { Chronic Bronchitis } \\
(\mathrm{n}=20) \\
\text { Mean } \pm \mathrm{SD}\end{array}$} & \multicolumn{2}{|c|}{$\begin{array}{l}\text { Healthy Persons } \\
(n=20)\end{array}$} \\
\hline & & & Mean \pm SD & $\mathrm{p}^{*}$ \\
\hline \multicolumn{5}{|l|}{ Upper zones } \\
\hline Mean lung density & $23 \pm 14$ & $25 \pm 28$ & $73 \pm 40$ & $<.001$ \\
\hline Pixel index & $-14 \pm 7$ & $-10 \pm 9$ & $-32 \pm 21$ & $<.001$ \\
\hline Lowest 10 th percentile & $10 \pm 6$ & $10 \pm 11$ & $52 \pm 32$ & $<.001$ \\
\hline \multicolumn{5}{|l|}{ Lower zones } \\
\hline Mean lung density & $23 \pm 15$ & $24 \pm 19$ & $85 \pm 50$ & $<.001$ \\
\hline Pixel index & $-13 \pm 8$ & $-11 \pm 10$ & $-30 \pm 20$ & $<.001$ \\
\hline Lowest 10 th percentile & $11 \pm 8$ & $12 \pm 15$ & $46 \pm 29$ & $<.001$ \\
\hline
\end{tabular}

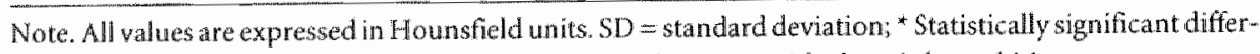
ence in comparison with patients, with emphysema and patients with chronic bronchitis.

who had chronic bronchitis did not differ significantly $(p>0.05)$. In no group were changes in densitometric parameters in the upper zones of the lungs significantly different from the corresponding densitometric parameters in the lower zones of the lungs.

Table 4 summarizes the results of the comparison between pulmonary function tests, densitometric parameters, and mean visual emphysema score. The best correlations were found for $\mathrm{FEV}_{1}$ and $\mathrm{K}_{\mathrm{CO}}$ and the three densitometric parameters at $10 \%$ vital capacity in the upper zones of the lungs. The correlations between $\mathrm{FEV}_{1}$ and the densitometric parameters were poor if the CT scan was made at $90 \%$ vital capacity, whereas the correlations between $\mathrm{K}_{\mathrm{CO}}$ and the densitometric parameters were similar at $10 \%$ and at $90 \%$ vital capacity. $K_{C O}$ had the highest correlation with the mean visual emphysema score.

The correlations between the mean visual emphysema score and the various densitometric parameters in upper and lower zones of the lungs are shown in Table 5. The best correlation was between the percentage of pixels with attenuation of less than $-910 \mathrm{HU}$ and the mean visual emphysema score in the upper zones of the lungs at $10 \%$ vital capacity $(r=0.69, p<0.001)$. Comparison of the correlation coefficients of the three densitometric parameters and the mean visual emphysema score revealed no statistically significant differences. In a comparison of correlation coefficients at $10 \%$ vital capacity and the corresponding correlation coefficients at $90 \%$ vital capacity, only the correlation coefficient between the pixel index and the mean visual emphysema score differed statistically significantly $(p<0.05)$; A correlation coefficient was greatest at $10 \%$ vital capacity. 
Table 4. Correlation of Densitometric Parameters and Mean Visual Emphysema Socre with Puhronary Function Tests in Whole Population.

\begin{tabular}{|c|c|c|c|c|c|c|}
\hline Pulmonary Function & Mean Lung & Pixel & & Lowest lot & & Mean Visual \\
\hline Test $\%$ predicted) & Density & Index & $p$ & $\begin{array}{l}\text { Percentile } \\
\text { (HU) }\end{array}$ & $\mathrm{p}$ & $\begin{array}{l}\text { Enplysemid } \\
\text { score }\end{array}$ \\
\hline
\end{tabular}

\section{Upper zones}

$10 \%$ vital capacity

$\begin{array}{lllllllll}- \text { FEV }_{3} & .56 & <.001 & -.66 & <.001 & .62 & <.001 & -.51 & <.001 \\ - \text { Total lung capacity } & .18 & & -.15 & & .13 & & -.28 & \\ -K_{c o} & .52 & <.001 & -.50 & <.001 & .47 & <.001 & -.69 & <.001\end{array}$

$90 \%$ vital capacity

- FEV, $\quad .15$

- Total lung capacity

.22

\begin{tabular}{|c|c|c|c|c|c|c|}
\hline & -.15 & & .29 & $<.05$ & -.51 & $<.001$ \\
\hline .01 & .19 & & .11 & & -.28 & \\
\hline$<.001$ & -40 & $<.001$ & .43 & $<.01$ & -.69 & $<.001$ \\
\hline
\end{tabular}

\section{Lower zones}

$10 \%$ vital capacity

\begin{tabular}{lcccccccc} 
- FEV & .49 & $<.001$ & -.59 & $<.001$ & .61 & $<.001$ & -.51 & $<.001$ \\
- Totall lung capacity & .08 & & -.12 & & .02 & & -.28 & \\
- Ko & .41 & $<.001$ & -.47 & $<.001$ & .43 & $<.001$ & -.69 & $<.001$ \\
$90 \%$ vital capacity & & & & & & & & \\
- FEV & .1 & & -.17 & & .36 & $<.001$ & -.51 & $<.001$ \\
- Total lung capacity & .22 & & .12 & & .11 & & .28 & \\
$-K_{6}$ & .39 & $<.001$ & -.34 & $<.01$ & .44 & $<.001$ & -.69 & $<.001$ \\
\hline
\end{tabular}

Table 5. Correlation between Mean Visual Emphysema Score and Densitometric Parameters.

\begin{tabular}{|c|c|c|c|}
\hline & $\begin{array}{l}\text { Mean Lung Density } \\
\text { (r) }\end{array}$ & $\begin{array}{l}\text { Pixel Index } \\
\text { (r) }\end{array}$ & $\begin{array}{l}\text { Lowest 10th } \\
\text { Percentile } \\
\text { (r) }\end{array}$ \\
\hline \multicolumn{4}{|l|}{ Upper zones } \\
\hline $10 \%$ vital capacity & -.60 & $.69 p<0.05$ & -.60 \\
\hline $90 \%$ vital a apacity & -.57 & $.54^{\prime}$ & -.62 \\
\hline \multicolumn{4}{|l|}{ Lower zones } \\
\hline $10 \%$ vital capaciry & -.40 & .47) $p<.05$ & -.47 \\
\hline $90 \%$ vital capacity & -.37 & $.32^{f}$ & -.50 \\
\hline
\end{tabular}

Note. All correllation coefficients are statistically significant: $p<.00 \mathrm{l}$. 


\section{DISCUSSION}

The present study confirms the value of spirometrically controlled CT densitometry in the quantitative assessment of lung density at selected levels of inspiration (5). In patients who had emphysema, regardless of the level of inspiration, all three densitometric parameters differed substantially from the corresponding values in patients who had chronic bronchitis and in healthy persons, whereas those in patients who had chronic bronchitis and in healthy persons were statistically significantly different only at 10\% vital capacity. The densitometric results in the upper and lower zones of the lungs were more or less identical.

CT densitometry gives objective and observer-independent information about diseases such as pullmonary emphysema and pulmonary fibrosis that affect lung density. Until now, three densitometric parameters have commonly been used: mean lung density (2); pixel index, which indicates overall percentage of lung involved with emphysema $(2,4,11)$; and the CT EMI number that defined the lowest 5 th percentile of the frequency distribution (3). These parameters are related to the physical density (in grams per cubic centimeter) of the scanned volume and are dependent not only on the structure of the lung tissue but also on the level of inflation and perfusion (12). Control of the level of inspiration during scanning, therefore, is important, especially when absolute lung density values will be used for diagnosis and follow-up. A spirometer connected to the CT scanner provides spirometrically triggered CT images. Reproducibility on the order of $5 \%$ can be achieved (5). This technique was used during our study and proved to be an easy applicable method that could be performed in all patients within 10 minutes. The computer program for CT data analysis, which includes contour tracking of both lungs, background deletion, and histogram analysis, permitted an instant quantitative evalution of the CT information.

In patients with emphysema, all the densitometric parameters that we used at $10 \%$ and $90 \%$ vital capacity differed statistically significantly from the corresponding values in healthy persons and in patients with chronic bronchitis, presumably due to alveolar destruction. In patients with chronic bronchitis and in healthy persons, these parameters differed at $10 \%$ vital capacity but not at $90 \%$ vital capacity, which may indicate that there was air trapping in patients with chronic bronchitis. This, combined with the differences in the changes in the densitometric parameters between $90 \%$ and $10 \%$ vital capacity in healthy persons and patients with chronic obstructive pulmonary disease, suggests that it may be possible to differentiate emphysema, chronic bronchitis, and healthy persons with the acquisition of two CT sections only: one section obtained at $90 \%$ vital capacity to distinguish emphysema from chronic bronchitis and healthy persons and a second, complementary section obtained at $10 \%$ vital capacity to distinguish chronic bronchitis from healthy persons. 
A reduction in $K_{C O}$, especially when coupled with hyperinflation and airway obstruction, is the best functional indication of emphysema. Diminished $\mathrm{K}_{\mathrm{CO}}$, however, is nonspecific and can be found in a wide variety of pulmonary disorders that affect the alveolocapillary membrane including interstitial lung disease and pulmonary vascular disorders (13). Furthermore, the level of $\mathrm{K}_{\mathrm{C}}$ may vary widely even in patients with litthe or no emphysema (14). As much as $30 \%$ of the lung may be involved with emphysema without any evidence of functional impairment (15). Airway obstruction is not invariably present in patients with mild emphysema (16). To examine the capabilities of quantitative CT in more pronounced emphysema, a more extreme cutoff for the diagnosis of emphysema was chosen in the present study than is generally recommended (6). The marked emphysematous changes in the selected patients who had emphysema were confirmed by quantitative CT and visual assessment of the thinsection CT scans.

Macroscopic emphysema is rarely evenly distributed throughout the lungs and predominates mostly in the upper zones of the lungs (11). We did not find, however, expected differences between densitometric parameters in the upper and the lower zones of the lungs in our patients who had emphysema. "This may have been caused by our relatively extreme inclusion criteria and by the selection of only patients with severe emphysema. The high mean visual emphysema score $(50 \pm 26)$ in this patient group, which is an assessment of the extent of emphysema in both lungs together, confirms this assumption. Our densitometric parameters were calculated from one section only, which may cause sampling error. The correlations between the mean visual emphysema score and the densitometric parameters in the upper zones of the lungs, however, were not statistically significantly different from the corresponding correlations in the lower zones of the lungs.

In the present study, three densitometric parameters - mean lung density, pixel index, and lowest 10th percentile of the frequency distribution - were compared with the mean visual emphysema score, which revealed no important differences. Gould et al (3) compared the extent of emphysema as scored pathologically in a lung section and the lowest 5 th percentile of the frequency distribution of EMI numbers in a corresponding CT section of 28 lung or lobe specimens after resection for a solitary tumor and found a statistically significant correlation between morphometric changes and the densitometric parameter. Our correllation between the lowest 10th percentile and the mean visual emphysema score was somewhat lower, but we callculated the mean visual emphysema score of both lungs together instead of the pathological score of one corresponding lung section. Müller et al (2) reported excellent correlation between the pathologic emphysema score and the percentage of lung with emphysema but a weak correlation with the mean lung density, which was, in their opinion ${ }_{\nu}$ of limited value because it did not detect regional localized areas of emphysema. They, however, obtained relatively thick $(1 \mathrm{~cm}) \mathrm{CT}$ sections at maximal inspiration after the infusion of a 
contrast medrum without controlling the level of inspiration during scanning. Gumey et al (11) found a good correlation in 59 smokers between the pixel index of the whole lungs obtained ar inspiration with the mean visual emphysema score. Their correlation is higher than ours, presumably because we correlated the pixel index of only one C.T section, which may have caused sampling errors.

The correlation of morphologic information such as CT data with the results of pulmonary function tests is done by many authors, and some of them claim high correlation $(4,17)$. We found a fair and statistically significant correlation between $K_{C o}$ and the mean visual emphysema score and moderate correlation between FEV $\mathrm{F}_{1}$ and the mean visual emphysema score. The correlation between $K_{c o}$ and the densitometric parameters at $90 \%$ and $10 \%$ vital capacity were lower than the correlation between $K_{C o}$ and the mean visual emphysema score, which may reflect the sampling error. The correlation between $\mathrm{FEV}_{1}$ and the densitometric parameters were more or less similar to the correlation between FEV 1 and the mean visual emphysema score when the CT section was obtained at $10 \%$ vital capacity and was significantly lower than the correlation between $\mathrm{FEV}$, and the mean visual emphysema score when the $\mathrm{CT}$ sections wete taken at $90 \%$ vital capacity.

In conclusion, spirometrically controlled CT lung densitometry is a well-tolerated examination that provides objective information about the density of the lung. There was a statistically significant correlation between objective and subjective parameters. Densitometric parameters were statistically significantly different in patients with em physena compared with patients with chronic bronchitis and healthy persons, irrespective of the level of inspiration, but differed only at $10 \%$ vital capacity in patients with chronic bronchitis compared with healthy persons. The mean change in densitometric parameters between $90 \%$ vital capacity and $10 \%$ vital capacity was large in healthy persons and was much less in patients with emphysema or chronic bronchitis. On the basis of these results, we presume that two sections obtained at the same level one at $90 \%$ vital capacity and one at $10 \%$ vital capacity - are suitable for the classification of persons into one of the three groups. Because of our relatively extreme selection ctiteria, however, we selected mainly patients with severe emphysema; our results, theretore, may be applicable only in this patient group. Further prospective studies are needed to evaluate the sensitivity and specificity of C T lung densitometry in the differential diagnosis of emphysema or chronic bronchitis.

\section{REFERENCES}

1. Miller RR, Muller NL, Veda1S, Morrison NJ, Staples CA. Limitations of computed tomography in the asisesment of emplysena. Am Rev Respir Dis 1989; 1.39:980-983.

2. Muller NL. Staples C.A. Miller RR, Abbond RT. "Densiby Mask": an objective method to quantitate emphysena using computed tomography. Chest 1988; 94:782-787. 
3. Gould GA, MacNee W, McLean A, et al. CT measurements of lung density in life can quantitate distal airspace enlargement: an essential defining feature of human emphysena: Am Rev Respir Dis 1988; $137: 380-392$.

4. Kinsella M, Müller NL, Abboud RT, Morrison NJ, DyBuncio A. Quantitation of emphysema by computed tomography using a "density mask" program and correlation with pulmonary function tests. Chest 1990;97:315-321.

5. Kalender WA, Rienmuller R, Seissler W, Behr J, Welke M, Fichte H. Measurement of pulmonary parenchymal attenuation: use of spirometric gating with quantitative CT. Radiology 1990; 175:265-268.

6. Openbrier DR, Irwin MM, Rogers RM, et al. Nutritional status and lung function in patients with enphysema and chronic bronchitis. Chest 1983;83:17-22.

7. American Thoracic Society. Chronic bronchitis, asthma and pulmonary emphysenat a statement by the Committee on Diagnostic Standards for Nontuberculous Respiratory Diseases. Am Rev Rev Respir Dis 1962; 85:762-767.

8. Quanjer PH. Standardized lung function testing. Bull Eur Physiopathol Respir 1983; 19:7-44.

9. Sakai F, Gamsu G, Im JG, Ray CS. Pulmonary function abnormalities in patients with CT-determined emphysema. J Comput Assist Tomogr 1987; 11:963-968.

10. Efron $B$. The Jackknife, the bootstrap and other resampling plans: regional conference series in applied mathematics. $1982 ; 38: 27-36$.

11. Gurney IW, Jones KK, Robbins RA, et al. Regional distribution of emphysema: correlation of highresolution CT with pulmonary function tests in unselected smokers. Radiology 1992; 183:457-463

12. Rosenblum $L$, Mauceri $R A$, Wellenstein $D E$, et al, Density patterns in the normal lung as determined by computed tomography. Radiology 1980; 137:409-416.

13. Klein IS, Gamsu G, Webb WR, Golden /A. Müller NL. High-resolution CT diagnosis of emphysemm in symptomatic patients with normal chest radiographs and isolated low diffusing capacity, Radiology $1992 ; 182: 817-821$.

14. Thurlbeck WM, Henderson JA, Fraser RG, Bates DV. Chronic obstructive lung disease: a comparison between dinical, roentgenologic, functional criteria in chronic bronchitis, asthma and bronchiectasis. Medicine 1970; 49:81-145.

15. Pratt PC. Role of conventional chest radiography in diagnosis and exclusion of emphyserna. Am I Med 1987; 82:998-1006.

16. Gelb AF, Gold WM, Wright RR, Bruch HR, Nadel JA. Physiologic diagnosis of subclinical emphysema. Am Rev Respir Dis 1973; 107:50-63.

17. Biernacki W, Gould GA, Whyte KF. Flenley DC. Pulmonary hemodynamics, gas exchange, and the severity of emphysema as assessed by quantiatwe CT scan in chronic bronchitis and emplyysema. Am Rev Respir Dis 1989; 139:1509-1515. 

CHAPTER 8

Emphysema and airflow limitation in patients with advanced chronic obstructive pulmonary disease:

a CT study

Rob J.S. Lamers

Emiel F.M. Wouters

Gerrit I. Kemerink

Jos M.A. van Engelshoven 


\section{ABSTRACT}

Purpose: To assess prospectively the association of emphysema with airflow limitation in patients with chronic obstructive pulmonary disease (COPD), and to study whether the diffusing capacity for carbon monoxide $\left(\mathrm{DL}_{\mathrm{CO}}\right)$ can be used as an indicator for emphysema.

Materials and methods: One hundred patients with COPD participated in this study. The population was primarily subdivided into three groups based on forced expiratory volume in one second $\left(\mathrm{FEV}_{1}\right) \%$ predicted results: stage I COPD is $\mathrm{FEV}_{1} \geq 50 \%$ predicted $(n=27)$; stage II COPD is $\mathrm{FEV}_{1} 35$ to $49 \%$ predicted $(n=32)$; and stage III COPD is $\mathbb{E E V} V_{1}<35 \%$ predicted $(\mathrm{n}=41)$. Secondly the population was subdivided based on FEV 1 results: advanced COPD is FEV $<1 \mathrm{~L}(\mathrm{n}=50)$, and $\mathrm{FEV}_{1} \geq 1 \mathrm{~L}(\mathrm{n}=$ 50). Five high-resolution CT (HRCT) scans of the lungs were obtained for visual assessment of emphysema, and subsequently 2 spirometrically controlled CT scans at $90 \%$ and $10 \%$ of the inspiratory vital capacity for objective assessment of lung density. HRCT scores and CT lung densitometry results were correlated with $D L_{C o}$ results.

Results: A low prevalence (15\%) of CT-scored emphysema was observed in patients who had stage I COPD and a high prevalence $(68 \%)$ of CT-scored emphysema in patients having stage III COPD. Lung density parameters increased at both levels of inspiration with increasing $\mathrm{FEV}_{1} \%$ predicted values. Attenuation values were significantly higher in patients with stage I COPD than in patients with stage III COPD $(p<0.0001)$. A high prevallence $(93 \%)$ of CT-scored emphysema was observed in patients with $\mathrm{DL}_{\mathrm{CO}}<50 \%$ predicted and a low prevalence $(19 \%)$ in patients with $\mathrm{DL}$ CO $\geq 50 \%$ predicted. There was significant correlation between $\mathrm{DL}_{\mathrm{CO}} \%$ predicted and the CT emphysema score $(r=-0.70, p<0.0001)$ and the mean lung density at $90 \%$ vital capacity $(r=0.72, p<0.0001)$. Also in patients with $F E V_{1}$ values $<1 \mathrm{~L}$, the correlation between $D L_{C O} \%$ predicted and the CT emphysema score was $-0.73(\mathrm{p}<0.0001)$, and lung densitometry $0.72(p<0.0001)$. However, in patients with $D L_{C O}<50 \%$ predicted corresponding correlations were considerably lower $(r=-0.47, p<0.05 ; \mathrm{r}=$ $0.26, p<0.005$ respectively).

Conclusion: Results suggest a distinct presence and degree of emphysema in patients with advanced COPD. DL $\mathrm{CO}_{\mathrm{O}}$ is a useful test to separate patients with COPD with emphysema from patients with COPD without emphysema. However, $\mathrm{DL}_{\mathrm{CO}}$ measurements cannot be relied on for quantitative assessment of severity and extent of emphysema. DLCO measurements are therefore valuable to select patients for radiologic emphysema assessment. 


\section{INTRODUCTION}

Chronic obstructive pulmonary disease (COPD) is defined as a disease state characterized by the presence of airflow limitation due to emphysema or chronic bronchitis (1). Emphysema is defined as the abnomal permanent enlargement of airspaces distal to the terminal bronchioles, accompanied by destruction of their walls without obvious fibrosis (1). Functional hallmarks of emphysema are decreased airflow at spirometry and decreased carbon monoxide single-breath diffusing capacity (DL $\mathrm{CO}_{\mathrm{CO}}$ ) due to parenchymal destruction. The separate contribution of emphysema and chronic bronchitis in causing airflow limitation in COPD is difficult to assess because the mechanical principles underlying airflow obstruction are interrelated in a complex way. Some researchers ascribe a major role for emphysema in causing airflow limitation due to a loss of lung elastic recoil $(2,3)$. Others suggest that emphysema is only a relatively minor determinant of pulmonary function in advanced $\operatorname{COPD}(4,5)$, and that the decrease in expiratory flow in patients with COPD is due to an inflammatory process in the small conducting airways $(5-10)$. The $\mathrm{DL}_{\mathrm{CO}}$ is supposed to be decreased in proportion to the severity of emphysema (11-13), but is also considered to be a nonspecific indicator of impaired gas transfer, in particular, not suitable to detect mild emphysema (14).

To date computed tomography (CT) is considered the most accurate imaging method for diagnosing emphysema in vivo (15-17) and CT findings in pulmonary emphysema correlate closely to pathologic findings. The overall extent of emphysema can be assessed subjectively by visual inspection of $\mathrm{CT}$ images, or objectively, by use of a computer program analyzing attenuation values. Drawback of visual scoring techniques is that they depend on the experience of the observer and an $10 \%$ intraobserver and interobserver variability has been reported (18). Lung density, however, is considerably influenced by the level of inspiration at which the CT images are obtained and control and monitoring of the degree of inspiration during scanning is important to obtain reproducible results. We reported a reproducibility of mean lung density measurements within $3 \mathrm{HU}$ at $90 \%$ vital capacity and within $11 \mathrm{HU}$ at $10 \%$ vital capacity using this respiratory triggered technique (19). Reproducibility of lung density measurements was not influenced by the presence of respiratory insufficiency (19).

Gelb et al throw doubt upon the value of the $\mathrm{DL}_{C}$ to detect significant emplyysema in patients with advanced COPD $(4,5)$. They compared lung function and $\mathrm{CT}$ grading concerning pulmonary emphysema in patients with COPD and could not demonstrate a relation between airflow limitation ( $F E V_{\mathbb{I}}$ ) and emphysena ( $C T$ emphysema score) and even between $\mathrm{DL}_{\mathrm{CO}} \%$ predicted and radiologically assessment of emphysema in patients with advanced COPD.

The purpose of our study was to assess the contribution of emphysema in causing airflow limitation in patients with advanced COPD, and to study the debated point 
whether DLCO can be used as an indicator for emphysema in advanced COPD. We used high resolution $\mathrm{CT}$ of the lungs, in combination with $\mathrm{CT}$ lung densitometry, as the gold standard for the diagnosis of emphysema and for assessment of its severity.

\section{MATERIALS AND METHODS}

A group of 100 patients $(71$ men, 29 women; mean age 63 years; age range, 39-83 years) with COPD as defined by the criteria of the American Thoracic Society (20) was entered into this prospective study. All patients had respiratory symptoms (chronic productive cough and/or shortness of breath during exertion or at rest) as well as a forced expiratory volume in one second $\left(\mathrm{FEV}_{1}\right)$ of less than $70 \%$ of the reference value and a difference between prebronchodilator and postbronchodilator values of FEV not exceeding $10 \%$ of predicted. CT scans were performed in a clinically stable period, free from exacerbation. Informed consent was obtained from all the patients. The study protocol was approved by the ethics committee of the hospital.

\section{Pulmonary function studies}

Lung function measurements were obtained by means of a wet spirometer (Gould Pulmonet III; Sensor Medics, Bilthoven, the Netherlands). FEV 1 was expressed as a percentage of the reference values (21). Lung volumes were measured with wholebody plethysmography and expressed as a percentage of the reference values (21). The diffusing capacity for carbon monoxide ( $\mathrm{DL}_{\mathrm{CO}}$ ) was measured using the single-breath technique (MasterLab; Jaeger, Würzburg, Germany). Transfer factor for carbon monoxide $\left(\mathrm{K}_{\mathrm{CO}}\right)$ was calculated from the ratio of $\mathrm{DL}_{\mathrm{CO}}$ to alveolar volume. Lung function tests were performed by trained technicians according the ERS guidelines (22).

\section{CTemphysema score}

From each person, five thin-section computed tomography (HRCT) scans of the lungs (Somatom Plus; Siemens, Erlangen, Germany) were obtained at full inspiration with the patient in supine position: two scans of the upper zones at $3 \mathrm{~cm}$ and $6 \mathrm{~cm}$ above the carina, two scans of the lower zones at $3 \mathrm{~cm}$ and $6 \mathrm{~cm}$ below the carina, and one at the level of the carina. Scanning parameters were 1.0 -mm collimation, $137 \mathrm{kVp}$, $220 \mathrm{~mA}, 1.0$ second scanning time, and a high-resolution reconstruction algorithm. Hardcopy images were photographed using a window setting appropriate for visualization of lung parenchyma (level: $-800 \mathrm{HU}$; width: 1,600 HU). Each slice was scored by two radiologists (R.J.S.L., J.M.A. v. E.) separately, without awareness of clinical findings, to a visual CT emphysema score according to the method of Sakai, which is 
based on the assessment of 2 aspects of emphysema: severity and extent (23). Severity was graded on a 4-point scale: 0 , no emphysema; 1 , low attenuation areas $<5 \mathrm{~mm}$ in diameter; 2 , circumscribed low attenuation areas $>5 \mathrm{~mm}$ in addition to those $<5 \mathrm{~mm}$; 3 , diffuse low attenuation areas without normal intervening lung. The extent of emphysema was determined using a 4-point scale: $1,<25 \%$ of the lung parenchyma involved; 2,25 to $50 \%$ involvement; 3,50 to $75 \%$ involvement; $4,>75 \%$ involvement. For each hemislice, the score for severity was multiplied by the score for the extent. The scores of the 5 CT slices were summed to yield a CT emphysema score that could range from zero (no emphysema) to 120 (severe emphysema). The mean CT emphysema score of both observers was calculated for the purpose of this study. Patients were considered to have no or trivial emphysema if the CT emphysema score was $<30$, and to have significant emphysema if the CT emphysema score was $\geq 60$.

\section{CT lung densitometry}

Additionally during the same session, CT scans of the lungs were obtained in supine position for quantitative assessment of lung density patterns. The following scanning parameters were employed: $1.0-\mathrm{mm}$ collimation, $137 \mathrm{kVp}, 220 \mathrm{~mA}$, and 1.0 second scanning time. Scans were reconstructed in the soft-detailed resolution mode. The full width at half maximum of the point spread function of this reconstruction filter is 1.86 $\mathrm{mm}$; the sample volume $3.92 \mathrm{~mm}^{3}(24)$. No intravenous contrast material was administered. In order to control the level of inspiration during scanning, the patient was asked to breath through a pocket spirometer connected with the CT scanner (25). At user-selected levels of inspiration airflow was interrupted mechanically by a closing valve in the spirometer at what time a trigger signal was generated to start the scanner. The level of inspiration was kept constant for the duration of the CT scan. CT scans were obtained at $5 \mathrm{~cm}$ above the level of the carina and at $90 \%$ and $10 \%$ of the inspiratory vital capacity. Evaluation of the CT images was done on a SUN Sparc 1+ graphical workstation (SUN Microsystems, Mountain View, Calif). Both lungs were segmented automatically at a CT level of $-200 \mathrm{HU}$ using pixel tracing (26). Subsequently, the segmented areas were slightly eroded ( 2 pixels) to remove the circumference that partially corresponded to the thoracic wall. The histograms of both lungs were analyzed together. The mean lung density and the cut-off point of the frequency distribution that defines the lowest 10 th percentile were calculated. Both lung density parameters have been often used for tissue characterization (27-30). CT during $90 \%$ vital capacity is useful to assess the extent of emphysema, whereas CT during $10 \%$ vital capacity is helpful in assessing degrees of air trapping $(31,32)$. 


\section{Statistical analysı̈:}

Interobserver agreement of visual assessment of emphysema was tested using Spearman's rank correlation coefficient. Statistical comparison between groups of patients was performed with the Student's $t$ test. The results of pulmonary function tests were correlated with the CT emphysema score using Spearman's rank correlation coefficient, and with CT lung densitometry results with the Pearson correlation coefficient. A probability of less than 0.05 was considered to indicate a statistically significant difference in all analyses.

\section{RESULTS}

The results of pulmonary function tests, visual assessment of CT scan images, and CT densitometry of the lung are summarized in Table 1. Patients suffered from moderate to severe airflow limitation. In 50 patients the $\mathrm{FEV}_{1}$ was $<1 \mathrm{~L}$. Further there was a wide spectrum of $\mathrm{DL}_{\mathrm{CO}} \%$ predicted and $\mathrm{K}_{\mathrm{CO}} \%$ predicted values ranging from normal to greatly reduced. In 19 patients we were not able to perform a proper diffusing capacity measurement; of these, 17 patients with a $F E V_{1}<1 \mathrm{~L}$, and 2 patients with a $F E V_{1} \geq 1 \mathrm{~L}$. There was good interobserver agreement for the CT emphysema score $(r=0.82, p<$ 0.001). The mean CT emphysema score in patients with a FEV $<1 \mathrm{~L}$ was 43 (range 0 117) and significantly higher $(\mathrm{p}<0.05)$ than the mean CT emphysema score of patients with a $E E V_{1} \geq 1 \mathrm{~L}(27$, range $0-102)$. In 18 patients $(36 \%)$ with a $F E V_{1}<1 L$ and in 33 patients $(66 \%)$ with $\mathrm{FEV}_{1} \geq 1 \mathrm{~L}$, the $\mathrm{CT}$ emphysema score was $<30$, indicating no or trivial emphysema. In 17 patients $\left(34 \%\right.$ ) with a $\mathrm{FEV}_{\mathbb{1}}<1 \mathrm{~L}$ and in 9 patients (18\%) with a FEV $1 \geq 1 \mathrm{~L}$, the $\mathrm{CT}$ emphysema was $\geq 60$, indicating significant emphysema. The mean CT emphysema score of the 19 patients in which we were not successful to obtain a reliable diffusing capacity measurement was 45 (range, 0-102).

According to the American Thoracic Society statement on interpretation of lung function tests, the population was subdivided into three groups (1): stage $\mathbb{C}$ COPD is $50 \leq$ $\mathrm{FEV}_{1}<70 \%$ predicted; stage II COPD is $35 \leq \mathrm{FEV}_{1}<50 \%$ predicted; and stage III COPD is FEV $<35 \%$ predicted (Table 2). The mean CT emphysema score of patients with stage 1 COPD was 12 (range, 0-60) and significantly lower than the mean CT emphysema score (37; range 0-115) of patients with stage II COPD ( $<<0.005$ ), and significantly lower than the mean CT emphysema score (48; range, 0-117) of patients with stage III COPD ( $p<0.0001$ ). The relation between $\mathrm{FEV}, \%$ predicted and the CT empthysema score is described in Figure $1\left(r=-0.46_{n} p<0.0001\right)$. In 23 of the 27 patients $(85 \%)$ with stage I COPD the CT emphysena score was $<30$. Furthermore, in 19 of the 41 patients (47\%) with stage III COPD, the CT emphysema score was $\geq 60$. 
Table 1. Pulmonary Function, CT Emphysema Score, and Lung Attenuation Data of 100 Patients with COPD.

\begin{tabular}{|c|c|c|c|c|}
\hline Parameter & Mean & $\pm S D$ & Range & \\
\hline $\mathrm{FEW}_{\mathrm{z}}^{\mathrm{F}} \%$ predicted & 40 & \pm 14 & 17 & 70 \\
\hline $\mathrm{FEW}_{\mathrm{k}}, \mathrm{L}$ & 1.09 & $\pm \quad 0.41$ & 0.41 & 236 \\
\hline FVC, \% predicted & 88 & \pm 17 & 45 & 122 \\
\hline$F E V / \mathrm{FVC}_{3}$ & 46 & \pm 1.5 & 23 & 111 \\
\hline TLC, \% predicted & 124 & \pm 18 & 82 & 185 \\
\hline DL,$\%$ predicted & 61 & \pm 23 & 18 & 115 \\
\hline $\mathbb{K}_{w, \%} \%$ predicted & 64 & \pm 27 & 17 & 136 \\
\hline C.T emphysema score & 35 & \pm 32 & 0 & 117 \\
\hline MLD $90 \% \mathrm{VC}, \mathrm{HU}$ & -894 & $\# 32$ & -785 & $-\quad-957$ \\
\hline MLD $10 \%$ VC, HU & -856 & \pm 47 & -733 & $-\quad-940$ \\
\hline LTP $90 \%$ VC, $\mathrm{HU}$ & -962 & \pm 29 & -870 & --1004 \\
\hline $\mathrm{LTP} 10 \% \vee \mathrm{CC}_{3}, \mathrm{HU}$ & -942 & \pm 42 & -797 & --1005 \\
\hline
\end{tabular}

Note. $M L D=$ mean lung density and LTP $=$ lowest 10 th percentile are expressed in Hounsfield units. SD $=$ standard deviation.

Table 2. Prevalence of Airflow Limitation Stratified for the CT Emphysema Score in 100 Patients with COPD.

\begin{tabular}{|c|c|c|c|c|c|}
\hline & \multirow{2}{*}{$\begin{array}{l}\text { Patients } \\
\text { (n) }\end{array}$} & \multicolumn{4}{|c|}{ CT Emphysema Score } \\
\hline & & $<30$ & $30-59$ & $60-89$ & $\geq 90$ \\
\hline Stage I COPD & 27 & $23(85)$ & $3(11)$ & $1(4)$ & $0(0)$ \\
\hline Stage II COPD & 32 & $16(50)$ & $8(25)$ & $4(13)$ & $4(13)$ \\
\hline \multirow[t]{2}{*}{ Stage III COPD } & 41 & $13(32)$ & $9(22)$ & $1.5(37)$ & $4(10)$ \\
\hline & & 52 & 20 & 20 & 8 \\
\hline
\end{tabular}

Note * CT emphysema score $<30=$ no or trivial emphysema; 30 to $59=$ mild emphysema; 60 to $89=$ mod erate emphysema; $\geq 90=$ severe enphysema. Numbers in parenthesis are percentages. Percentages may not add to 100 because of rounding.

Lung density parameters increased at both levels of inspiration with increasing $\mathrm{FEV}_{1}$ $\%$ predicted values (Table 3 ). Densitometric parameters in patients with stage I COPD were statistically significantly higher than those from patients with stage III COPD ( $\mathrm{p}$ $<0.0001$ ), and also from those with stage II COPD at both levels of inspiration ( $p<$ 0.005 ). Densitometric results between patients with stage II and stage III COPD did not differ significantly. The change between the densitometric data obtained at $90 \%$ vital capacity and at $10 \%$ vital capacity was significantly higher in patients with stage I COPD than the corresponding change between the densitometric data in patients with stage III COPD $(\mathrm{p}<0.001)$. 
$\because=-40,0<0.000$

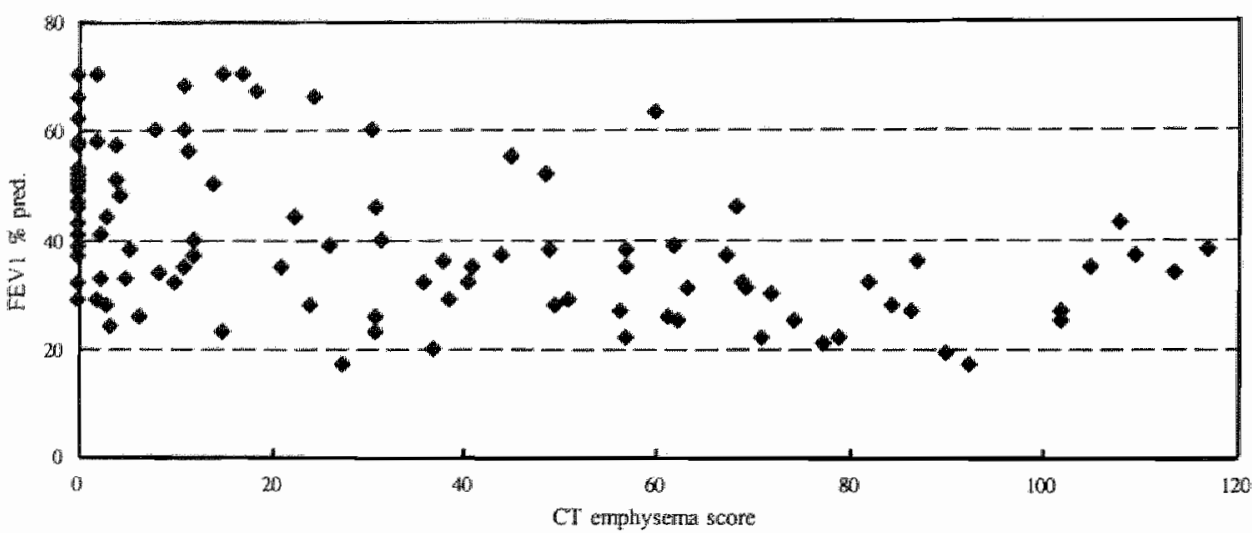

Figure 1. Relation between FEVI of predicted and the CrT emphysema score in 100 patients with irreversible ärDow limitation.

Table 3. Densitometric Results at $90 \%$ and $10 \%$ Vital Capacity of 100 Patients with COPD.

\begin{tabular}{|c|c|c|c|c|c|}
\hline \multirow[b]{2}{*}{ Vital Capacity $(\%)$} & \multirow{2}{*}{$\begin{array}{l}\text { Stage I COPD } \\
(n=27) \\
\text { Mean } \pm S D\end{array}$} & \multicolumn{2}{|c|}{$\begin{array}{l}\text { Stage II COPD } \\
(\mathrm{n}=32)\end{array}$} & \multicolumn{2}{|c|}{$\begin{array}{l}\text { Stage III COPD } \\
(n=41)\end{array}$} \\
\hline & & Mean + SD & $p^{*}$ & Mean $\pm S D$ & $p+$ \\
\hline \multicolumn{6}{|c|}{ Mean lung density (HU) } \\
\hline 90 & $-873 \pm 29$ & $-897 \pm 32$ & $<.005$ & $-905 \pm 23$ & $<.0001$ \\
\hline 10 & $-819 \pm 34$ & $-860 \pm 47$ & $<.000 \mathrm{I}$ & $-877 \pm 38$ & $<.0001$ \\
\hline \multicolumn{6}{|c|}{ Lowest 10th perc. (HU) } \\
\hline 90 & $-941 \pm 21$ & $-965 \pm 29$ & $<0.005$ & $-974 \pm 21$ & $<.0001$ \\
\hline 10 & $-908 \pm 37$ & $-947 \pm 45$ & $<.0005$ & $-963 \pm 33$ & $<.0001$ \\
\hline
\end{tabular}

Note. Clnumbers aregiven as means 150 , and expressed in Hounsfield Units (HU): Patients with stage $11 \mathrm{COPD}$ versus those with stage III COPD; + Patients with stage III COPD versus those with stage ICOPD.

Table 4. Densitometric Results $90 \%$ and $10 \%$ Vital Capacity of 100 Patients with COPD Stratified for the CT Emphysema Score.

\begin{tabular}{|c|c|c|c|c|}
\hline \multirow[b]{2}{*}{ Vital capacity $(\%)$} & \multicolumn{4}{|c|}{ CT Emphysema Score* } \\
\hline & $\begin{array}{l}<30 \\
(n=52)\end{array}$ & $\begin{array}{l}30-59 \\
(n=20)\end{array}$ & $\begin{array}{l}60-89 \\
(n=20)\end{array}$ & $\begin{array}{l}\geq 90 \\
(n=8)\end{array}$ \\
\hline \multicolumn{5}{|c|}{ Mean lung density (HU) } \\
\hline 90 & $-871 \pm 23$ & $-907 \pm 13$ & $-921 \pm 20$ & $-939 \pm 10$ \\
\hline 10 & $-821 \pm 38$ & $-877 \pm 21$ & $.901 \pm 25$ & $-919 \pm 12$ \\
\hline \multicolumn{5}{|c|}{ Lowest 10th perc. (HU) } \\
\hline 90 & $-940 \pm 22$ & $-978 \pm 11$ & $-989 \pm 8$ & $-997 \pm 5$ \\
\hline 10 & $-909 \pm 37$ & $-967 \pm 16$ & $-986 \pm 12$ & $-996 \pm 7$ \\
\hline
\end{tabular}

Note. ${ }^{*}<30=$ no or trivial emphysema; 30 to $59=$ mild emphysema; 60 to $89=$ moderate emphysema; $\geq 90=$ severe emphysema. CT numbers are given as means $\pm S D$. 


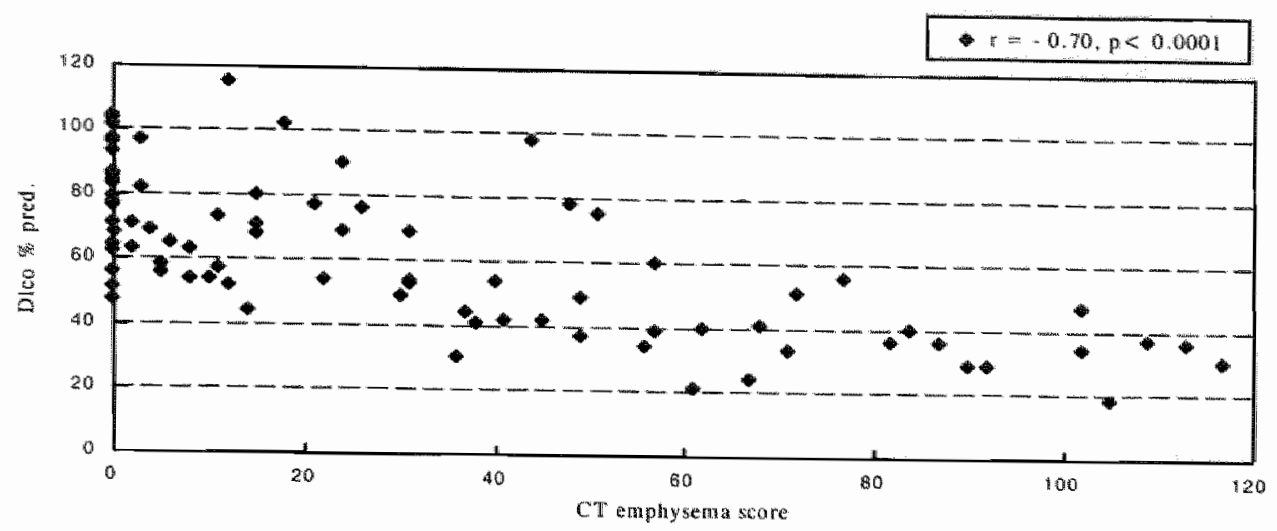

Figure 2. Relation between D 1 co \% predicted and the CT emphysema score in 81 patients with irteversible airtlow limitation.

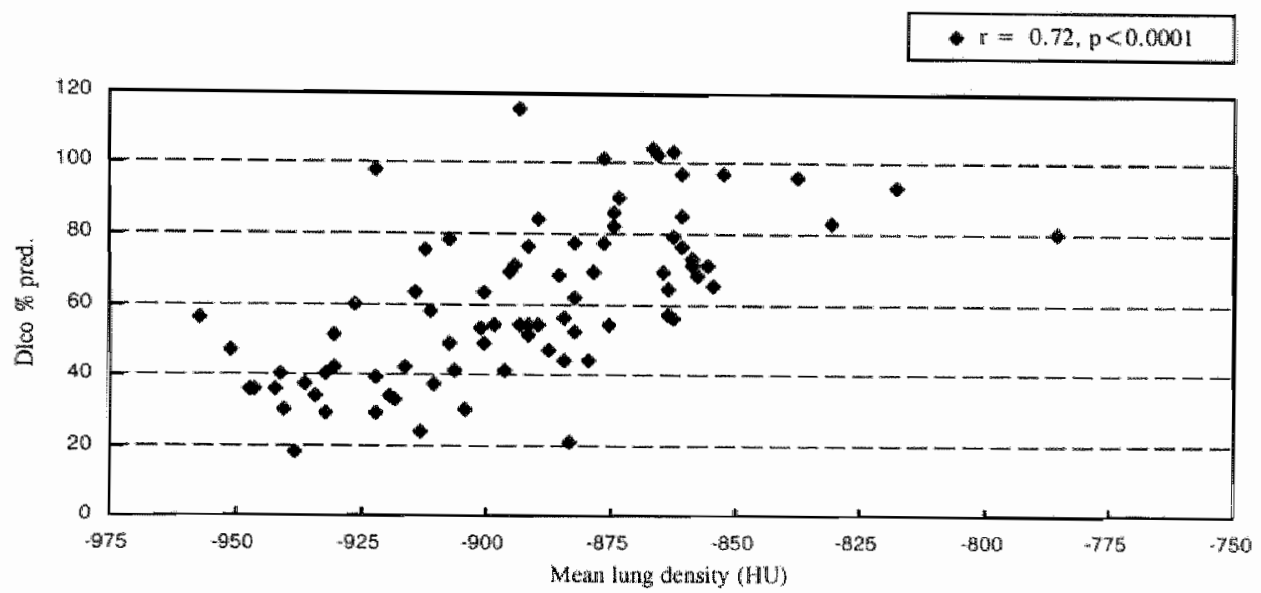

Figure 3. Relation between DIco \% predicted and the mean lung densiry at $90 \%$ vital capacily in 81 patients with irreversible airflow limitation.

For further analysis the group of patients with COPD was subdivided on the basis of the CT emphysema score into four categories; no or trivial emphysema ( $\mathrm{CT}^{3}$ grade < 30), mild emphysema (CT grade 30-59), moderate emphysema (CT grade 60-89), and severe emphysema (CT grade $\geq 90$ ) (Table 4 ). With increasing extent of emphysema, as indicated by the $\mathrm{CT}$ emphysema score, attenuation values decreased at both levels of inspiration. Lung density parameters were significantly lower in the group with severe emphysema than in the group with no or trivial emphysema $(\mathrm{p}<0.001)$. The change between the mean lung density at $90 \%$ vital capacity and at $10 \%$ vital capacity in the group with severe emphysema was $20 \pm 9 \mathrm{HU}$, and for the lowest 10th percentile 


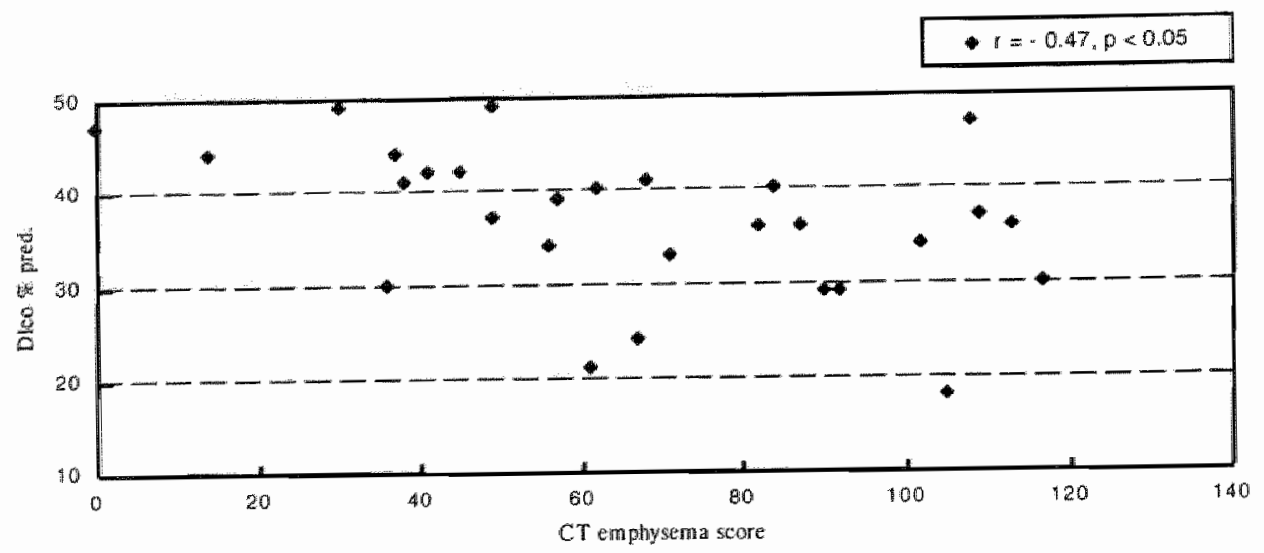

Figure 4. Relation between D 1 co \% predicted and Cr emphysema score in 28 patients with COPD and D 1 co \% predicled less than 50 .

$r=0.26,0<0.005$

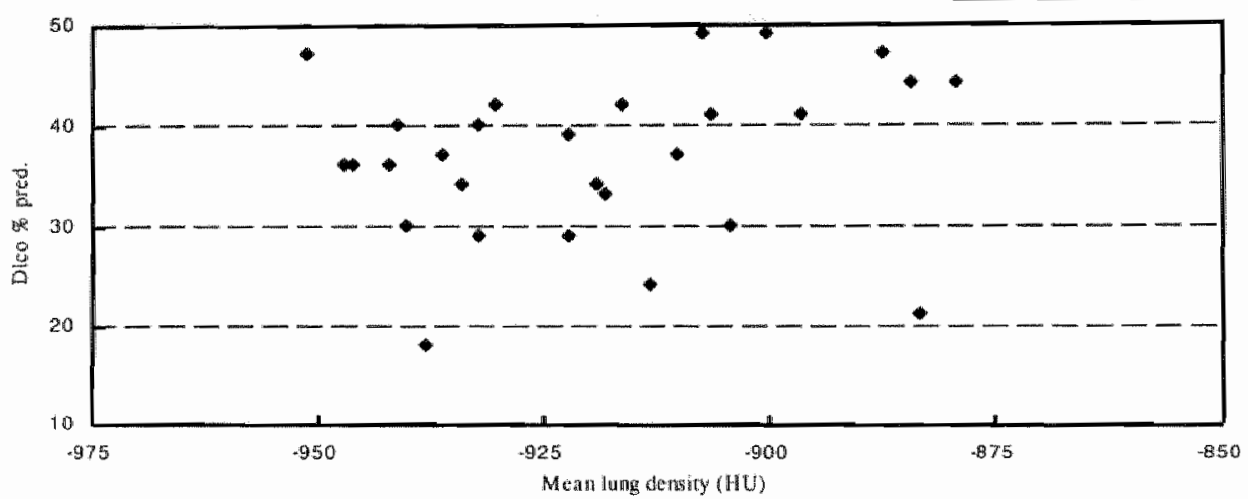

Figure 5. Relation between DIco \% predicted and mean lung density at $90 \%$ vital capacity in 28 patients with COPD and Dloo sededed less than 50 .

$1 \pm 3 \mathrm{HU}$. In the group with no or trivial emphysema these changes between the densitometric data obtained at $90 \%$ vital capacity and $10 \%$ vital capacity were $50 \pm 21 \mathrm{HU}$ and $31 \pm 17 \mathrm{HU}$ respectively. Changes between the densitometric data were significantly smaller in the group with severe emphysema than in the group with no or trivial emphysema $(p<0.005)$.

The relationship between $\mathrm{DL}_{\mathrm{CO}} \%$ predicted and the $\mathrm{CT}$ emphysema score in patients with COPD is shown in Figure $2(\mathrm{r}=-0.70, \mathrm{p}<0.0001)$, and between $\mathrm{DL}_{\mathrm{CO}} \%$ predicted and the mean lung density at $90 \%$ vital capacity in Figure $3(r=0.72, p<$ 0.0001 ). In 28 patients ( $35 \%$ ) the $\mathrm{DL}_{\mathrm{CO}}$ was $<50 \%$ predicted, and in 53 patients $(65 \%)$ 
- $r \neq-0.3 . p<0.0001$

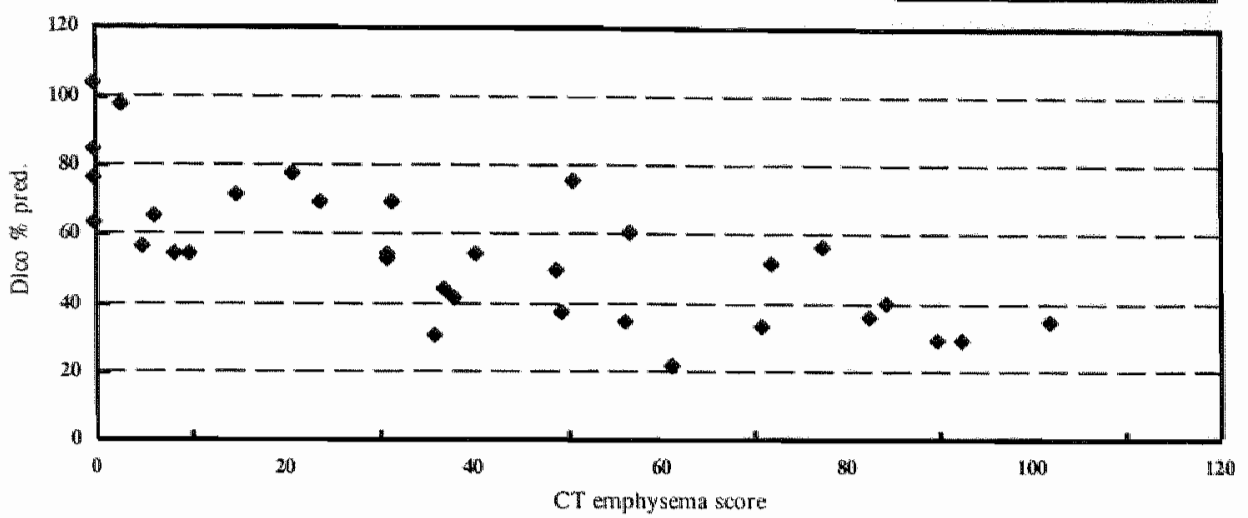

Figure 6. Rellation betweer DIco \% predicted and CT emphysema score in 33 patients with COPD and FEV I less than 1L.

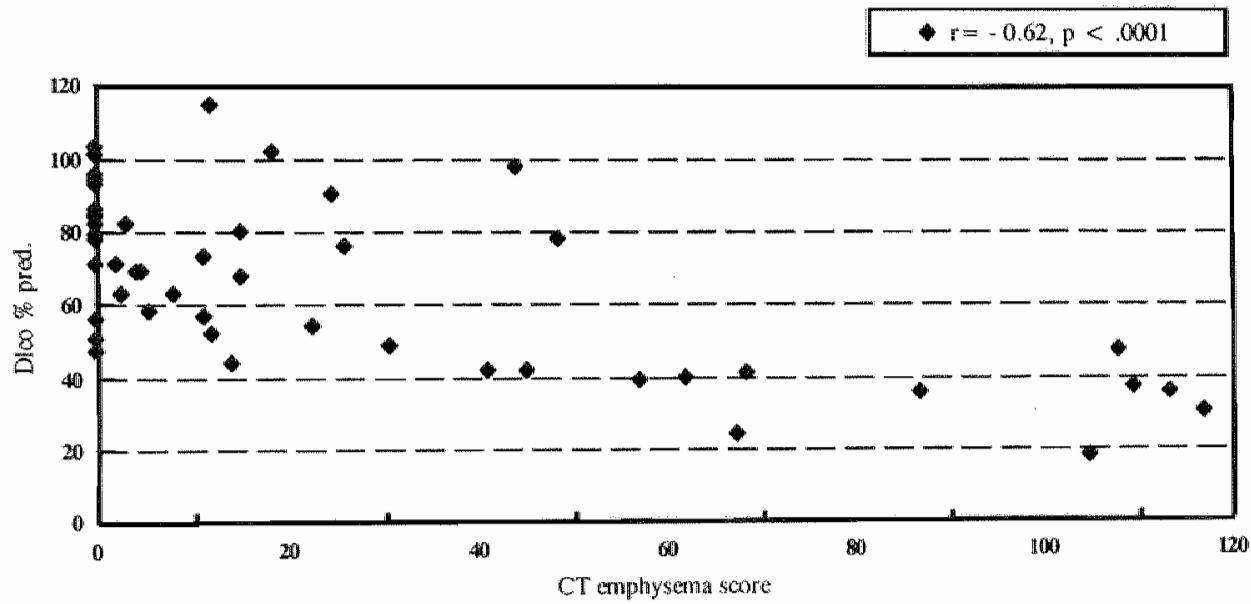

Figure 7. Relation between D 1co\% predicted and CT emphysema score in 48 patients with COPD and FEVI equal to or more than $\mathrm{LL}$.

the $\mathrm{DL}_{\mathrm{CO}}$ was $\geq 50 \%$ predicted. In patients with $\mathrm{DL}_{\mathrm{CO}}<50 \%$ predicted, the relation between $\mathrm{DL}_{\mathrm{CO}} \%$ predicted and the CT emphysema score is described in Figure $4(\mathrm{r}=$ $-0.47, \mathrm{p}<0.05)$, and between $\mathrm{DL}$ CO \% predicted and the mean lung density at $90 \%$ vital capacity in Figure $5(r=0.26, \mathbb{P}<0.005)$. In patients with $D_{C_{C O}} \geq 50 \%$ predicted, a significant correlation was found between $\mathrm{DL}_{\mathrm{CO}} \%$ predicted and the CT emphysema score $(\mathrm{r}=-0.34, \mathrm{p}<0.05)$, and between $\mathrm{DL}_{\mathrm{CO}} \%$ predicted and the mean lung density at $90 \%$ vital capacity $(r=0.46, p<0.001)$. In 26 of 28 patients $(93 \%)$ with $\mathrm{DL}_{\mathrm{CO}}<50$ 


\section{$r=0.72, p<0.0001$}

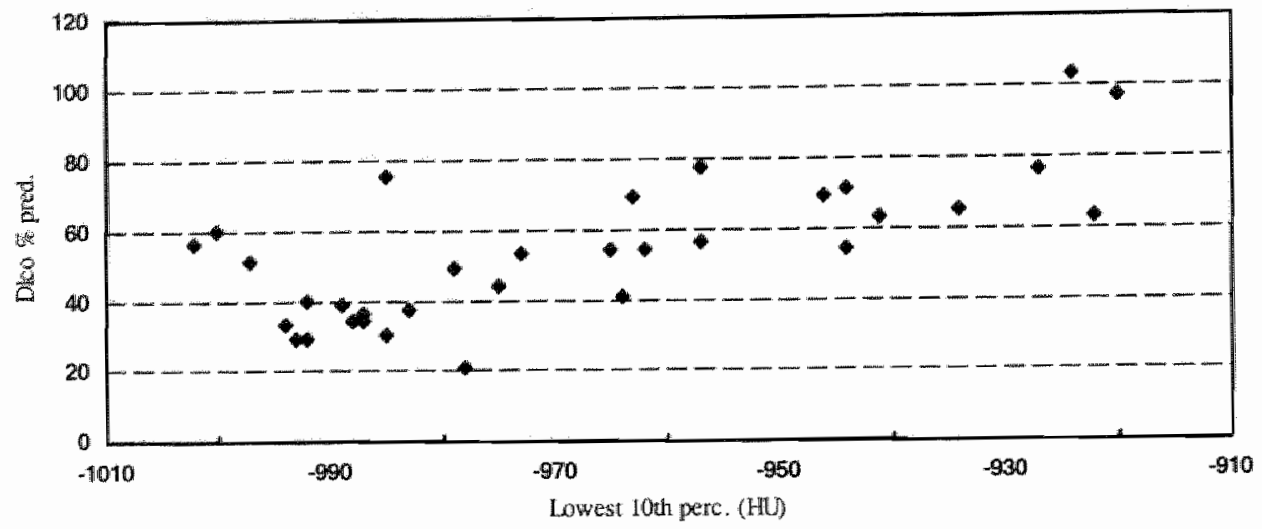

Figure 8. Relation between D1co \% predicted and the lowest 10 h precentile at $90 \%$ wital capacity in 33 patients with COPD and FV I less than IL.

$-t=0.70, p<0.0001$

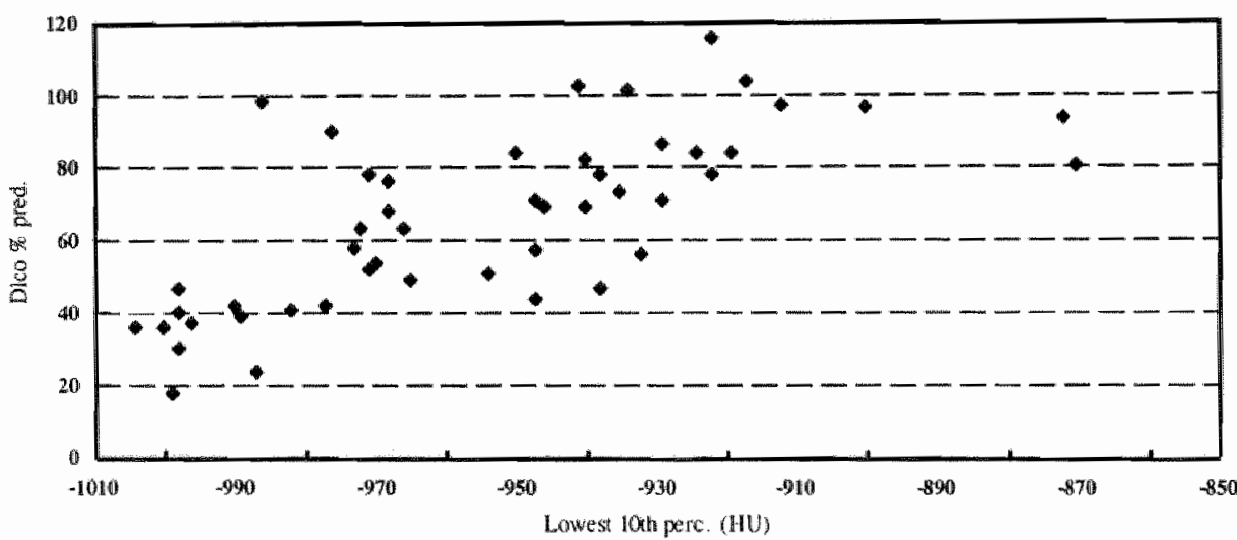

Figure 9. Relation between D 1 co 8 predicted and the lowest 10 th precentule al $90 \%$ vital capacity in 48 patients

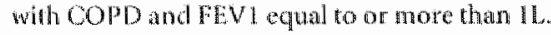

$\%$ predicted the CT emphysema score was $\geq 30$. In 43 of 53 patients $(81 \%)$ with DL $\mathrm{COO}_{\mathrm{CO}}$ $\geq 50 \%$ predicted the CT of the lung showed no abnormalities.

The relationship between $D L_{C O} \%$ predicted and the CT emphysema score in patients with FEV, levels $<1 \mathrm{~L}$ is shown in Figure $6(\mathrm{r}=-0.73, \mathrm{p}<0.0001)$. All of the 13 patients with a $\mathrm{FEV}_{1}<1 \mathrm{~L}$ and a $\mathrm{DL}_{\mathrm{CO}}<50 \%$ predicted had emphysematous parenchymal changes ( CT grade $\geq 30$ ). Seven of these 13 patients $(54 \%$ ) had significant emphysema (CT grade $\geq 60$ ). In 12 of 20 patients $(60 \%)$ with a $\mathrm{FEV}_{1}<\mathrm{IL}$ and a DL $\mathrm{CO}_{\mathrm{O}} \geq 50 \%$ pre- 
dicted, the CT of the lung was nomal. The relationship between DLCO $\%$ predicted and the CT emphysema score in patients with $F E V_{1}$ levels $\geq \mathrm{L}$ is illustrated in Figure 7 $(r=-0.62, p<0.0001)$. In 31 of 33 patients $(94 \%)$ with a $F E V_{1} \geq I L$ and a DL $C_{C O} \geq 50 \%$ $\%$ predicted, the CT of the lung showed no abnormalities. In 9 of 15 patients (60\%) with a FEV $1 \geq 1 \mathrm{~L}$ and a $\mathrm{DL}_{\mathrm{CO}}<50 \%$ predicted the CT scan of the lungs showed significant emphysematous changes. There was a significant correlation between $\mathrm{DL} \mathrm{CO}_{\mathrm{C}}$ predicted and the lowest 10 th percentile of the frequency distribution at $90 \%$ vital capacity in patients with a $\mathrm{FEV}_{1}<\mathrm{IL}(\mathrm{r}=0.72, \mathrm{p}<0.0001)$ (Figure 8), and also in patients with levels $F E V_{1} \geq I L(r=0.70, p<0.0001)$ (Figure 9).

The correlation between $\mathrm{DL} \mathbb{L}_{\mathrm{CO}} \%$ predicted and the CT emphysema score in patients with $\mathrm{FEV}_{1}$ levels $<50 \%$ predicted was -0.69 ( $\left.\mathrm{p}<0.001\right)$. In 24 of 25 patients (96\%) with a FEV $1<50 \%$ predicted and a $\mathrm{DL}_{\mathrm{CO}}<50 \%$ predicted, the CT emply sema score was $>30$. In 16 of these 25 patients $(64 \%)$ the CT emphysema score was $\geq$ 60 . In 23 of 32 patients $(72 \%)$ with a $\mathrm{FEV}_{1}<50 \%$ predicted and a $\mathrm{DL}_{\mathrm{CO}}>50 \%$ predicted, the CT of the lung was $<30$. The correlation between $\mathrm{DL}$ CO $\%$ predicted and the CT emphysema score in patients with a $F_{1} \geq 50 \%$ predicted was -0.32 (p< 0.05). In 19 of 22 patients $(86 \%)$ with a FEV $1 \geq 50 \%$ predicted and a DLCO $\geq 50 \%$ predicted, the CT emphysema score was $<30$. The CT emphysema score of all 4 patients with $\mathrm{FEV} \mathrm{V}_{1} \geq 50 \%$ predicted and a $\mathrm{DL}_{\mathrm{CO}}<50 \%$ predicted was $\geq 60$.

\section{DISCUSSION}

In the studied population of patients with COPD, airflow limitation and emphysema were fairly correlated. Forty-six percent of patients with stage III COPD (FEV $<35 \%$ predicted) had significant emphysema (CT grade $\geq 60$ ) as opposed to 4 percent of patients with stage I COPD $\left(50 \leq \mathrm{FEV}_{1}<70 \%\right.$ predicted). The same conclusion can be drawn from the correlation between $\mathrm{EEV}_{1}$ and $\mathrm{CT}$ densitometry of the lung. Airflow limitation and progression of emphysema was attended with lower attenuation values as measured on CT. Moreover, the carbon monoxide diffusing capacity (DLCO) was well related with the CT emphysema score and CT lung densitometry parameters in patients with COPD. In patients with advanced COPD $\left(F E V_{\mathbb{1}}<1 \mathrm{~L}\right)$, the CT emphysema score and lung densitometry correlated equally well with DL $\mathrm{CO} \%$ predicted. Resullts of the present study indicate a high prevalence of CT-scored significant emphysema in patients with COPD and DLCO \% predicted of less than 50 , and a low prevalence of CT-scored significant emphysema in patients with COPD and DLCO $\%$ predicted equal to or more than 50 . However, in patients with COPD and DL ${ }_{C O} \%$ predicted of less than 50 , the CT emphysema score and $\mathrm{CT}$ lung densitometry showed a significant but low correlation with $\mathrm{DL}_{\mathrm{CO}} \%$ predicted. The $\mathrm{DL}_{\mathrm{Co}}$ can not be relied on for quantitative assessment of severity and extent of emphysema. 
Using a visual grading method, CT-scored significant emphysema was found mainly in patients with COPD with stage II or stage III COPD (FEV $1<50 \%$ predicted). In contrast, in $85 \%$ of patients with COPD with stage I COPD, the CT of the chest showed no abnormalities. The CT emphysema score in patients with advanced COPD was significantly higher than the CT emphysema score in patients with COPD and FEV equal or more than $1 \mathrm{~L}$. Our data confirm the conclusions reached by Miniati et al who found a high prevalence of emphysema as indicated by CT scores in patients with adwanced COPD (33) but differ substantially from previous published data from Gelb et al who reported a limited presence of and role for emphysema in causing chronic expiratory airflow limitation in patients with advanced chronic obstructive lung disease (4). We have no unequivocal explanation for the difference in results between both studies. Both study populations showed a great resemblance with respect to age, indicators of airflow limitation, as well as $\mathrm{DLCO}_{\mathrm{CO}} \%$ predicted values. However, in our heterogeneous population of 100 patients with COPD the full range of $\mathrm{CT}$ emphysema scores was well-represented whereas in their study population, patients with severe emphysema as indicated by CT scores were probably under-represented.

In an effort to provide skill independent support to our visual observations we used CT densitometry to objectively quantify the extent of pulmonary emphysema. This is feasible since the performance of modern CT scanners is quite adequate for densitometry of the lungs (34). The stability of the CT system at air densities is within $1 \mathrm{HU}$, and reproducibility and accuracy for densities found for lung tissue were within 1-2 HU (35). Moreover, quantitative analysis of expiratory CT images has proved useful in assessing degrees of air-trapping in diseases affecting the airways. CT scans obtained during suspended full expiration in patients with small airways disease show focal air retention in lung parenchyma which can be densitometrically quantified $(31,32,36)$. Air-trapping during exhalation in patients with COPD is reflected by a reduction in the change between the densitometric data obtained at inspiration and at expiration (37).

Lung density measurements obtained in the present study confirmed visual observations that patients with significant emphysematous destruction of lung parenchyma are found mainly in the patients with stage II and stage III COPD. Furthermore, with progression of airflow limitation, the lung density in patients with COPD obtained at $90 \%$ vital capacity and at $10 \%$ vital capacity decreased as expected. The change between the densitometric data obtained at both levels of inspiration in the group of patients with stage III COPD was significantly smaller than in patients with stage I COPD. Similar observations were obtained after stratification of the COPD population based on the degree of CT-scored emphysema. With increasing CT-scored emphysema, the mean lung density at $90 \%$ and $10 \%$ vital capacity CT numbers decreased progressively as expected. Besides, the change between the densitometric data obtained at $90 \%$ vital capacity and at $10 \%$ vital capacity was in the group of patients with 
COPD and severe emphysema (CT grade $\geq 90$ ) significantly smaller than in patients with COPD with no or trivial emphysema (CT grade $<30$ ). This smaller change between the densitometric data observed in patients with severe emphysema provides additional support to our hypothesis that emphysema is related to airflow limitation.

Quantitative techniques must be carefully controlled. Submaximal efforts have major effect on attenuation measurements as lung density values vary inversely with lung volume. We applied a spirometric gated CT technique which offers the opportunity to scan at defined levels of inspiration (25). A spirometric curve can be followed on the display monitor of the CT scanner. Feedback control makes it easier to coach patients for a truly maximal effort. CT densitometry of the lung is usually performed at full inspiration or at full expiration. However, maximal effort is particularly fatiguing for patients with advanced airflow limitation. Therefore, we scanned, for the convenience of our patients at $90 \%$ and $10 \%$ vital capacity trigger level.

The $\mathrm{DL}_{\mathrm{CO}}$, whether measured by single-breath or steady state method, expressed in absolute terms, as per cent predicted, or as function of alveolar volume, has constantly been considered as the best predictor of severity of emphysema. However, wide variations in correlation between $\mathrm{DL}_{\mathrm{CO}}$ and the macroscopic extent of emphysema have been reported $(27,38)$. We found a good correlation $(r=-0.72, p<0.0001)$ between $\mathrm{DL}_{\mathrm{CO}}$ and the $\mathrm{CT}$ emphysema score in our population of patients with COPD. These results are in agreement with Gould et al (38) who found a similar inverse correlation between $\mathrm{DL}_{\mathrm{CO}}$ and the lowest 10 th percentile in a group of 80 subjects that ranged from normal subjects to patients with chronic respiratory failure. In another study, these authors reported a similar inverse correlation between the lowest 10th percentile of non-bullous lung and $\mathrm{DL}_{\mathrm{CO}}$ in a group of 23 patients with varying degrees of emphysema and in addition bullous disease (39).

Gelb and coworkers question the usefulness of the $\mathrm{DL}_{\mathrm{CO}}$ as indicator of significant emphysema (4). They found poor correlation between $\mathrm{DL}_{\mathrm{CO}}$ and the CT emphysema grade in 23 patients with advanced airflow limitation defined as FEV, levels less than 1L. Opposite to these results, we found a good correlation between DL $\mathrm{L}_{\mathrm{CO}}$ and the severity of emphysema independent of the degree of airflow limitation. Correlations were even higher in patients with advanced airflow limitation. Nevertheless, in our opinion, the $\mathrm{DL}_{\mathrm{CO}}$ is a useful test to separate patients with COPD with emphysema from patients with COPD without emphysema. The vast majority of patients $(93 \%)$ with COPD and a DLCO \% predicted of less than $50 \%$ showed emphysematous changes at CT, and $56 \%$ of these patients suffered from significant emphysema. Deviating from this, $23 \%$ of patients with $\mathrm{COPD}$ and $\mathrm{DL}_{\mathrm{CO}} \%$ predicted of equal to or more than 50 showed emphysema on CT, and only a small minority of patients ( $4 \%$ ) showed significant emphysematous parenchymal changes on CT.

In conclusion: Results suggest a distinct presence and degree of emphysema in patients with advanced COPD. The DL $\mathrm{CO}$ is a reliable indicator of emphysema also in 
patients with advanced chronic obstructive pulmonary disease. A high prevalence of CT-scored emphysema was observed in patients with COPD and DL $\mathrm{CO} \%$ predicted of less than 50 . However, $\mathrm{DL}_{\mathrm{CO}}$ measurements cannot be relied on for quantitative assessment of severity and extent of emphysema. $\mathrm{DL}_{\mathrm{CO}}$ measurements are therefore useful measurements to select patients for radiologic emphysema assessment. Further prospective studies are needed to validate this stratification criterium.

\section{REFERENCES}

1. ATS Statement. Standards for the diagnosis and care of patients with chronic obstructive pulmonary disease. Am J Respir Crit Care Med 1995; 152:\$77 \$120.

2. Mead JJM, IIumer JM, Macklem PT, Little IB. Significance of the relationship between lung recoil and maximum expiratory flow. J Appl Physiol 1967;22:95-108.

3. Kim WD, Eidelman DH, Izquierdo JL, Ghezzo H, Saetta MP, Cosio MG. Centrilobular and panlobular emphysena in snokers: two distinct morphologic and functional entities. Am Rev Respir Dis 1991; 144:1385-1390.

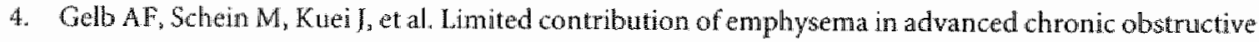
pulmonary disease. Am Rev Respir Dis 1993; 147:1157-1161.

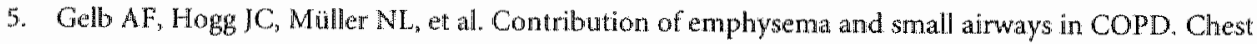
$1996 ; 109: 353-359$.

6. Hogg JC. Macklem PT, Thurlbeck WM. Site and nature of airway obstruction in chronic obstructive lung disease. N Engl J Med 1968; 278:1355-1360.

7. Cosio MG, Ghezzo H, Hogg JC, Corbin R, Loveland M, Dosman JJ, Macklem PT. The relations between structural thanges in small airways and pulmonary function tests. N Engl I Med 1978; 298:1277\%1281.

8. Wright J, Lawson LM, Paré PD, Kennedty S, Wiggs B, Hogg JC. The detection of small airways disease. Am Rev Respir Dis 1984; 129:989-994.

9. Berend $\mathrm{N}$, Wright JL, "Thurlbeck WM, Marlin GE, Woolcock AJ. Small airways disease: reproducibility of measurements and correlation with lung function. Chest 1981; 79:263-268.

10. Hogg IC, IL Wright JL. Wiggs BR, Coxson HO, Opazo Saez A, Paré PD. Lung structure and function in cigarette smokers. Thorax 1994; 49:473-478.

11. Thurbeck WM, Henderson JA, Fraser RG. Bates DV. Chronic obstructive lung disease: a comparison between clinical, roentgenologic, functional, and morphologic criteria in chronic bronchitis, emphy sema, asthma, and bronchiectusis. Medicine 1970; 49:81-145.

12. Symonds $G$, Renzetti AD, Mitchell MM. The diffusing capacity in pulmonary emphysema. Am Rev Respir Dis 1974; 109:391-394.

13. West WW. Nagai A, Hodgkin JE, Thurbeck WM. The National Institutes of Healuh intermittent positive pressure breathing trial. Pathological studies. Am Rev Respir Dis 1987; 135:123-129.

14. Pratt $\mathrm{PC}$. Kilburn. $\mathrm{KH}$. A modern concept of the emphysemas based on correlations of structure and function. Hum Pathol $1970 ; 1: 433-463$.

15. Stern EJ, Frank MS. CE of the lung in patients with pulmonary emphysema: diagnosis, quantification, and correlation with pathologic and physiologic findings. A.JR 1994; 162:791-798.

16. Thurlbeck WM, Müller NL. Emphyseraa: definition, imaging, and quantification. AJR 1994; $163: 1017-1025$.

17. Gevenois PA, Yernault JC. Can computed tomography quantify pulmonary emphysema. Eur Respir J $1995 ; 5 ; 843-848$. 
18. Morgan MDL. Detection and quantification of pulmonary emphysema by computed tomography: a window of opportunity. Thorax 1992; 47:1001-1004.

19. Lamers RJS, Kemerink GJ, Drent M, van Engelshoven IMA. Reproducibility of spirometrically controlled CT lung densitometry in a clinical setting. Eur Respir I 1988; 11:942-945.

20. American Thoracic Society. Chronic bronchitis, asthma, and pulmonary emphysema: a statement by the Committee on Diagnostic Standards for Nontuberculous Respiratory Disenses. Am Rev Respir Dis 1962; 85:762-768.

21. Quanjer PH. Standardized lung function testing. Bull Eur Physiopathol Respir 1983; 19:7-44.

22. Quanjer P, Tammeling GI, Cotes IE, Pedersen OF, Peslin R, Yernault JC. Lung volumes and forced ventilatory flows. Official statement of the European Respiratory Society. Eur Respir I 1993; 16 (Suppl 6):5-40.

23. Sakai F, Gamsu G. Im JG, Ray CS. Pulmonary function abnormalities in patients with Cl-determined emphysema. J Comput Assist Tomogr 1987; 11:963-968.

24. Kemerink GI, Kruize HH, Lamers RJS. The CT's sample volume as an approximate, instrumental measure for density resolution in densitometry of the lung. Med Phys 1997; 24:1615-1620.

25. Kalender WA, Rienmuller R, Seissler W, Behr J, Welke M, Fichte H. Measurement of pulmonary parenchymal attenuation: use of spirometric gating with quantitative CT. Radiology 1990; 175:265-268.

26. Pavlidis T. Algorithms, for graphics and image processing. Springer Verlag* Berlin. 1982.

27. Gould GA, Macnee W, McLean A, et al. CT measurements of lung density in life can quantitate distal airspace enlargement: an essential defining feature of human emphysena. Am. Rev Respir Dis 1988; 137:380-392.

28. Biernacki W, Gould GA, Whyte KF, Fleniey DC. Pulmonary hemodynamics, gas exchange, and the severity of emphysema as assessed by quantitative CT' scan in chronic bronchitis and emphysema. Am Rev Respir Dis 1989; 139:1509-1515.

29. Heremans A, Verschakelen JA, Van Fraeyenhoven L, Demedts M. Measurement of lung density by means of quantitative CT scanning. Chest 1992; 102:805-811.

30. Beinert $T$, Behr F, Mehnert $F_{n}$ et al Spirometrically controlled quantitative CI' for assessing diffuse parenchymal lung disease. J Comput Assist Tomogr 1995:19:924-931.

31. Newman KB, Lynch DA, Newman LS, Ellegood D, Newell ID. Quantiative computed tomography dettects air trapping due to asthma. Chest 1994; 1.06:105-109.

32. Gevenois PA, De Vuyst $P$, Sy M, et al. Pulmonary emphysema: quantitative CT during expiration. Radiology $1996 ; 199: 825-829$.

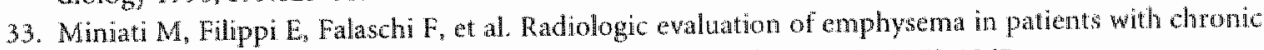
obstructive pulmonary disease. Am I Respir Crit Care Med 1985: 151:1359-1367.

34. Kemerink G], Lamers RIS, Thelissen GRP, wan Engelshoven IMA. Scanner conformity in CI densi tometry of the lungs. Radiology 1995; 197:749-752.

35. Kemerink GI, Lamers RJS, Thelissen GRP, van Engelshoven IMA. CT densitometry of the lungs: scanner performance. I Comput Assist Tomogr 1996: 20:24-33.

36. Eda S, Kubo K, Fujimoto K, Matsuzawa X, Sekiguchi M, Sakai F. "The relations between expiratory chest CT using helical CT and pulmonary function tests in emphysema. Am J Respir Crit Care Med $1997 ; 155: 1290-1294$.

37. Lamers RIS, Thelissen GRP, Kessels AG, Wouters EM, van Engelshoven JMA. Chronic obstuctive pulmonary disease: evaluation with spirometrically controlled CT lung densitometry. Radiology 1994; 1993:109-113.

38. Gould $G A$, Redpath $A T$, Ryan $M$, et all. Lung CT density correlates with meastrements of airflow limitation and the diffusing capacity. Eur Respir $\| 1991 ; 4: 141-146$.

39. Gould GA, Redpath AT, Ryan M, et al. Parenchymal emphysema measured by CT lung density correlates with lung function in patients with bullous disease. Eur Respir $\$ 1993 ; 6: 698-704$. 
CHAPTER 9

\section{CT lung densitometry and visual assessment of thin-section CT in the diagnosis of coal worker's pneumoconiosis}

Rob J.S. Lamers

Paul ).A. Borm

Alfons G.H. Kessells

Gerrit). Kemerink

Emiel F.M. Wouters

Jos M.A. van Engelshoven 


\section{ABSTRACT}

Purpose: To assess the usefulness of computed tomographic (CT) hung densitometry in coal worker's pheumoconiosis (CWP).

Materials and methods: Spirometrically gated CT sections were obtained at $5 \mathrm{~cm}$ above and $5 \mathrm{~cm}$ below the carina at $90 \%$ and $10 \%$ vital capacity $(\mathrm{VC}$ ) in a group of 66 persons consisting of 35 coal miners with a normal chest radiograph, 11 coal miners with a chest radiograph showing nodular opacities consistent with CWP, and 20 healthy wolunteers. Various densitometric parameters were derived from the CT data. Additionally, thin-section CT scans were obtained in the upper and the lower zones of the lungs, and wisually assessed for the presence and extent of CWP. Based on results of visual assessment, subjects were divided into three subsets. Densitometric parameters of CWP between the three groups were compared and correlated with results of visual assessment of $\mathrm{CT}$ scans at the same anatomical levels.

Results: With increasing extent of CWP higher densitometric values were observed at both levels of inspiration. Densitometric parameters between groups differed significantly in the upper zones only at $90 \%$ VC (all $p>0.01$ ). Good correlations between objective and subjective parameters of CWP were observed at $90 \% \mathrm{VC}$, being best in the upper zones. At 10\% VC, correlations were always poor.

Conclusion: CT scans obtained at $90 \% \mathrm{VC}$ in the upper zones of the lungs are the best candidates for lung densitometric measurements in CWP. Objective measurements of lung density correlate well with direct observational assessment of CWP provided that CT sections are obtained at $90 \% \mathrm{VC}$.

\section{INTRODUCTION}

Coal worker's pneumoconiosis is usually diagnosed from a history of significant coal dust exposure and a combination of clinical, functional and radiographic findings. The chest radiograph appearance, coded according to the Intermational Labor Organization (LLO 1980) criteria, is generally accepted as evidence of the presence of coal worker's pneumoconiosis without histological proof ( 1 ). However, the ILO classified chest radiograph may underestimate the presence of interstitial lung disease (2). Chest radiographs have a low sensitivity for detecting minimal to moderate grades of coal worker's pneumoconiosis (3). Visual assessment of thick-section computed tomography (CT) images has proven superior to the chest radiograph in the early detection of diffuse parenchymal lung diseases $(4 * 6)$. Thin-section CT identifies even significantly more parenchymal opacities than thick-section CT (7-9). However, visual assessment of nodules at thin-section $\mathrm{CT}$ scans of the lungs was shown to be subject to interobserver variability $(9)$. 
Lung density measurements have the advantage of providing quantitative data automatically, thereby eliminating observer variability. Performance of modern generation CT scanners compare favorable with that reported in the past (10-12). Scanner conformity, reproducibility and accuracy of present CT scanners is adequate $(13,14)$. The value of this technique is clearly proven in pulmonary emphysema where densitometric assessment correlates well with the pathologic score of emphysema (15-17). Assessment of parenchymal density on CT is a valid measure of the extent of interstitial lung disease $(18,19)$. The physical density of the lung parenchyma increases proportionally to the amount of silicotic nodules present. As density changes rapidly and markedly with the level of inspiration, the necessity of reproducible levels of inspiration was a major limitation. This problem has been solved by applying spirometric control of the level of inspiration for gating the CT scanner (20).

Radiographic documentation of coal worker's pneumoconiosis is important for diagnosis, surveillance, compensation, and disease prevention (3). During a five year follow-up period in a group of 104 retired coal miners, a significant portion of this cohort showed progression of coal worker's pneumoconiosis on chest radiographs (21). Quantitative analysis of the frequency distribution of Hounsfield numbers defines the radiological extent and/or progression of coal worker's pneumoconiosis related to parenchymal changes by comparing results from base line to follow-up scans. Patients with other interstitial lung diseases, with diffuse fibrosing alveolitis and granulonatous lung disease, showing normal lung function tests, have been proven to differ significantly from healthy controls in the frequency distribution of CT numbers (22).

This study assesses coal worker's pneumoconiosis by (a) comparing the results of spirometrically controlled CT lung densitometry scans obtained from three groups of subjects: coal miners with a mild abnormal thin-section CT suggestive of coal worker's pneumoconiosis, coal miners with a more severe abnormal thin-section $\mathrm{CT}$ consistent with coal worker's pneumoconiosis, and persons with a normal thin-section CT and (b) relating results of spirometrically gated CT lung densitometry with those of visual assessment of thin-section CT scans. For this purpose, measurements in the upper and the lower zones of the lungs at two defined levels of inspiration were performed.

\section{MATERIALS AND METHODS}

The initial study population consisted of 156 retired coal worker's who worked underground in the Belgian collieries for more than 20 years and who were involved in previous studies $(23,24)$. Standard high-kilovoltage posteroanterior and lateral chest radiographs were obtained at maximal inspiration. Chest radiographs were read by a panel of three board medical officers, with a long term familiarity in reading and clas- 
sifying pneumoconiosis according to ILO criteria for compensation purposes (1). Differences in opinion were resolwed by consensus.

Based on these chest radiograph results, 35 coal miners aged $40-61$ years (mean \pm standard deviation, $47 \pm 8$ years) with a normal chest radiograph (ILO $\leq 0 / 1$ ), and 11 coal miners aged $42-56$ years ( $51 \pm 5$ years) with small rounded opacities (ILO $>0 / 1$ ) consistent with simple coal worker's pneumoconiosis, were randomly selected and invited to participate. A third group consisted of 20 non-exposed volunteers aged 31 - 78 years ( $53 \pm 12$ years), who had neither pulmonary symptoms nor any history of pulmonary diseases. Pulmonary function tests i.e. forced expiratory volume in I second $\left(\mathbb{F E V}_{1}\right)$ and transfer coefficient $\left(\mathrm{K}_{\mathrm{CO}}\right)$ were both more than $80 \%$ of the predicted value in the latter group. Subject characteristics of our study groups (Table 1) showed that coal miners with a normal chest radiograph, coal miners with coal worker's pneumoconiosis and healthy volunteers were not different in age, length, weight and smoking history. Written informed consent was obtained from all participants. The study was approved by the local medical ethics committee.

Occupational history data were obtained from questionnaires and confirmed by personal interviews. The number of years underground in coal miners with a normal chest radiograph was significantly lower $(\mathrm{p}<0.005)$ compared to the number of years underground of coal miners with coal worker's pneumoconiosis. The cumulative dust exposure was calculated as the sum of the products of the yearly mean dust concentration for the colliery where the miner had worked and the average time worked underground during that year. The resulting individual units were expressed as gram-hours per cubic meter of sampled air (21).

\section{Pulmonary function studies}

Pulmonary function tests were performed at the day of $\mathrm{CT}$ densitometry. Spirometric measurements were obtained by means of a wet spirometer (Gould Pulmonet III: Sensormedics, Bilthoven, the Netherlands): $F E V_{1}$ was expressed as a percentage of the reference values (25). Lung volumes were measured by whole-body plethysmography and were again expressed as a percentage of the reference values (25). The diffusing capacity was measured by the single-breath method (MasterLab; Jaeger, Würzburg, Germany). $K_{C o}$ was calculated as diffusing capacity divided by the alveolar volume.

\section{Visual assessment of CT scans}

In each person, four thin-section CT scans were obtained (Somatom Plus; Siemens, Erlangen, Germany) within two year of the chest radiograph, at end-inspiratory lung volume with the person in supine position; two scans of the upper zones, 3 and $5 \mathrm{~cm}$ above the carina, and two scans of the lower zones, 3 and $5 \mathrm{~cm}$ below the carina. Scan- 
ning parameters were $1.0-\mathrm{mm}$ collimation, $137 \mathrm{kVp}, 220 \mathrm{~mA}, 1.0$ second scanning time, high-resolution reconstruction algorithm. Images were photographed at window level and width appropriate for lung detail $(-800,1600 \mathrm{HU})$. Each slice was scored by two thoracic radiologists (R.J.S.L., J.M.A.v.E.), separately, for the presence of noncalcified parenchymal opacities and categorized using the same principles as the ILO system for pulmonary disease on chest radiographs (1). This approach was used previously (8). Four profusion scores were defined: 0 , no abnormalities; 1 , few nodules, normal lung markings visible; 2 , moderate opacities, normal lung markings partially obscured; 3, numerous opacities, normal lung markings totally obscured. The profusion score was noted as follows and converted to a linear scale from 0 to 10:0/0 $=0,0 / 1$ $=1,1 / 0=2,1 / 1=3,1 / 2=4,2 / 1=5,2 / 2=6,2 / 3=7,3 / 2=8,3 / 3=9,3 / 4=10$. In the profusion score the first digit represents the final score and the digit behind the slash the alternative score the observer doubted. This classification represents a continuum of change from no opacity to the most advanced category. The score of the eight lung areas - four CT slices multiplied by two lungs - was summed and a total score was calculated, which could range from zero to 80 . The coal worker's pneumoconiosis score for the whole lung as well as for the upper and the lower zones of the lungs were determined by each of the two observers and these scores were averaged. Both radiologists were blinded to the chest radiograph results and the exposure history when interpreting thin-section CT's. CT scans were also scored for the presence or absence of progressive massive fibrosis or post-tuberculous scars as well as for the presence of emphysematous changes. The CT diagnosis of emphysema was based on the presence of areas of abnormally low attenuation as determined visually (26).

\section{Quantitative assessment of CT scans}

The CT scans of the lungs for quantitative assessment of lung density patterns were obtained in supine position. Scanning parameters were $1.0-\mathrm{mm}$ collimation, $137 \mathrm{kVp}$, $220 \mathrm{~mA}$ and 1.0 -second scanning time. Scans were reconstructed in the standardresolution mode. No contrast medium was injected. In order to control the level of inspiration during scanning, the patient was asked to breathe through a small handheld spirometer (Micro Medical Instruments, Rochester, England), which was connected with the CT scanner (20). At user-selected levels of inspiration air flow was interrupted mechanically by a closing valve at what time a trigger signal was generated to start the scanner. The level of inspiration was constant for the duration the CT scan was made (20). Two anatomical levels were selected from the topogram: $5 \mathrm{~cm}$ above and $5 \mathrm{~cm}$ below the level of the carina. At each level two scans at $90 \%$ and $10 \%$ inspiratory vital capacity, respectively, were obtained. The CT data were digitally transferred for analysis to a workstation (Sun SPARCstation 1+; SUN Microsystems, Mountain View, Calif). After the parenchyma of both lungs was delineated automatically by a 
density-discriminating computer program, three densitometric parameters were derived: the mean lung density, the cut-off point of the frequency distribution (Hounsfield unit number) that defines the lowest 10th percentile, and the cut-off point that defines the highest 10 th percentile. Apart from nodules, the physical density of the lung in coal worker's pneumoconiosis is determined by three other components: lung tissue, blood vessels, and air. Silicotic nodules, blood vessels and airway walls are densitometrically reflected by the Hounsfield number defining the highest 10 th percentile of the frequency distribution of Hounsfield numbers whereas the Hounsfield number defining the lowest 10th percentile is more likely to represent air containing tissue.

\section{Statistical analysis}

Results of visual assessment of both radiologists were compared with the Spearman rank correlation coefficient. The Student $t$ test with a two-tailed significance level of 0.05 was used to evaluate the intergroup differences in parameters from spirometrically controlled CT lung densitometry and pulmonary function tests. Correlations between the coal worker's pneumoconiosis score for the upper and the lower zones and quantitative lung density parameters as well as the correlation between the coal worker's pneumoconiosis score of the whole lung and cumulative dust exposure were described by the Pearson correlation coefficient. For their correlation coefficients one-tailed $\mathrm{p}$ values were calculated.

\section{RESULTS}

Cumulative dust exposure of coal miners with a normal chest radiograph was $84 \pm 51$ $\mathrm{gh} / \mathrm{m}^{3}$ and tended to be lower in comparison to coal miners with coal worker's pneumoconiosis $\left(122 \pm 58 \mathrm{gh} / \mathrm{m}^{3}\right)$. No significant differences in pulmonary function tests were observed between coal miners with a normal chest radiograph, coal miners with a chest radiograph showing nodular opacities consistent with coal worker's pneumoconiosis and healthy volunteers (Table 1).

Visual assessment of thin-section CT scans showed a good interreader correlation for the presence of nodular infiltration $(r=0.80, p<0.0001)$. The median coal worker's pneumoconiosis score of the whole lung of coal miners with a normal chest radiograph was 7 (range, 0-39) and of coal miners with a chest radiograph showing nodules consistent with coal worker's pneumoconiosis 22 (range, 2-51). The median coal worker's pneumoconiosis score for the upper zones of the lungs in the whole coal miners population ( $\mathrm{n}=46$ ) was 7 (range, 0-35,5) and for the lower zones of the lungs 5 (range, 0-20.5). Since CT images allow a better stratification of parenchymal opacities 
Table I. Subjects Characteristics.

\begin{tabular}{|c|c|c|c|c|c|c|c|}
\hline \multirow[b]{2}{*}{ Parameter } & \multicolumn{3}{|c|}{$\begin{array}{l}\text { Coal miners without CWP } \\
(1 L O \leq 0 / 1) \\
(\mathrm{n}=35)\end{array}$} & \multicolumn{2}{|c|}{$\begin{array}{l}\text { Coal miners with CWP: } \\
(\mathrm{LO}>0 / 1) \\
(n=11)\end{array}$} & \multicolumn{2}{|c|}{$\begin{array}{l}\text { Healthy volunteers } \\
(\mathrm{n}=20)\end{array}$} \\
\hline & Mean & SD & $p^{*}$ & Mean & $\mathrm{SD}$ & Mean & $\mathrm{SD}$ \\
\hline $\operatorname{Age}(Y)$ & 47 & 8 & & 51 & 5 & 53 & 12 \\
\hline Length $(\mathrm{cm})$ & 170 & 8 & & 173 & 6 & 175 & 10 \\
\hline Weight (kg) & 77 & 11 & & 84 & 11 & 78 & 10 \\
\hline Years underground & 22 & 3 & $<0.005$ & 26 & 3 & 0 & \\
\hline Cig. cons. (pack years) & 23 & 23 & & 31 & 21 & 17 & 12 \\
\hline FEV, (\% predicted) & 106 & 16 & & 100 & 15 & 110 & 18 \\
\hline TLC (\% predicted) & 101 & 13 & & 97 & 11 & 100 & 11 \\
\hline DL $(\%$ predicted) & 117 & 22 & & 109 & 38 & 111 & 19 \\
\hline $\mathrm{K}_{\mathrm{cos}}(\%$ predicted) & 88 & 15 & & 84 & 27 & 90 & 9 \\
\hline
\end{tabular}

Note. CWP = coal worker's pneumoconiosis, $1 \mathrm{LO}=$ International Labor Organisation, values represent mean \pm standard deviation; ${ }^{*}$ Coal miners $L \mathrm{LO} \leq 0 / 1$ versus coal miners $\mathrm{L} \mathrm{LO}>0 / 1$.

than chest radiographs, a comparison of densitometric and visual assessment of pneumoconiosis should preferably rely on visual scores from the CT modality. Our study population $(\mathrm{n}=66$ ) was therefore arbitrarily subdivided into three groups; group 1, normal thin-section CT scan (coal worker's pneumoconiosis score $=0$ ), $n=27$; group 2 , mild abnormal thin-section CT suggestive of coal worker's pneumoconiosis (coal worker"s pneumoconiosis score $0<\mathrm{X} \leq 15$ ), $\mathrm{n}=22$; and group 3, more severe abnormal thin-section. CT consistent with coal worker's pneumoconiosis (coal worker's pneumoconiosis score $>15$; range, 16-52) $\mathrm{n}=17$. The median of the coal worker's pneumoconiosis score for the upper zones of the lungs of group 2 was 3.5 (range, $0-10.5$ ) and 12 (range, 2-35.5) in group 3. The median of the coal worker's pneumoconiosis score for the lower zones of the lungs of both groups was 1 (range, 0-6,5) and 12 (range, 4-20.5) respectively. Coalescence of opacities or large opacities were not observed. Two coal miners, one with a coal worker's pneumoconiosis score of 0 , and one with coal worker's pneumoconiosis of 14 , showed mild changes of centriacinar emphysema at thin-section CT.

Pulmonary function tests in the 3 groups were within normal limits (Table 2). Lung function tests gradually decreased with increasing coal worker's pneumoconiosis score of the whole lung, though not significant except for $\mathrm{K}_{\mathrm{Co}}$ between group $\mathrm{I}$ and group $3(\mathrm{p}<0.05)$.

The results of the densitometric readings in the three groups are summarized in Table 3. All three densitometric parameters increase with increasing coal worker's pneumoconiosis score. At $90 \%$ vital capacity, the mean lung density, the lowest 10 th percentile 
Table 2. Rewultw of Pumonary Function Tests for Study Population Subdivided According to the Coal Worker's Preumoconiosos Score of the thole Lung.

\begin{tabular}{|c|c|c|c|c|c|c|c|}
\hline \multirow{2}{*}{ CWp score } & \multicolumn{2}{|c|}{$\begin{array}{l}\text { Group } 1 \\
(n=27) \\
0\end{array}$} & \multicolumn{2}{|c|}{$\begin{array}{l}\text { Group } 2 \\
(n=22) \\
0<x \leq 15\end{array}$} & \multicolumn{3}{|c|}{$\begin{array}{l}\text { Group } 3 \\
(m=17) \\
>15\end{array}$} \\
\hline & Mean & $\mathrm{SD}$ & Mean & $S D$ & Mean & $\mathrm{SD}$ & $p^{*}$ \\
\hline FEV I $\%$ predicted) & 110 & 15 & 104 & 16 & 103 & 18 & \\
\hline TLC (o predicted) & 104 & 13 & 200 & 11 & 98 & 13 & \\
\hline$D I_{w_{h},}(\%$ predicted $)$ & 114 & 17 & 111 & 30 & 103 & 24 & \\
\hline Kco (\% predicted) & 90 & 9 & 92 & 22 & 81 & 16 & $<0.05$ \\
\hline
\end{tabular}

Note. CWD soore = coal worker's pneumoconiosis score, values represent mean \pm standard deviation;

* group 3 wersus group 1.

Table 3. Densitometric Results in the Study Population Subdivided According to the Coal Worker's Pnet. moconiosis Scora of the Whole lung at $90 \%$ and $10 \%$ Wital Capacity.

\begin{tabular}{|c|c|c|c|c|c|c|c|c|c|}
\hline \multirow{2}{*}{$\begin{array}{l}\text { CWP score } \\
\text { Vital Capacity }\end{array}$} & \multicolumn{3}{|c|}{$\begin{array}{l}\text { Group I } \\
(n=27) \\
0\end{array}$} & \multicolumn{3}{|c|}{$\begin{array}{l}\text { Group } 2 \\
(n=22) \\
0<x \leq 15\end{array}$} & \multicolumn{3}{|c|}{$\begin{array}{l}\text { Group } 3 \\
(n=17) \\
>1.5\end{array}$} \\
\hline & Mean & $\mathrm{SD}$ & $p^{*}$ & Mean & $\mathrm{SD}$ & $\mathrm{p}^{1}$ & Mean & $\mathrm{SD}$ & $\mathrm{p}$ \\
\hline \multicolumn{10}{|c|}{ Upper lang zomes } \\
\hline \multicolumn{10}{|c|}{ Mean lung density } \\
\hline-90 & -856 & 26 & & -842 & 17 & $<0.005$ & -803 & 48 & $<.0005$ \\
\hline-10 & -788 & 49 & & -772 & 58 & & -751 & 72 & \\
\hline \multicolumn{10}{|c|}{ Highest 10 th percentile } \\
\hline-90 & -751 & 51 & & -731 & 31 & $<0.005$ & -671 & 49 & $<0.001$ \\
\hline-10 & -642 & 74 & & -627 & 84 & & -593 & 93 & \\
\hline \multicolumn{10}{|c|}{ lowest loth percentile } \\
\hline-90 & -939 & 35 & & .927 & 12 & $<0.01$ & -908 & 27 & $<0.05$ \\
\hline-10 & -896 & 42 & & -883 & 50 & & -869 & 47 & \\
\hline \multicolumn{10}{|c|}{ Lower lung zones HU } \\
\hline \multicolumn{10}{|c|}{ Mean Jung density } \\
\hline-90 & -844 & 30 & $<0.005$ & -817 & 23 & & -804 & 30 & $<0.001$ \\
\hline-10 & -776 & 57 & & -768 & 59 & & -742 & 85 & \\
\hline \multicolumn{10}{|c|}{ Highest loth parcentile } \\
\hline-90 & -693 & 58 & & -666 & 42 & & -641 & 58 & $<0.01$ \\
\hline-10 & -569 & 101 & & .564 & 95 & & -556 & 85 & \\
\hline \multicolumn{10}{|c|}{ Lowest 10 h percentile } \\
\hline-90 & -948 & 20 & $<0,001$ & -925 & 17 & & -920 & 18 & $<0.001$ \\
\hline-10 & -910 & 38 & & .898 & 30 & & -891 & 38 & \\
\hline
\end{tabular}

Note. CWP score = coal worker's pneumoconiosis score, values represent mean intandard deviation and arexpressed in Hounstield units; $p^{*}$ Group 1 versus group 2; p Group 2 versus group $3 ;$ pt Group 3 versus group 1. 
of the frequency distribution, and the highest 10 th pecentile were in group 1 sigmificantly lower than in group 3 in both zones of the lungs. At $10 \%$ vital capacity no statistically significant differences between the two groups were observed. All three densitometric parameters were statistically different between the groups 2 and 3 if the measurements were performed at $90 \%$ vital capacity in the upper zones of the lungs (all $p<0.05$ ). In the lower zones of the lungs the mean lung density as well as the lowest 10 th percentile were significantly lower in group 1 compared with the two other groups, once again only at $90 \%$ vital capacity.

The best correlations between visual assessment of thin-section CT scans and CT lung densitometry at the same anatomical level were found in the upper zones of the lungs at $90 \%$ vital capacity (Table 4 ). In the lower lung zones correlations were moderate but significant. the mean lung density was the densitometric parameter that correlated best with the coal worker's pneumoconiosis score $(r=0.76, p<0.001 ; r=0.53, p<$ 0.001 respectively). At $10 \%$ vital capacity correlations between results of visual assessment of thin-section CT scans and CT lung densitometry were poor. Within individual groups, the best correlations between densitometry and visual assessment were observed in coal miners with a more severe abnormal thin-section CT consistent with coal worker's pneumoconiosis at $90 \%$ vital capacity (Table 5). There was no significant correlation between CT lung densitometry and visual assessment at $10 \%$ vital capacity (all $p>0.05$ ).

There was a tendency to higher coal worker's pneumoconiosis score of the whole lung with increasing cumulative dust exposure. Cumulative dust exposure of coal miners with a normal thin-section CT ( $n=7)$ was $66 \pm 45 \mathrm{gh} / \mathrm{m}^{3}$ and significantly different $(p<0.05)$ from cumulative dust exposure of coal miners with a more severe abnormal thin-section CT consistent with coal worker's pneumoconiosis (121 \pm 44 $\mathrm{gh} / \mathrm{m}^{3}, \mathrm{n}=17$ ). Cumulative dust exposure of coal miners with a mild abnormal thinsection CT suggestive of coal worker's pneumoconiosis $\left(86 \pm 58 \mathrm{gh} / \mathrm{m}^{3}, \mathrm{n}=22\right)$ differed not significantly from both groups of coal miners. In the whole coal miner population, a moderate correlation was present between the coal worker's pneumoconiosis score of the whole lung and the cumulative dust exposure $(r=0.45, p<0.005)$. However, there was no significant correlation between CT lung densitometry and cumulative dust exposure (all $\mathrm{p}>0.05$ ). 
Table 4. Correllation between Objective and Subjective Parameters of Coal Worker's Pneumoconiosis in 46 Coal Miners al the same Anatomicall Level at $90 \%$ and 10\% Vital Capacity.

\begin{tabular}{|c|c|c|c|}
\hline & $\begin{array}{l}\text { Mean lung density } \\
r\end{array}$ & $\begin{array}{l}\text { Highest } 10 \text { h } \\
\text { percentile } \\
\text { r }\end{array}$ & $\begin{array}{l}\text { Lowest } 10 \text { th } \\
\text { percentile } \\
r\end{array}$ \\
\hline \multicolumn{4}{|l|}{ Upper lung zones } \\
\hline $90 \%$ vital capacity & 0.76 & 0.71 & 0.69 \\
\hline 10\% vital capacity & 0.38 & 0.31 & 0.27 \\
\hline \multicolumn{4}{|l|}{ Lower Iung zones } \\
\hline $90 \%$ wital capactry & 0.53 & 0.46 & 0.45 \\
\hline $10 \%$ wital capacity & 0.28 & 0.26 & 0.27 \\
\hline
\end{tabular}

Note. All correlations are statistically significant $(\mathrm{p}<0.05$; one tailed $)$.

Table 5. Corrdation between Objective and Subjective Parameters of Coal Worker's Pneumoconiosis in T'wo Groups of Coal Miners with Coal Worker's Pneumoconiosis as Assessed with Thin-Section CT at $90 \%$ Vital Capacity.

\begin{tabular}{|c|c|c|c|c|}
\hline \multirow[t]{2}{*}{ CWP score } & \multicolumn{2}{|c|}{$\begin{array}{l}\text { Group } 2 \\
(n=22) \\
0<X \leq 15\end{array}$} & \multicolumn{2}{|c|}{$\begin{array}{l}\text { Gromp } 3 \\
(n=17) \\
>15\end{array}$} \\
\hline & r & $\mathrm{p}$ & $r$ & $\mathrm{p}$ \\
\hline \multicolumn{5}{|l|}{ Upper zanes } \\
\hline Mean lung density & 0.32 & NS. & 0.72 & $<0.005$ \\
\hline Highest 1 oth percentile & 0.35 & N.S. & 0.62 & $<0.001$ \\
\hline Lowesit 10th percentile & 0.17 & N.S. & 0.64 & $<0.05$ \\
\hline \multicolumn{5}{|l|}{ Lower zones } \\
\hline Meinn lung density & 0.11 & N.S. & 0.65 & $<0.001$ \\
\hline Highest roth percentile & .0 .01 & $\mathbb{N}_{.} \mathrm{S}_{\text {. }}$ & 0.58 & $<0.01$ \\
\hline Lowest 10 hercentile & 0.16 & N.S. & 0.56 & $<0.001$ \\
\hline
\end{tabular}

Note. CWP score = coal worker's pneumoconiosis score, N.S. = not significant.

\section{DISCUSSION}

Our study in a coal miner population demonstrates that progression of coal workers pneumoconiosis is associated with increasing attenuation values in both lung zones and at both levels of inspiration. At $90 \%$ vital capacity (VC), good correlations between objective and subjective indicators of coal worker's pneumoconiosis were observed in the upper zones, and moderate correlations in the lower zones of the lungs. At $10 \%$ vital capacity the correlations in both lung zones were poor. The mean lung density was the densitometric parameter which correlated best with visual assessment of thin-section CT. With increasing extent of coal worker's pneumoconiosis, correla- 
tions between CT lung densitometry and visual assessment improved considerably. No significant correlations between $\mathrm{CT}$ lung densitometry and visual assessment were observed in early stages of coal worker's pneumoconiosis.

At both levels of inspiration and in both zones of the lungs we found higher densitometric values with increasing extent of coal worker's pneumoconiosis. At 90\% vital capacity, the densitometric parameters of subjects with a normal and a more severe abnormal thin-section CT consistent with coal worker's pneumoconiosis differed significantly in both lung zones. At 10\% vital capacity a similar densitometric pattern was observed. However, at $10 \%$ vital capacity, differences between mean densitometric parameters in the three groups were less pronounced and never statistically significant. This finding is in contrast to findings observed in patients with chronic obstructive pulmonary disease (COPD) where the analysis of CT slices obtained at $10 \%$ vital capacity was an essential part in the differentiation between patients with COPD and healthy persons $(27,28)$. Lung function impairment in patients with COPD results in air-trapping at lower lung volumes and as a consequence significant diffe-rences in density between COPD patients and healthy volunteers can be observed. No lung function impairment in coal miners and healthy volunteers was seen, nor any difference in lung function between the three subgroups arbitrarily constituted on the basis of the coal worker's pneumoconiosis score of the whole lung.

At increasing extent of coal worker's pneumoconiosis, correlations between CT lung densitometry and visual assessment of thin-section CT improved considerably. Early detection of coal worker's pneumoconiosis would be, in our opinion, the most useful clinical application of CT lung densitometry. Although visual assessment of thin-section CT is considered to be the standard of reference for the depiction of fine parenchymal opacities, it may be difficult in the early stages of coal worker's pneumoconiosis, to differentiate blood vessels on end from micronodular unfiltration at thinsection CT (8). We also encountered difficulties in this group of coal miners in confident assessment of micronodules. Correlations between CT densitometry and visual assessment of thin-section CT in coal miners with a mild abnormal thin-section CIT suggestive of coal worker's pneumoconiosis were poor. The present findings are consistent with previous reports showing that correlation between antemortem radiographic findings in coal worker's pneumoconiosis was substantially better for the more severe categories of disease $(30,31)$.

On the basis of recent work we think that our scan technique and data analysis are amenable for improvements. A sliding-thin-slab maximal intensity projection technique was reported to detect more accurately mild forms of micronodular infiltration (31). Further, density measurements are strongly dependent on section thickness and reconstruction kernel. For instance, thin-section densitometry using 1-mm sections in combination with high resolution kernels was not recommended for determining CT number histogram related parameters other than average density (32). In the pres- 
ent study the mean lung density was the densitometric parameter which correlated best with visual assessment of thin-section $\mathrm{CT}$, but the correlation of the other two densitometric parameters might improve when density resolution is better.

Cr densitometry is not likely to be able to distinguish coal worker's pneumoconiosis from most other conditions that cause increased lung density. A shift towards higher densitometric values in not uniquely due to coal worker's pneumoconiosis since also other interstitial and alveolar lung diseases (33-35), panbronchiolitis (36), and postirradiation effects (37) are manifested by similar changes in physical density. For an ultimate diagnosis of coal worker's pneumoconiosis a key position remains to be reserved for visual assessment of thin-section CT scans.

The issue of emphysema in coal miners is controversial and subject to debate in the literature. A necropsy study has shown that emphysema is more common in coal worker"s pneumoconiosis than in a population of non-dust exposed controls (38). Focal emphysema is commonly found in preumoconiosis with p-type changes and is related to dust exposure $(39,40)$. Other authors have concluded that progressive massive fibrosis is a crucial factor in determining the presence of emphysema (41), and that in simple coal worker's pneumoconiosis, emphysema is hardly seen $(9,42)$. If pneumoconiosis with p-type changes and emplyysema are associated it is expected that with progression of both disorders, the highest 10 th percentile increases, and the lowest 10 th percentile of the frequency distribution decreases in attenuation. Both changes would than nullify mean lung density changes. However, all the three parameters showed comparable changes in density with progression of coal worker's pneumoconiosis.

The overall extent of coal worker's pneumoconiosis in the lung, visually assessed at thin - section CT was found to be related to exposure to respirable dust. There was no significant correlation between cumulative dust exposure and the various densitometric parameters at both levels of inspiration. A possible explanation for this difference is the fact that the visual coal worker's pneumoconiosis score is based on the sum of the resulks of four CT slices whereas our densitometric results are based on the analysis of one CT slice only, which could give rise to sample error and statistically less robust estimates. A spiral volumetric CI technique would enable anatomically contiguous scanning and analysis of scans of the whole lung will probably more reliable reproduce coal worker"s pneumoconiosis changes in the lung than the data of one single CT slice.

Longitudinal studies have demonstrated a significant negative relation between cumulative dust exposure and forced expiratory volume in one second (FEV $)(43-$ 45). Loss of $\mathrm{FEV}_{1}$ is independent of the presence of pneumoconiosis $(43,44)$. In our study $F_{E V}$ and $K_{C O}$ values tended to decrease with cumulative dust exposure and mean coal worker's pneumoconiosis scores. However, all values are still normal and $\mathrm{FEV}_{1}$ differences were not statistically significant, probably due to the relatively small study population and the relatively low cumulative dust exposure as well. $K_{C o}$ was the 
only pulmonary function test which was, although in the normal range, significantly lower in workers with a more severely abnormal thin-section CT consistent with coal worker's pneumoconiosis than in subjects with a normal thin-section CT.

In conclusion, for the diagnosis of coal worker's pneumoconiosis, a history of extensive coal dust exposure, clinical and functional findings as well as nodular opacities assessed at chest radiography are required. CT scans obtained at $90 \% \mathrm{VC}$ in the upper zones of the lungs are the best candidates for lung densitometric measurements in coal worker's pneumoconiosis. CT lung densitometry correlates well with visual assessment of thin-section CT of the lung provided that CT slices are obtained at $90 \%$ vital capacity. Further improvements of the technique are possible. Numbers of participating coal miners in this study were relatively small and further prospective studies need to be performed to assess the diagnostic accuracy and role of $\mathrm{CT}$ densitometry in coal worker's pneumoconiosis.

\section{REFERENCES}

1. International Labour Office (1980). Guidelines for the use of ILO international classification of radiographs of pneumoconiosis, rev ed. International Labour Office Occupational and Heath safety No. 22 (REV 1980) Geneva: International Labour Office

2. Rockoff SD, Schwartz A. Roentgenographic underestimation of early asbestosis by International organization Classification. Chest 1988; 93:1088-1099.

3. Vallyathan V, Brower PS, Green FHY, Attield MD. Radiographic and pathologic comelation of coal worker's pneumoconiosis. Am J Respir Crit Care Med 1996; 154: 741-748.

4. Nakala $H_{1}$, Kimoto T, Nakayama T, Kido M, Miyazaki N, Harada S. Diffuse peripheral lung disease: evaluation of high resolution computed tomography, Radiology 1985; 157:181-185.

5. Müller NL, Miller RR, Webb WR, Evans KG, Ostrow DN. Fibrosing alveolitis: Cl-pathologic correlation. Radiology $1986 ; 160: 585-588$.

6. Mathieson JR, MayoJR, Staples CA, Muller NL. Chronic difuse infiltrative lung discase: comparison of diagnostic accuracy of CT and chest radiography. Radiology $1989 ; 171: 111-116$.

7. Staples CA, Gamsu G. Sue Ray C. Webb RW. High resolution contputed tomograplyy and lung function in asbestos-exposed workers with nomal chest radiographs. An Rew Respir Dis 1989, 139:15021508.

8. Remy-Jardin M, Degreef JM, Beuscart R, Voisin C, Remy J. Coall worker's pneumoconiosis: CT" assessment in exposed workers and conrelation with radiographic findings. Radiology $1990 ; 177.363$ 371.

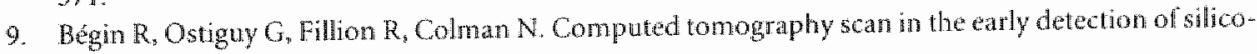
sils. Am Rev Respir Dis 1991: 144:697-705.

10. Levi C, Gray IE, MeCullough EC, Hattery RR. Variability of CT numbers as absolute walues. A AR 1982: $139: 443-447$

11. Zerhoun EA, Spivey IF, Morgan RH, Leo FP, Stitik EP, Siegeman SS. Factors influencing quantitue CT measurements of solitary pulnonary nodules. I Comput Assist Tomogr $1982 ; 6: 1075 w 1087$.

12. MeCullough EC, Morin RL. CT-number variability in thorawic geometry. AIR 1982; $14: 135-1.40$.

13. Kemerink GJ, Lamers R35, Thelissen GPR, van Engelshoven IMA. Scanner conformily in CT densitometry of the lungs. Radiology 1995, 197:749-752. 
14. Kemerink G], Lamers RIS, Thelissen GRP, van Engelshoven MMA. CT-densitometry of the lungs: scanner performance. I Comput Assist Tonogr 1996; 20:24-33.

15. Maller NL, Staples CA, Miller RR, Abboud RT. "Density Mask": an objective method to quantitate emphysema using computed tomography. Chest 1988; 94:782-787.

16. Gould GA, MacNee $W$, McLean $A_{n}$ et al. CT density measurements of lung density in life can quantitate distal airspace enlargement: an essential defining feature if human emphysema. Am Rev Respir Dis $1988 ; 137.380-392$.

17. Gevenois PA, de Martelaer V, De Vuyst P, Zamen J, Yernault $J C$. Comparison of computed density and macroscopic morphometry in pulmonary emphysema. Am J Respir Crit Care Med 1995; 151:653-657.

18. Hartley $P G$, Galwin JR, Hunninghake $G W$, et al. High-resolution $C T$ derived measures of lung density are valid indexes of interstitial lung disease. I Appl Physiol 1994; 76:271-272.

19. Beinert T, Behr J, Mehnert F, et al. Spirometrically controlled quantitative CT for assessing diffuse parenchymal lung disease. ] Comput Assist Tomogr 1995; 19:924-931.

20. Kalender WA, Rienmüller R, Seissler W, Behr J, Welke M, Fichte H. Measurement of pulmonary parenchymal attenuation: use of spirometric gating with quantitative CT. Radiology 1990; 175:265-268.

21. Schins RPF, Borm PJA. Epidemiological evaluation of monocyte-a release as an exposure marker in pneumoconiosis: a five year follow up study among coal workers. Occup Environ Med 1995; $52: 441$. 450 .

22. Rienmuller RK, Behr J, Kalender WA, et al. Standardized quantitative high resolution CT in lung disease. J Comput Assist Tomogr 1991; 15:742-749.

23. Borm PJA, Palmen N, Engeten IJM, Buurman WA. Spontaneous and stimulated rellease of tumor necrosis factor (TNF)- $\alpha$, from blood monocytes of miners with coal workers" pneumoconiosis. Am Rev Respir Dis 1988; 138:1589-1594.

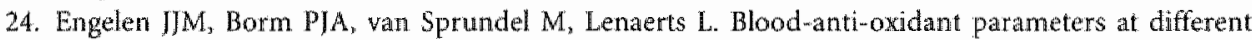
stages of pneurnoconiosis. Env Health Persp 1990; 84:165-172.

25. Quanjer PH. Standardized lung function testing. Bull Eur Physiopathol Respir 1983; 19.7 44.

26. Foster WL, Pratt PC, Roggli VL, Godwin JD, Halvorsen RA, Putman CE. Centrilobullar emphysema: CT"-pathologic correlation. Radiology 1986; 159:835m837.

27. Lamers RIS, Thelissen GRP, Kessels AG, Wouters EFM, van Engelshoven JMA. Chronic obstructive pulmonary disease: evaluation with spirometrically controlled CT lung densitometry. Radiology 1994: 193:109-113.

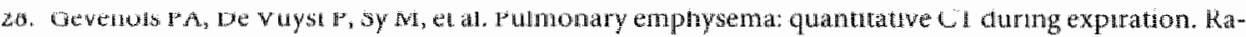
diology 1996; 199:825-829.

29. Caplan A. Correlation of radiological category with lung pathology in coal workers' pneumoconiosis. BrJ Radiol 1961: 19:171-179.

30. Fernie IM, Ruckley VA. Coalworkers' pneumoconiosis: correlation between opacity profusion and number and type of dust lesions with special reference to opacity type. Br I Ind Med 1987; 44:273-277.

31. Remy-Jardin $M$, Remy I. Artaud D, Deschildre $F$, Duhamel A. Diffuse infiltrative lung disease: clinical value of sliding thin-slab maximum intensity projections CT scans in the detection of mild micronodular patterns. Radiology $1996 ; 200: 333-339$.

32. Kemerink GJ, Kruize HH, Lamers RJS, van Engelshoven JMA. Density resolution in quantitative computed tomography of foam and lung. Med Phys 1996;23;1697-1708.

33. Gilman MI, Laurens RG, Somogyi IW, Honig EG. CT attenuation values of lung density in sarcoidosis. J Comput Assist Tomogr 1983; 7:407-410.

34. Bellamy EA, Nicholas D, Husband IE. Quantitative assessment of lung damage due to bleomycin using computed tomography. Br I Radiol 1987; 60:1205-1209.

35. Hedlund LW, Effman EL., Bates WM, Beck IW, Goulding PL, Putman CE. Pulmonary edena: a CT study of regional changes in lung density following oleic acid injury. J Comput Assist Tomogr 1982; $6: 939-946$. 
36. Maruta $\mathrm{K}$, Itoh $\mathrm{H}$, Senda $M$, et all Stratified impairment of pulmonary ventilation in 'diffuse panbronchiolitis": PET and CT studies. I Comput Assist Tomogr 1989; 13:48-53.

37. Van Dyk J, Hill RP. Post-irradiation lung density changes as measured by computerized tomography. Int J Radiat Oncol Biol Phys 1983; $9: 847-852$.

38. Cockcroft A, Wagner JC, Ryder R, Seal RME, Lyons JP, Andersson A. Post-mortem studies of emphysema in coal workers and non-coal workers. Lancet 1982; ii: 600-603.

39. Leigh 1, Outhred KG, McKenzy HI, Glick M, Wiles AN. Quantified pathology of emphysema, pneumoconiosis, and chronic bronchitis in coal workers. Br J Ind Med 1983; 40:258-263.

40. Akira M, Higashihara T, Yokoyama K, et al. Radiographic type p pneumoconiosis: high-resolution CT. Radiology 1989; 171: 117-123.

41. Ruckley VA, Gauld SJ, Chapman IS. Emphysema and dust exposure in a group of coal workers. Am Rew Respir Dis 1984; 129:528-532.

42. Kinsella M, Müller $N_{0}$ Vedal S, Staples C, Abboud RT, Chan-Yeung M. Emphysetna in silicosis: a comparison of smokers with nonsmokers using pulmonary function testing and computed tomography, Am Rev Respir Dis 1990; 141:1497-1500.

43. Soutar CA, Hurley JF. Relation between dust exposure and lung function in miners and ex-miners. $\mathrm{Br}$ J Ind Med 1996; 43:307-320.

44. Attfield $M D_{n}$ Hodous TK. Pulmonary function of U.S. coal miners related to dust exposure estimates. Am Rev Respir Dis 1992; 145:605 609.

45. Oxman AD, Muir DCF, Shannon HS, Stock SR, Hnizdo E, Lange HJ. Occcupational dust exposure and chronic obstructive pulmonary disease: a systematic overview of the evidence. Am Rev Respir Dis $1993 ; 148: 38 m 48$. 



\section{Summary and conclusions}

Since its introduction, computed tomography (CT) densitometry has been promoted as a useful method for determining the regional and global density of the lung. The Xray atte-nuation of soft tissue as measured by CT has a linear relationship to the soft tissue density $\left(\mathrm{g}^{\prime} \mathrm{cm}^{3}\right)$ and therefore densitometry of the lungs has the potential to provide non-invasively objective information about lung density, its spatial distribution and its changes over time. The aim of this thesis is to determine the inter-and intrascanner conformity of various CT scanners for lung densitometry purposes, to standardize the technique of post-processing and to determine the reproducibility of this method in a clinical setting. Futhermore we applied CT lung densitometry to clinical studies of chronic obstructive pulmonary disease (COPD) and silicosis pulmonum.

Chapter 2 gives a brief overview of several aspects of chronic obstructive pulmonary disease (COPD): definition, pathologic characterization, clinical features, pulmonary function tests, and radiological diagnosis. COPD severity can be staged on the basis of the degree of airflow obstruction. The test is not suitable to distinguish between chronic bronchitis and emphysema. Disturbance of gas exchange manifested by reduced single-breath carbon monoxide diffusing capacity ( $\mathrm{DL}_{\mathrm{CO}}$ ), have been proposed as the most accurate and specific pulmonary function tests for pulmonary emphysema. High resolution CT (HRCT) has also proven to be an accurate noninvasive technique to diagnose emphysema in vivo, and high correlations between visual $\mathrm{CT}$ emphysema scores and pathological grades have been reported $(0.57-0.91)$.

Comparison of densitometric results obtained on different scanners has proved difficult for various reasons. Most CT scanners were not optimized for lowattenuation studies and nearly all densitometric studies published so far were deficient in their attention to analysis protocols and applied methodology. An additional problem is the influence of level of inspiration on lung density. Changes by more than a factor of two between full inspiration and expiration are possible. Therefore, control of the level of inspiration is mandatory to obtain reproducible results. Another problem is that the numeric analysis of CT data of lung tissue is operator dependent and very time consuming. Support by an automatic evaluation method is necessary to achieve high precision and to reduce operator's influence. 
We assessed the performance of our CT scanner at air density. It proved to have a stability within $1 \mathrm{HU}$ and a reproducibility for density of lung tissue within 2-3\%. Additionally we implemented a spirometric CT triggering technique, as described by Kalender and coworkers, to get the possibility to scan at defined levels of inspiration. To ensure reproducibility and objectivity, an automatic evaluation procedure for CT densitometry of the lungs has been developed. This computer program, using a segmentation threshold in a contour tracking algorithm, isolates both lungs automatically after which all relewant CT parameters are calculated.

Chapter 3 gives an overview of the literature of CT densitometry of the lungs and describes the CT protocol, the technique of respiratory gating of the CT scanner and dosimetrical aspects.

In chapter 4 the issue of conformity between the various scanners on the market is addressed. A systematic comparison of lung densitometry as calculated with six CT scanners was performed; GE Highlight, Picker PQ-2000, Philips scanners SR 7000 and $\mathrm{LX}$, and two Siemens Somatom Plus systems. All CT scanners had an acceptable calibration of water density. The scanners were well designed with respect to CT number sensitivity to reconstruction filter, zoom factor, table height and slice thickness, with the exception of the two Philips systems in regard to sensitivity to slice thickness. CT numbers from the GE Highlight and Picker PQ-2000 scanner showed some sensitivity to phantom size and composition. The Siemens Somatom Plus systems were well calibrated and not very sensitive to phantom size or composition. After correcting for poor air calibration the conformity of all scanners in the low density range was fair: at a density of about $100 \mathrm{~kg} / \mathrm{m}^{3}$ the standard deviation of the average over all scanners is less than $3 \mathrm{HU}$, and the maximum observed inter-scanner difference is $7 \mathrm{HU}$.

In chapter 5 a contribution is given to optimize the method of image analysis. An image analysis program uses a segmentation threshold which discriminates soft tissue from lung by fast contour tracking. In addition, outer pixels that are still affected by the thoracic wall or larger vessels are excluded by shrinking the contour to obtain truly lung parenchyma related data. This process is called "erosion". Subsequently, CT number histograms of both lungs are detemined and the densitometric parameters are calculated automatically. Changing segmentation threshold and number of erosions affect strongly the number of pixels that belong to the high density part of the CT number histogram of the lung.

Most previous authors paid little attention to the analysis protocol and were not aware of the large differences in densitometric parameters which are introduced by changing segnentation threshold and numbers of erosions. The segmentation procedure is usually poorly described and often there is no mention whether erosions have been applied. A low threshold is well suited for segmentation along thin septa but a too low threshold can lead to exclusion of parts of the lungs. For comparability of results, the same evaluation protocols should be applied. From our experiments it was con- 
cluded that for densitometry a segmentation threshold of $-400 \mathrm{HU}$ combined with two erosions should be applied. For volumetry, the same threshold combined with zero erosions should be adequate.

In chapter 6 the reproducibility of spirometrically controlled CT lung densitometry at defined levels of inspiration in a clinical setting was assessed. CT sections were obtained on two separate days from 20 hospitalized patients. Various lung density parameters, reflecting the shape of the CT number histogram were calculated and results of corresponding CT sections were compared. The most reproducible CT lung density measurements were obtained at $90 \%$ vital capacity. At this level of inspiration, reproducibility was of the order of $3-14 \mathrm{HU}$. At $10 \%$ vital capacity, however, reproducibility was worse by a factor of three. If CT scans at residual volume are used for diagnosis and follow-up, respiratory control is mandatory. Reproducibility of lung density measurements was not influenced by severe respiratory insufficiency.

In chapter $7 \mathrm{CT}$ lung densitometry results at defined levels of inspiration from 3 groups of 20 individuals each were compared: a group consisting of patients who fulfilled the functional criteria of emphysema; a group who met the clinical and functional criteria of chronic bronchitis, and a group of persons who had no pulmonary symptoms and normal results of lung function tests. This study confirmed the value of spirometrically controlled CT densitometry in the quantitative assessment of lung density at selected levels of inspiration. Three densitometric parameters, namely mean lung density, percentage of pixels in the -910 to $-1024 \mathrm{HU}$ range (pixel index), and the cut-off point that defines the lowest 10th percentile of the frequency distribution of HU numbers, were compared. Regardless of the level of inspiration, significant differences were found between patients who had emphysema, healthy persons and patients who had chronic bronchitis. Differences between healthy subjects and patients with chronic bronchitis were only significant at $10 \%$ vital capacity. On the basis of these results, we concluded that two sections obtained at the same level - one at $90 \%$ vital capacity and one at $10 \%$ vital capacity - are sufficient for the classification of subjects into one of the three groups.

In chapter 8 we assessed the association of emphysema with airflow limitation in patients with advanced COPD, and studied the point of debate whether DLCO can be used as an indicator for emphysema in advanced COPD. In a study of 100 paticnts with COPD we used high resolution CT of the lungs in combination with CT lung densitometry, as the gold standard for the diagnosis of emphysema and for assessment of its severity. Using a visual grading method, CT-scored significant emphysema was found mainly in patients with stage II or stage III COPD (FEV $<50 \%$ predicted). Lung density measurements confirmed visual observations. With progression of airHow limitation, the lung density in patients with COPD obtained at $90 \%$ vital capacity and at $10 \%$ vital capacity decreased as expected. Results suggest a distinct presence of emphysema in patients with advanced COPD. The DLCO proved to be a useful test to 
separate emphysmea from chronic bronchitis types of COPD. The vast majority of patients with COPD (93\%) and a DLCO \% predicted of less than $50 \%$ showed emphysematous changes at $\mathrm{CT}$, and $56 \%$ of these patients suffered from significant emphysema. In contrast with this, only $23 \%$ of patients with COPD and $D L_{C O} \%$ predicted of equal to or more than 50 showed emphysema on CT, and only a small minority of patients (4\%) showed significant emphysematous parenchymal changes on CT. We found a good correlation between DLCo and the severity of emphysema independent of the degree of airflow limitation. However in patients with $D L_{C o}$ less than $50 \%$ predicted, the correlation between $\mathrm{DL} C O$ and the severity of emphysema was poor. DLCo measurements cannot be relied on for quantitative assessment of severity and extent of emphysema, but $D L_{C O}$ measurements are valuable for selection of patients for radiologic emphysema assessment.

The usefulness of CT lung densitometry in the assessment of interstitial lung disease was investigated in chapter 9 . Thin-section CT scans were obtained of a group of 66 persons consisting of 35 coal miners with a normal chest radiograph, 11 coal miners with a chest radiograph showing nodular opacities consistent with coal worker's pneumoconiosis, and 20 healthy volunteers. All scans were visually assessed for the presence and extent of coal worker's pneumoconiosis. In addition, spirometrically gated CT sections of the upper and the lower zones of the lungs were obtained at $90 \%$ and $10 \%$ vital capacity. Based on results of visual assessment, subjects were divided into three subsets. Densitometric parameters of coal worker's pneumoconiosis obtained from the three groups were compared and correlated with results of visual assessment of CT scans. Progression of coal worker's pneumoconiosis was associated with increasing attenuation values in both lung zones and at both levels of inspiration. Densitometric parameters between groups differed significantly only in the upper zones at $90 \%$ vital capacity. CT densitometry correlated well with visual assessment of thin-section CT of the lung provided that the CT sections were obtained at $90 \%$ vital capacity. At 10\% vital capacity correlations were always poor. CT scans obtained at $90 \%$ vital capacity in the upper zones of the lungs are the best candidates for lung densitometric measurements in coal worker's pneumoconiosis.

\section{In conclusion}

Modern CT scanners that are properly calibrated are well suited for densitometric studies of the lung, but to attain generally comparable results the same scan and analysis protocols should be applied. For the study of the lungs at intermediate levels of inspiration, gated triggering of the CT scanner is required. Reproducibility of densitometric results using this technique was shown to be satisfactory. Densitometry proved to be useful in the assessment of pulmonary emphysema and might also be helpful in the diagnosis and follow-up of interstitial lung disease 


\section{Future developments}

The development of very fast volume scanning makes possible the investigation of the whole lung within a single breath-hold period. Using this technique it is likely that total lung mass in viwo can be determined much more accurately than was previously possible. With suitable software for the analysis of the large datasets obtained, volume scanning might then find application in the field of lung volume reduction surgery and in longitudinal studies of various forms of lung disease.

Surgical resection of nonfunctioning areas of lung tissue - lung volume reduction surgery- is emerging as a promising option for the treatment of selected patients with severe debilitating emphysema. Volumetric CT densitometry may be helpful in selecting candidates who will benefit from lung volume reduction surgery. From a surgical point of view, assessment of the relative severity and location of emphysematous lung may be useful so that the most emphysematous section of the lung can be removed. In conjunction with clinical findings and pulmonary function tests, these densitometric data will improve our understanding of the full mechanisms responsible for the improvement after lung volume reduction surgery as well as of the long-term outcome.

CT can be used to quantify the progression of the pathology in emphysema. Longitudinal studies of lung mass might be very rewarding if combined with metabolic studies. There is a growing interest in the relationship between the nutritional status and lung structure. Food deprivation alters the architecture of terminal air spaces in a manner similar to that which occurs in emphysema. Studies mainly performed in animals have repeatedly shown that lung weight is decreased in starvation. A substantial number of patients suffering from COPD also experience gradual and significant weight loss during the course of their illness yet the potential effect on lung weight is unknown. The significance of in vivo determination of lung density could be of particular interest from the point of view of understanding the pathophy-siology of starvation-induced changes in pulnonary structure, but also to evaluate the effectiveness of therapeutic strategies to improve nutritional status. 



\section{Samenvatting}

Al sedert de introductie van computertomografie (CT) is densitometrie gebruikt om op niet-invasieve wijze de regionale en gemiddelde dichtheid van de longen te bepalen. CT densitometrie is gebaseerd op het feit dat de door de CT scanner in wekedelen gemeten werzwakking van de röntgenbundel een lineaire relatie kent met de dichtheid $\left(\mathrm{g} / \mathrm{cm}^{3}\right)$. De techniek biedt de mogelijkheid om objectieve informatie te verschaffen omtrent de dichtheid van de longen en eventuele veranderingen daarin.

Doelen van dit proefschrift zijn: het vergelijken van de prestaties van verschillende CT scanners op het gebied van longdensitometrie, het bepalen van de variabiliteit van één en dezelfde scanner, het leveren van een bijdrage aan de standaardisering van zowel de scantechniek als de post-processing van CT beelden, het bepalen van de reproduceerbaarheid van spirometrisch gecontroleerde longdichtheidsmetingen in de dagelijkse praktijk, en tenslotte het toepassen van spirometrisch gecontroleerde CT triggering in enkele pulmonologische klinische studies.

In hoofdstuk 2 worden verscheidene aspecten van chronisch obstructief longlijden (COPD) belicht: definities, pathologische karakterisering, klinisch beeld, longfunctieonderzoek en radiologisch onderzoek. Een sterk verlaagde expiratiesnelheid is een spirometrisch ventilatoire bevinding wijzend op bronchusobstructie, terwijl de diffusiecapaciteit van koolmonoxyde (DL $\mathrm{CO}_{\mathrm{O}}$ ) meer specifieke informatie geeft ontrent het al dan niet aanwezig zijn van emfyseem.

Hoge resolutie CT wordt als de meest betrouwbare onderzoeksmethode beschouwd om emfyseem op een niet-invasieve manier in vivo te diagnostiseren en te kwantificeren. Correlaties tussen visuele CT emfyseemscores en pathologische emfyseemscores liggen in de orde van grootte wan 0.57 tot 0.91 .

In het verleden is vergelijking van densitometrische resultaten, verkregen met verschillende CT scanners, om werschillende redenen niet mogelijk gebleken. De meetresultaten van veel CT scanners bleken in lage-dichtheidstudies onvoldoende nauwkeurig en daarnaast werd in vrijwel alle tot op heden gepubliceerde CT densitometrische studies geen aandacht geschonken aan acquisitie- en analyseprotocollen. Een ander belangrijk probleem was de invloed van de mate van inspiratie op de longdichtheid: veranderingen in de orde van grootte van een factor 2 tussen inspiratie en expiratie zijn mogelijk. Controle van het inspiratieniveau tijdens het scannen is dus een eerste vereiste voor reproduceerbaarheid van metingen. Ook was de handmatig 
uitgevoerde analyse van de CT data afhankelijk van de onderzoeker. Bovendien was deze analyse zeer tijdrovend. Automatische evaluatieprogrammatuur lijkt noodzakelijk om binnen acceptabele tijd een hoge mate van precisie te bereiken en om de invloed van de onderzoeker op het uiteindelijke meetresultaat te elimineren.

We hebben de prestaties van onze CT scanner ten aanzien van luchtdichtheidmetingen bepaald. Het systeem bleek correcte resultaten te leveren (CT-waarde: $1000 \pm 1$ Hounsfield Unit, HU) en toonde over een periode van meer dan een jaar een verloop wan hooguit 1 HU. De reproduceerbaarheid wan metingen aan longweefsel lag in de orde van 2 tot $3 \%$.

Daarnaast hebben wij een door Kalender en medewerkers beschreven spirometrische 'triggering techniek' geïmplementeerd, teneinde de mogelijkheid te verkrijgen om op vooraf in te stellen inspiratieniveaus te scannen.

Om objectiviteit van de analyse van CT data te waarborgen werd speciale, in hoge mate geautomatiseerde evaluatieprogrammatuur ontwikkeld ten behoeve van CT longdensitometrie. Deze programmatuur maakt gebruik van een algoritme dat automatisch de longwand zoekt, en deze op een in te stellen CT waarde (de segmentatiedrempel) volgt zodat het longparenchym van het andere thoraxweefsel gescheiden wordt. Na deze automatische segmentering, die met de hand nog gecorrigeerd kan worden, worden alle relevante densitometrische parameters berekend.

In hoofdstuk 3 wordt een kort literatuuroverzicht gegeven inzake CT longdensitometrie, gevolgd door een beschrijving van het scan protocol, de spirometrische 'CT triggering techniek' en de wijze waarop de analyse van CT data geschiedt. Tenslotte komen dosimetrische aspecten van het gebruikte CT scanprotocol aan de orde

In hoofdstuk 4 worden de densitometrische prestaties van een zestal CT scanners vergeleken. In het onderzoek zijn betrokken: GE Highlight, Picker PQ-2000, de Philips scanners SR 7000 en LX, en 2 Siemens Somatom Plus systemen. Alle CT scanners toonden een acceptabele kalibratie voor water, terwijl de kalibratie voor lucht voor sommige scanners enigszins te wensen overliet. De scanners konden de toets der kritiek wat betreft de gevoeligheid voor reconstructiefilter, vergrotingsfactor, tafelhoogte en snedeclikte goed doorstaan, met uitzondering van de twee Philips systemen die een grote gevoeligheid voor coupedikte aan de dag legden. De CT- waarden van de GE Highlight en Picker PQ-2000 scanner bleken enigszins gevoelig voor fantoomgrootte en -samenstelling. Na correctie van de luchtkalibratie waren de meetresultaten van de diverse scanners vergelijkbaar. Voor dichtheden rond $0.1 \mathrm{~g} / \mathrm{cm}^{3}$ bedroeg de standaarddeviatie van de resultaten van alle scanners minder dan $3 \mathrm{HU}$, terwijl het maxjmale gemeten verschil tussen twee scanners 7 HU bedroeg.

In hoofdstuk 5 wordt een bijdrage geleverd aan de optimalisatie van de analysemethodieken van CT data. Er is een computerprogramma ontwikkeld waarin een segmentatiedrempel kan worden ingesteld die in een contourdetectiealgoritme wordt gebruikt om longparenchym te scheiden wan het de longen omhullende wekeweefsel. 
Na segmentatie kunnen de buitenste pixels, die mogelijk nog beinvloed zijn door de thoraxwand of de grote vaten, geëxcludeerd worden om data te verkrijgen die louter met longparenchym gerelateerd zijn. Het excluderen van de buitenste pixels, dus het 'krimpen' van de contour, heet erosie. Vervolgens worden automatisch CT-waarden histogrammen van beide longen berekend, en hieruit alle gewenste densitometrische parameters.

Veranderingen in segmentatiedrempel en aantal erosies beinnvloeden wezenlijk het aantal pixels dat behoort tot het hoge-dichtheidsdeel van het CT-waarden histogram. In het verleden hebben de meeste auteurs aan analyseprotocollen weinig aandacht geschonken. De segmentatieprocedure is gewoonlijk slecht beschreven en vaak wordt zelfs niet vermeld of, dan wel hoeveel, erosies er zijn toegepast. Een lage segmentatiedrempel is weliswaar geschikt om nog succesvol langs dunne septae te segmenteren, maar een te lage segmentatiedrempel kan resulteren in uitsluiting van longparenchym. Om vergelijkbare resultaten te verkrijgen dienen dezelfde acquisitie- en evaluatieprotocollen te worden toegepast. Uit onze experimenten kan worden geconcludeerd dat voor longdensitometrie een segmentatiedrempel van $-400 \mathrm{HU}$, gecombineerd met 2 erosies, meestal optimaal is. Voor volumetrie is dezelfde segmentatiedrempel, maar nu gecombineerd met nul erosies, het meest geschikt.

In hoofdstuk 6 wordt bestudeerd hoe het met de reproduceerbaarheid van spirometrisch gecontroleerde CT densitometrie gesteld is in de dagelijkse praktijk. Op twee opeenvolgende dagen werden op twee spirometrisch gecontroleerde inspiratieniveaus (90\% en 10\% van de inspiratoire vitale capaciteit) bij 20 gehospitaliseerde patiënten CT opnames gemaakt van zowel de boven- als de ondervelden van de long. Densitometrische parameters, die de vorm van het CT-waarden histogram reflecteerden, werden berekend en vergeleken voor corresponderende CT coupes. De meetresultaten verkregen op $90 \%$ van de vitale capaciteit bleken het beste reproduceerbaar te zijn, met gevonden verschillen in de orde van grootte van 3-14 HU. De reproduceerbaarheid was een factor 3 slechter indien werd gescand op $10 \%$ van de vitale capaciteit. Gcconcludeerd werd, dat als $\mathrm{CT}$ scans op residuaal volume nodig zijn voor diagnostick of het wervolgen van respiratoire aandoeningen, deze onder spirometrische controle uitgevoerd dienen te worden. De reproduceerbaarheid van longdichtheidsmetingen bleek niet te worden beïnvloed door de ernst van de respiratoire insufficiëntie van patiënten.

In hoofdstuk 7 werden densitometrische meetresultaten vergeleken afkomstig van 3 groepen personen, waarbij elke groep bestond uit 20 individuen. Eén groep was samengesteld uit patiènten die voldeden aan de functionele criteria van emfyseem, de tweede groep uit patiënten die voldeden aan de klinische en functionele criteria van chronische bronchitis, en de derde groep uit personen zonder pulmonale symptomen en met een normaal longfunctieonderzoek. De CT scans werden wederom spirometrisch getriggerd op $90 \%$ en $10 \%$ van de inspiratoire vitale capaciteit in zowel de 
boven- als de ondervelden. Deze studie bevestigde de waarde van spirometrisch gecontroleerde CT densitometrie in de kwantitatieve vaststelling van longdichtheid op vastgestelde inspiratieniveaus. Ongeacht het niveau van inspiratie werden significante verschillen in dichtheid gevonden tussen patiënten met emfyseem enerzijds, en patiënten met chronische bronchitis en gezonde personen anderzijds. De longdichtheid van gezonde personen en patiënten met chronische bronchitis verschilde alleen significant op $10 \%$ van de vitale capaciteit. De gemiddelde veranderingen in densitometrische parameters tussen 90 en $10 \%$ vitale capaciteit waren bij gezonde personen aanzienlijk groter dan bij patiënten met COPD. Op basis van deze resultaten hebben wij geconcludeerd dat het goed mogelijk is om op basis van de meetresultaten van twee op het zelfde scanniveau verkregen CT sneden, éen op 90\% en één op $10 \%$ van de vitale capaciteit, personen in te delen bij één van deze 3 groepen.

In hoofdstuk 8 werd bij een groep van 100 patiènten met COPD onderzocht of emfyseem geassocieerd is met luchtwegobstructie. Daarnaast werd nagegaan of $\mathrm{DL}_{\mathrm{CO}}$ metingen gebruikt kunnen worden als indicator voor emfyseem. De mate van emfyseem werd met behulp van hoge-resolutie $\mathrm{CT}$ en een visueel graderingssysteem vastgesteld. De resultaten suggereerden een uitgesproken aanwezigheid van emfyseem in patiënten met gevorderd COPD. Significant emfyseem werd voornamelijk aangetroffen bij patiënten met stadium II of III COPD ( $\mathrm{FEV}_{1}$ kleiner dan $50 \%$ van de gemiddelde normale waarde). Visuele observaties werden door longdichtheidsmetingen bevestigd. De dichtheid van de longen nam met progressie van luchtwegobstructie significant af, zowel in scans op $90 \%$ als $10 \%$ van de inspiratoire vitale capaciteit.

$\mathrm{DL}_{\mathrm{CO}}$ bleek bij patiënten met COPD een nuttige longfunctietest te zijn om emfyseem te onderscheiden van chronische bronchitis. De overgrote meerderheid van patiënten met COPD (93\%) en een $\mathrm{DL}_{\mathrm{C}}$ kleiner dan $50 \%$ van de gemiddelde normale waarde, toonde enfysemateuze veranderingen op $C T$, en $56 \%$ van deze patienten leden aarn ernstig emfyseem. Daarentegen vertoonden slechts $23 \%$ van de patiënten met COPD, en een DLCO groter of gelijk dan $50 \%$ van de gemiddelde normale waarde, emfysemateuze veranderingen, waarvan bij een kleine minderheid (4\%) ernstige emfysemateuze veranderingen werden vastgesteld. Er bestond een goede correlatie tussen $\mathrm{DL}_{\mathrm{CO}}$ en de ernst van emfyseem, onathankelijk van de mate van luchtwegobstructie. Daarentegen werd bij patiënten met een $\mathrm{DL}_{\mathrm{CO}}$ kleiner dan $50 \%$ van de gemiddelde normale waarde geen verband aangetoond tussen $\mathrm{DL}_{\mathrm{CO}}$ en de ernst van emfyseem. DLCo metingen zijn dus nuttig om emfyseem bij patiënten met COPD vast te stellen, mar voor emfyseernkwantificering zijn ze niet geschikt. Het is aan te bevelen om bij patiënten met $\mathrm{DL}_{\mathrm{CO}}$ waarden kleiner dan $50 \%$ van de gemiddelde normale waarde de ernst van emfyseem vast te stellen met aanvullende HRCT.

Het nut van CT densitometrie bij de vaststelling van interstitiële longziekten, waarvan stoflongen er én is, was onderwerp van studie in hoofdstuk 9 . Van een groep van 66 personen, bestaande uit 35 mijnwerkers met een normale thoraxfoto, 11 mijnwer- 
kers met een thoraxfoto die nodulaire afwijkingen vertoonde wijzend op stoflongen, en 20 gezonde vrijwilligers werden 5 hoge-resolutie CT scans gemakt. Alle scans werden visueel beoordeeld op de aanwezigheid en uitgebreidheid van interstitiële noduli. Vervolgens werden van iedere persoon spirometrisch gecontroleerde CT sneden van de boven- en de ondervelden gemaakt op respectievelijk $90 \%$ en $10 \%$ van de inspiratoire vitale capaciteit. Gebaseerd op de resultaten van de visuele beoordeling werden de personen naar ernst onderverdeeld in drie groepen. De densitometrische parameters van de groepen werden onderling vergeleken en op correlatie met de visuele beoordelingresultaten onderzocht. Progressie van stoflongen ging op beide inspiratieniveaus gepaard met hogere dichtheidswaarden in zowel boven- als ondervelden. De densitometrische parameters van de groepen verschilden alleen significant op $90 \%$ van de vitalle capaciteit. CT longdensitometrie correleerde goed met de visuele score, wederom alleen op $90 \%$ van de vitale capaciteit. CT sneden van de bovenvelden van de long zijn het meest geschikt ter vaststelling van stoflongen.

\section{Conclusie}

De huidige CT scanners zijn, mits goed gekalibreerd, uitermate geschikt voor densitometrische studies van de long. Echter, om algemeen vergelijkbare resultaten te verkrijgen is het van groot belang eenzelfde scan-en analyse protocol te hanteren. Voor densitometrisch onderzoek van de long op intermediaire inspiratieniveaus is spirometrische controle een vereiste. CT densitometrie kan nuttig zijn voor de diagnostisering, kwantificatie en het vervolgen van emfyseem en interstitiele longziekten.

\section{Toekomstige ontwikkelingen}

De ontwikkeling van snelle volumescanning maakt het mogelijk gedurende én periode van ademinhouden de gehele thorax te scannen. Gebruik makend van deze techniek kan men het longvolume en de longmassa bepalen met een nauwkeurigheid die veel groter is dan in het verleden haalbaar was. Met geschikte software voor de analyse van de zo verkregen omvangrijke datasets kan volumescanning wellicht van nut zijn in het kader van volumereductiechirurgie en longitudinale studies van verscheidene vormen van longziekten.

Resectie van niet functionerende gebieden van longweefsel - volumereductiechirurgie - is inmiddels uitgegroeid tot een veelbelovende therapie voor een kleine groep van geselecteerde patiënten met ernstig invaliderend emfyseem. Volumetrische CT densitometrie kan behulpzaam zijn bij het selecteren van kandidaten clie van deze behandeling kunnen profiteren. Voor de chirurg kan objectieve vaststelling van de locatie en de ernst van emfyseem nuttig zijn zodat hij het meest emfysemateuze longweefsel kan reseceren. Samen met klinische bevindingen en longfunctie-onder- 
zoek kunnen densitometrische data mogelijk helpen inzicht te verschaffen in de mechanismen die verantwoordelijk zijn voor verbetering na behandeling en langere termijn ontwikkeling van de aandoening.

Longitudinale studies van longmassa kunnen erg de moeite waard zijn in combinatie met metabole studies. Er is groeiende interesse in de relatie tussen de voedingsstatus en longarchitectuur. Langdurige ondervoeding en emfyseem veranderen de structuur van de luchthoudende ruimten distaal van de bronchiolus terminalis op min of meer overeenkomstige wijze. Dierexperimenteel onderzoek heeft herhaaldelijk aangetoond dat het gewicht van de long tijdens langdurig vasten vermindert. Een groot aantal patiënten dat lijdt aan COPD ondergaat een geleidelijk maar significant gewichtsverlies gedurende het beloop van hun ziekte. Het potentiële effect op het longgewicht is onbekend. De in vivo bepaling van de longdichtheid is dan interessant in het kader van de begripsvorming omtrent de pathofysiologie van door vasten geïnduceerde veranderingen in de pulmonale architectuur, alsook om de effectiviteit van therapeutische strategieën ter verbetering van de voedingstatus te evalueren. 


\section{Acknowledgments}

I feel greatly indebted to many persons who have contributed to the realization of this thesis.

Review of the manuscript: Prof dr J.M.A. van Engelshoven

Dr G.J. Kemerink

Prof dr E.F.M. Wouters

CT scan acquisitions: $\quad$ G.G.H. Wijnhoven

Secretarial help: $\quad$ H.M.J.C. Kengen

Statistical help: $\quad$ Drs A.G.H. Kessels

Software development: Dr G.J. Kemerink, B.J. Pellis, Ir G.R.P. Thelissen

Data analysis: Drs H.H. Kruize

Correction and translation: Dr G.J. Kemerink, Prof dr ग.T. Wilmink

Coordination of patients: Drs M.P.K.J. Engelen, H.M.J.C. Kengen, Dr R.P.F. Schins

Referral of patients: $\quad$ Drs I.D. Does, Drs R. Mostert 



\section{Curriculum vitae}

Rob J.S. Lamers

1958 Geboren te Leiden

1978 Eindexamen Atheneum- $\beta$

Coriovallum College Heerien

1979

Studie geneeskunde

Rijks Universitair Centrum Antwerpen

1980-1986 Studie geneeskunde

Universiteit van Utrecht

1986-1991 Opleiding radiologie

Academisch Ziekenhuis Maastricht

Opleider: Prof dr B.K. Janevski

$1991-$

Werkzaam alls radioloog

Academisch Ziekenhuis Maastricht

Hoofd: Prof dr J.M.A. van Engelshoven 